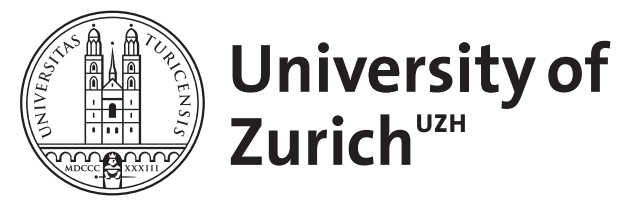

\title{
Relative torsion
}

\author{
Burghelea, D ; Friedlander, L ; Kappeler, T
}

\begin{abstract}
This paper achieves, among other things, the following: - It frees the main result of [9] from the hypothesis of determinant class and extends this result from unitary to arbitrary representations. • It extends (and at the same times provides a new proof of) the main result of Bismut and Zhang [3] from finite dimensional representations of $\Gamma$ to representations on an A-Hilbert module of finite type (A a finite von Neumann algebra). The result of [3] corresponds to $\mathrm{A}=\mathrm{C}$. • It provides interesting real valued functions on the space of representations of the fundamental group $\Gamma$ of a closed manifold $\mathrm{M}$. These functions might be a useful source of topological and geometric invariants of M. These objectives are achieved with the help of the relative torsion, first introduced by Carey, Mathai and Mishchenko [12] in special cases. The main result of this paper calculates explicitly this relative torsion (cf. Theorem $1.1)$.
\end{abstract}

DOI: https://doi.org/10.1142/S0219199701000287

Posted at the Zurich Open Repository and Archive, University of Zurich

ZORA URL: https://doi.org/10.5167/uzh-22004

Journal Article

Originally published at:

Burghelea, D; Friedlander, L; Kappeler, T (2001). Relative torsion. Communications in Contemporary Mathematics, 3(1):15-85.

DOI: https://doi.org/10.1142/S0219199701000287 


\section{Relative torsion}

\section{Burghelea (Ohio State University) \\ L. Friedlander (University of Arizona) \\ T. Kappeler (University of Zürich)}

June 17, 1999

Abstract: This paper achieves, among other things, the following:

- It frees the main result of [BFKM] from the hypothesis of determinant class and extends this result from unitary to arbitrary representations.

- It extends (and at the same times provides a new proof of) the main result of Bismut and Zhang [BZ] from finite dimensional representations of $\Gamma$ to representations on an $\mathcal{A}$-Hilbert module of finite type ( $\mathcal{A}$ a finite von Neumann algebra). The result of [BZ] corresponds to $\mathcal{A}=\mathbb{C}$.

- It provides interesting real valued functions on the space of representations of the fundamental group $\Gamma$ of a closed manifold $M$. These functions might be a useful source of topological and geometric invariants of $M$.

These objectives are achieved with the help of the relative torsion $\mathcal{R}$, first introduced by Carey, Mathai and Mishchenko [CMM] in special cases. The main result of this paper calculates explicitly this relative torsion (cf Theorem 0.1). 


\section{Table of contents}

0 . Introduction

0.1 Summary of the results

0.2 Definitions and outline of the paper

1. Operators and complexes

\subsection{Operators}

1.2 Complexes

2. Relative torsion and its Witten deformation

2.1 Relative torsion

2.2 Witten deformation of the relative torsion

3. Proof of Proposition 0.1 in the case $g=g^{\prime}$

3.1 Outline of the proof

3.2 Asymptotic expansion of $\log \mathcal{R}_{s m}(t)$

3.3 Asymptotic expansion of $\log T_{l a}(t)$

4. Anomalies of the relative torsion

4.1 Metric and Hermitian anomalies

4.2 Proof of Proposition 0.1

4.3 Anomaly with respect to triangulation

5. Proof of Theorem 0.1

5.1 Proof of Theorem 0.1

5.2 An invariant for odd dimensional manifolds.

Appendix A Lemma of Carey-Mathai-Mishchenko

Appendix B Determinant class property

\section{Introduction}

\subsection{Summary of the results}

Let $\left(M, x_{0}\right)$ be a closed, connected manifold with base point $x_{0}$ and $\rho$ : $\Gamma \rightarrow G l_{\mathcal{A}}(\mathcal{W})$ a representation of $\Gamma:=\pi_{1}\left(M ; x_{0}\right)$ on an $\mathcal{A}$-Hilbert module $\mathcal{W}$ of finite type with $\mathcal{A}$ a finite von Neumann algebra. Denote by $\mathcal{E} \rightarrow M$ the vector bundle, associated to $M$ and $\rho$, equipped with the canonical flat connection $\nabla$. 
Let $\mu$ be a Hermitian structure of $\mathcal{E}$ and $\tau=\left(h, g^{\prime}\right)$ a (generalized) triangulation of $M$ (cf subsection 0.2).

The relative torsion is a numerical invariant associated to $\mathcal{F}=(M, \rho, \mu, g, \tau)$. Unlike the analytic torsion $T_{a n}$ associated to $(M, \rho, \mu, g)$, or the Reidemeister torsion $T_{\text {Reid }}$ associated to $\mathcal{F}$ (cf subsection 0.2 ), both of which are defined only when $(M, \rho)$ is of determinant class (cf Appendix B), $\mathcal{R}$ is always defined. If $(M, \rho)$ is of determinant class the relative torsion turns out to be the quotient of the analytic and Reidemeister torsion (cf (2.15)). However, as shown in Appendix B, there are many pairs $(M, \rho)$ which are not of determinant class.

Proposition 0.1 Assume $\mu_{0}$ is a Hermitian structure on the bundle induced by $\rho$ which is parallel in the neighborhood of $\mathrm{Cr}(h)$.

(i) $\log \mathcal{R}=\int_{M \backslash C r(h)} \alpha_{\mathcal{F}}$ where $\alpha_{\mathcal{F}}$ is a density on $M \backslash C r(h)$, which vanishes on a neighborhood of $\mathrm{Cr}(h)$ and is a local quantity (cf subsection 0.2), depending on $\rho, \mu, g$, and $X:=-\operatorname{grad}_{g^{\prime}} h$.

(ii) If $\mu$ is parallel then $\mathcal{R}=1$.

Following the work of Bismut Zhang [BZ], we consider the closed one-form $\theta(\rho, \mu) \in \Omega^{1}(M)$, the form $\Psi(T M, g) \in \Omega^{n-1}\left(T M \backslash M ; \mathcal{O}_{T M}\right)\left(\mathcal{O}_{T M}\right.$ the orientation bundle of $T M)$, and for two Hermitian structures $\mu_{1}$ and $\mu_{2}$ on the bundle $\mathcal{E} \rightarrow M$ induced from $\rho$, the smooth function $V\left(\rho, \mu_{1}, \mu_{2}\right) \in \Omega^{0}(M)$ (cf section 4). When $\mu$ is parallel with respect to $\nabla$ on an open set $U, U \subset M$, then $\theta(\rho, \mu)=0$ on $U$. Denote by $e(M, g)$ the Euler form associated with the Riemannian metric $g$. The triangulation $\tau=\left(h, g^{\prime}\right)$ provides the vector field $X=$ $-\operatorname{grad}_{g^{\prime}} \mathrm{h}$ which will be regarded as a smooth map $X: M \backslash C r(h) \rightarrow T M \backslash M$. The above quantities are used in formulae expressing the change of the relative torsion when one varies the Riemannian metric $g$, the Hermitian structure $\mu$, or the generalized triangulation $\tau$ (cf Propositions 4.1-4.3). These formulae are used to derive the following

Theorem 0.1 Assume $\mu_{0}$ is a Hermitian structure on the bundle induced by $\rho$ which is parallel in the neighborhood of $\mathrm{Cr}(h)$ and $\mu$ is an arbitrary Hermitian structure. Then $\mathcal{R}=\mathcal{R}(M, \rho, \mu, g, \tau)$ is given by

$$
\begin{gathered}
\log \mathcal{R}=(-1)^{n+1} \int_{M \backslash C r(h)} \theta\left(\rho, \mu_{0}\right) \wedge X^{*}(\Psi(T M, g))+\int_{M} V\left(\rho, \mu, \mu_{0}\right) e(M, g)- \\
-\sum_{x \in C r(h)}(-1)^{\operatorname{ind}(\mathrm{x})} V\left(\rho, \mu, \mu_{0}\right)(x) .
\end{gathered}
$$

In the case $M$ is of odd dimension, the above formula for $\log \mathcal{R}$ simplifies,

$$
\log \mathcal{R}=\int_{M \backslash C r(h)} \theta\left(\rho, \mu_{0}\right) \wedge X^{*}(\Psi(T M, g))-\sum_{x \in C r(h)}(-1)^{\mathrm{ind}(\mathrm{x})} V\left(\rho, \mu, \mu_{0}\right)(x) .
$$


Since $\mu_{0}$ is parallel in the neighborhood of the critical points the first integral in the formula evaluating $R$ is convergent, and this is the reasons $\mu_{0}$ is necessary. The formulas in Theorem 0.1 have been discovered by Bismut-Zhang in the case $\mathcal{A}=\mathbb{C}$ (we choose a slightly different definition of $\theta(\rho, \mu)$ which is half of BismutZhang's). Theorem 0.1, applied in this case, implies the main result in [BZ], as in this case $\mathcal{R}=T_{a n} / T_{\text {Reid }}$. The proof of Theorem 0.1 provides a conceptually new proof of the formula of [BZ]. In particular Theorem 0.1 also shows that the formula of Bismut-Zhang for the quotient of analytic and Reidemeister torsion remains true for the quotient of $L_{2}-$ analytic and Reidemeister torsions, cf subsection 5.1, Remark to Theorem 0.1. This answers a question, raised by several experts in the field.

In [BFKM], we have shown that for a unitary representations $\rho$ of $\Gamma$ with $(M, \rho)$ of determinant class, $T_{a n}=T_{\text {Reid }}$. Theorem 0.1 extends this result to an arbitrary representation and reformulates this result in a way that it holds without the assumption of $(M, \rho)$ being of determinant class.

Denote by $\operatorname{Rep}(\Gamma ; \mathcal{W})$ the space of representations of $\Gamma$ on $\mathcal{W}$. The group $\Gamma$ is a finitely presented, let us say with generators $\gamma_{1}, \ldots, \gamma_{N_{1}}$ and relations $R_{j}\left(\gamma_{1}, \ldots, \gamma_{N_{1}}\right)=e_{\Gamma}\left(1 \leq j \leq N_{2}\right)$. Hence $\operatorname{Rep}(\Gamma ; \mathcal{W})$ is a level set of the holomorphic map induced by the relations, $G l_{\mathcal{A}}(\mathcal{W})^{N_{1}} \rightarrow \operatorname{Hom}_{\mathcal{A}}(\mathcal{W})^{N_{2}}$ and thus a complex analytic space (of infinite dimension if $\operatorname{dim}_{\mathbb{C}} \mathcal{W}$ is infinite). Using $\mathcal{R}(M, \rho, \mu, g, \tau)$ we will construct in section 5.2 a smooth invariant for a pair of a closed odd dimensional manifold with fundamental group $\Gamma$ and an Euler structure (cf section 5.2 for the definition of Euler structure). This invariant will be a real analytic function on $\operatorname{Rep}(\Gamma ; \mathcal{W})$. It will be shown in a forthcoming paper that this function and other similarly defined functions associated with even dimensional manifolds, are locally the real part of holomorphic functions on $\operatorname{Rep}(\Gamma ; \mathcal{W})$. As an example (cf section 5.2), we will calculate this function in the case $M=N \times S^{1}$ with $N$ a simply connected manifold of even dimension and $E$ the canonical Euler structure on $N \times S^{1}$. In this case, $\operatorname{Rep}(\Gamma ; \mathcal{W}) \simeq G l_{\mathcal{A}}(\mathcal{W})$ and the function is equal to (cf Proposition 5.1) $-\frac{1}{2} \chi(N) \log \operatorname{det}\left(A^{*} A\right)^{1 / 2}$ where $A \in G l_{\mathcal{A}}(\mathcal{W})$, det is the determinant in the sense of Fuglede- Kadison (cf.[BFKM]), and $\chi(N)$ is the Euler-Poincaré characteristic of $N$. This calculation shows that the invariant is nontrivial. In [BFK 5] we extend the results of this paper to the relative torsion for a homotopy triangulation.

\subsection{Definitions and outline of the paper}

Let $\left(M, x_{0}\right)$ be a closed manifold with base point $x_{0}, \rho: \Gamma \rightarrow G l_{\mathcal{A}}(\mathcal{W})$ a representation of $\Gamma:=\pi_{1}\left(M ; x_{0}\right)$ on an $\mathcal{A}$-Hilbert module $\mathcal{W}, \mathcal{E} \rightarrow M$ the vector bundle associated to $\rho$, equipped with the canonical flat connection $\nabla$, and $\mu$ a Hermitian structure of $\mathcal{E}$.

deRham complex: Denote by $(\Omega(M ; \mathcal{E}), d)$ the deRham complex of smooth forms 
with values in $\mathcal{E}$. Here $d_{k}: \Omega^{k} \rightarrow \Omega^{k+1}$ is the exterior differential determined by $\nabla$. A Riemannian metric $g$ on $M$ together with the Hermitian structure $\mu$ on $\mathcal{E}$ allow to introduce an inner product on $\Omega^{k}$ and therefore determine an adjoint $d_{k}^{*}$ of $d_{k}$. Denote by $\Delta_{k}=d_{k}^{*} d_{k}+d_{k-1} d_{k-1}^{*}$ the Laplacian on $k$-forms. These Laplacians can be used to introduce $s-$ Sobolev norms on $\Omega$. We denote by $H_{s}\left(\Lambda^{k}(M ; \mathcal{E})\right)$ the completion of $\Omega^{k}$ with respect to the s-Sobolev norm. This leads to a family of complexes $H_{s}(\Lambda(M ; \mathcal{E}))$ of $\mathcal{A}$-Hilbert modules.

Analytic torsion $T_{a n}$ : The Laplacians $\Delta_{k}$ admit a regularized determinant $\operatorname{det} \Delta_{k}$ in the von Neumann sense. If $(M, \rho)$ is of determinant class (cf Appendix B), these determinants do not vanish so that the analytic torsion can be defined by

$$
\log T_{a n}(M, \rho, g, \mu):=1 / 2 \sum_{q}(-1)^{q+1} q \log \operatorname{det} \Delta_{q} .
$$

(The concept of determinant class was introduced in [BFKM] Definition 4.1 and will be reviewed in Appendix B.)

Combinatorial complex: A generalized triangulation of $M$ is a pair $\tau=\left(h, g^{\prime}\right)$ with the following properties:

(T1) $\quad h: M \rightarrow \mathbb{R}$ is a smooth Morse function; ${ }^{1}$

(T2) $g^{\prime}$ is a Riemannian metric so that $-\operatorname{grad}_{g^{\prime}} h$ satisfies the Morse Smale condition (for any two distinct critical points $x$ and $y$ of $h$, the stable manifold $W_{x}^{+}$and the unstable manifold $W_{y}^{-}$, with respect to $-\operatorname{grad}_{g^{\prime}} h$, intersect transversely);

(T3) in a neighborhood of any critical point of h one can introduce local coordinates such that, with $q$ denoting the index of this critical point,

$$
h(x)=h(0)-\left(x_{1}^{2}+\ldots+x_{q}^{2}\right) / 2+\left(x_{q+1}^{2}+\ldots+x_{d}^{2}\right) / 2
$$

and the metric $g^{\prime}$ is Euclidean in these coordinates.

A generalized triangulation $\tau$ and a Let $p: \tilde{M} \rightarrow M$ be the universal covering of $M$ and denote by $\tilde{g}$ and $\tilde{\tau}=\left(\tilde{h}, \tilde{g}^{\prime}\right)$ the lifts of $g$ and $\tau$ on $\tilde{M}$. Denote by $C r_{q}(\tilde{h}) \subset \tilde{M}$, resp. $C r_{q}(h) \subset M$, the set of critical points of index $q$ of $\tilde{h}$, resp. $h$, and let $\operatorname{Cr}(\tilde{h})=\cup_{q} C r_{q}(\tilde{h})$. Notice that the group $\Gamma$ acts freely on $C r_{q}(\tilde{h})$ and the quotient set can be identified with $C r_{q}(h)$.

An orientation

$$
\mathcal{O}_{h}:=\left\{\mathcal{O}_{\tilde{x}} \mid \tilde{x} \in \operatorname{Cr}(\tilde{h})\right\}
$$

is provided by a collection of orientations $\mathcal{O}_{\tilde{x}}$ for the unstable manifolds $W_{\tilde{x}}^{-}$ that are $\Gamma$-invariant.

\footnotetext{
${ }^{1}$ In $[\mathrm{BFKM}]$, the Morse function $\mathrm{h}$ was supposed to be selfindexing $(h(x)=\operatorname{index}(x)$ for any critical point $x$ of $h$ ). For our proof of Theorem 0.1 (step 2), it is convenient to drop this condition (cf [BFK2]).
} 
To the system $\left(M, \rho, \mu, \tau, \mathcal{O}_{h},\right)$, we associate a cochain complex of finite type over the von Neumann algebra $\mathcal{A}, \mathcal{C} \equiv \mathcal{C}\left(M, \rho, \mu, \tau, \mathcal{O}_{h}\right)=\left\{\mathcal{C}^{q}, \delta_{q}\right\}$, where $\mathcal{C}^{q}=\oplus_{x \in C r_{q}(h)} \mathcal{E}_{x}$, which can be identified with the module of $\Gamma$-equivariant maps $f: C r_{q}(\tilde{h}) \rightarrow \mathcal{W}$, and the maps $\delta_{q}: \mathcal{C}^{q} \rightarrow \mathcal{C}^{q+1}$ are given by

$$
\delta_{q}(f)(\tilde{x}):=\sum_{\tilde{y} \in C r_{q}(\tilde{h})} \nu_{q+1}(\tilde{x}, \tilde{y}) f(\tilde{y})
$$

where $\nu_{q}: C r_{q}(\tilde{h}) \times C r_{q-1}(\tilde{h}) \rightarrow \mathbb{Z}$ is defined by $\nu_{q}(\tilde{x}, \tilde{y}):=$ intersection number $\left(W_{\tilde{x}}^{-} \cap V, W_{\tilde{y}}^{+} \cap V\right)$ with $V:=\tilde{h}^{-1}\left(q-\frac{1}{2}\right)$. The cochain complex $\mathcal{C}\left(M, \rho, \mu, \tau, \mathcal{O}_{h}\right)$ depends on $\mu$ only via $\mu_{x}, x \in C r(h)$. Let $\Delta_{q}^{c o m b}:=\delta_{q}^{*} \delta_{q}+\delta_{q-1} \delta_{q-1}^{*}$ denote the combinatorial Laplacian.

Reidemeister torsion $T_{\text {Reid }}: \Delta_{q}^{c o m b}$ admits a regularized determinant $\operatorname{det} \Delta_{q}^{c o m b}$ in the von Neumann sense which, under the additional hypothesis of $(M, \rho)$ being of determinant class (cf Appendix B), does not vanish. Under this hypothesis, the combinatorial torsion can be defined

$$
\log T_{c o m b}(M, \rho, \mu, \tau):=1 / 2 \sum_{q}(-1)^{q+1} q \log \operatorname{det} \Delta_{q}^{c o m b} .
$$

It can be shown that the combinatorial torsion is independent of the choice of $\mathcal{O}_{h}$. Given a Riemannian metric $g$, the metric part $T_{m e t}$ of the Reidemeister torsion is defined as in $[\mathrm{BFKM}]$ and the Reidemeister torsion $T_{\text {Reid }}$ is then given by

$$
\log T_{\text {Reid }}:=\log T_{\text {comb }}+\log T_{\text {met }}
$$

Relative torsion: Notice that the integration on the $q$-cells of the generalized triangulation $\tau$ which are given by the unstable manifolds of $-\operatorname{grad}_{g^{\prime}} h$, defines a morphism

$$
\text { Int }:(\Omega, d) \rightarrow(\mathcal{C}, \delta),
$$

i.e. $\operatorname{Int}_{q}: \Omega^{q} \rightarrow \mathcal{C}^{q}$ is an $\mathcal{A}$-linear map so that $\delta_{q} \operatorname{Int}_{q}=\operatorname{Int}_{q+1} d_{q}$ (cf Appendix by $\mathrm{F}$. Laudenbach in [BZ] or [B1]). We would like to define the relative torsion of the system $(M, \rho, \mu, g, \tau)$ as the torsion of the mapping cone defined by the integration map. Unfortunately, the torsion of this mapping cone cannot be defined (at least not in an obvious way); a first difficulty comes from the fact that the integration maps $\operatorname{Int}_{k}$ do not extend to closed maps, defined on a dense domain of $H_{0}\left(\Lambda^{k}(M ; \mathcal{E})\right)$. However, for $s$ sufficiently large, the integration morphism has an extension Int $_{s}$

$$
\text { Int }_{s}: H_{s}(\Lambda(M ; \mathcal{E})) \rightarrow \mathcal{C}
$$


to a bounded morphism. We then consider the composition $g_{s}$

$$
g_{s}: H_{0}(\Lambda(M ; \mathcal{E})) \stackrel{(\Delta+I d)}{\longrightarrow}{ }^{-s / 2} H_{s}(\Lambda(M ; \mathcal{E})) \stackrel{I n t_{s}}{\longrightarrow} \mathcal{C}
$$

and prove that $g_{s}$ induces an isomorphism in algebraic cohomology $\operatorname{Ker}\left(d_{k}\right) /$ range $\left(d_{k-1}\right)$ as well as in reduced cohomology (cf Proposition 2.5)

$$
\operatorname { K e r } ( d _ { k } ) \longdiv { \text { range } ( d _ { k - 1 } ) } \text {. }
$$

This implies that the mapping cone $\mathcal{C}\left(g_{s}\right)$, defined by

$$
\mathcal{C}\left(g_{s}\right)_{k}:=\mathcal{C}^{k-1} \oplus H_{0}\left(\Lambda^{k}(M ; \mathcal{E})\right) ; d\left(g_{s}\right)_{k}:=\left(\begin{array}{cc}
-\delta_{k-1} & g_{k ; s} \\
0 & d_{k}
\end{array}\right),
$$

is an algebraically acyclic cochain complex (i.e. $\operatorname{Kerd}\left(g_{s}\right)_{k} /$ range $\left(d\left(g_{s}\right)_{k-1}\right)=$ 0 ). In particular the complex $\mathcal{C}\left(g_{s}\right)$ is a cochain complex of $\mathcal{A}$-Hilbert modules (cf Lemma 1.11) whose Laplacians $\Delta\left(g_{s}\right)_{k}$ are unbounded operators which admit a nonvanishing regularized determinant $\operatorname{det} \Delta\left(g_{s}\right)_{k}$ in the von Neumann sense. One of the purposes of section 1 is to establish this result.

The relative torsion $\mathcal{R}_{s}$ is defined as the torsion $T\left(\mathcal{C}\left(g_{s}\right)\right)$ of the mapping cone $\mathcal{C}\left(g_{s}\right)$,

$$
\log \mathcal{R}_{s}:=\log T\left(\mathcal{C}\left(g_{s}\right)\right):=\frac{1}{2} \sum_{k}(-1)^{k+1} k \log \operatorname{det}\left(\Delta\left(g_{s}\right)_{k}\right) .
$$

In section 2 , we show that $\log \mathcal{R}_{s}$ is independent of $s$ ( $s$ sufficiently large) and thus provide a well defined number which we denote by $\log \mathcal{R}$.

As first shown in $[\mathrm{CMM}]$ in a slightly different and less general version, one verifies that if $(M, \rho)$ is of determinant class, then $\log \mathcal{R}=\log T_{a n}-\log T_{\text {Reid }}$ (cf (2.15)) where $T_{a n}$, resp. $T_{\text {Reid }}$, denotes the analytic torsion resp. Reidemeister torsion. The main result of [BFKM] can thus be stated as follows: When $(M, \rho)$ is of determinant class and $\mu$ is parallel then $\mathcal{R}=1$.

Representations: Let $\rho: \Gamma \rightarrow G l_{\mathcal{A}}(\mathcal{W})$ be a representation of $\Gamma$ on a $\mathcal{A}$-Hilbert module of finite type. The representation $\rho^{\sharp}: \Gamma \rightarrow G l_{\mathcal{A}}(\mathcal{W}) d u a l$ to $\rho$ is defined by $\rho^{\sharp}(\gamma):=\rho\left(\gamma^{-1}\right)^{*}(\gamma \in \Gamma)$ where ${ }^{*}$ denotes the adjoint in $G l_{\mathcal{A}}(\mathcal{W})$ with respect to the inner product of $\mathcal{W}$. The representation $\rho$ is said to be unitary if $\rho^{\sharp}=\rho$. Notice that the flat bundle $\mathcal{E} \rightarrow M$ induced by a unitary representation admits a parallel Hermitian structure.

A representation $\rho^{\prime}: \Gamma \rightarrow G l_{\mathcal{A}^{\prime}}\left(\mathcal{W}^{\prime}\right)\left(\mathcal{W}^{\prime}\right.$ a $\mathcal{A}^{\prime}$-Hilbert module of finite type) is said to be isomorphic to $\rho$ if there exist an isomorphism $\theta: \mathcal{A} \rightarrow \mathcal{A}^{\prime}$ ( with $\left.\operatorname{tr}_{\mathcal{A}^{\prime}}(\theta a)=\operatorname{tr}_{\mathcal{A}}(a), \forall a \in \mathcal{A}\right)$ and an isomorphism $\Theta: \mathcal{W} \rightarrow \mathcal{W}^{\prime}$, such that $\Theta$ is $\theta$-linear. As an example, we mention that $\rho \otimes \rho^{\sharp}$ and $\rho^{\sharp} \otimes \rho$ are isomorphic. 
We denote by $\operatorname{Rep}(\Gamma, \mathcal{W})$ the complex analytic space of representations (cf subsection 0.1).

Densities and local quantities: A density $\alpha$ on a manifold $M$ of dimension $n$ is a smooth $n$-form with coefficients in the orientation bundle $\mathcal{O}_{M} \rightarrow M$. Densities can be integrated on relative compact open subsets $U \subset M$, and the integral is denoted by $\int_{U} \alpha$.

In this paper, we use the word local quantity for an assignment which to any set of data $\mathcal{G}:=(\mathcal{E} \rightarrow M, \nabla, \mu, g, X)$ consisting of a bundle $\mathcal{E} \rightarrow M$, a flat connection $\nabla$, a Hermitian structure $\mu$, a Riemannian metric $g$, and a nonzero vectorfield $X$ on $M$, provides a density $\alpha(\mathcal{G})$ with the following properties:

(P1) For any open set $U \subset M, \alpha\left(\left.\mathcal{G}\right|_{U}\right)=\left.\alpha(\mathcal{G})\right|_{U}$.

(P2) If $(\phi, \Phi)$ is an isomorphism between $\mathcal{G}_{1}$ and $\mathcal{G}_{2}$ (i.e. $\Phi: \mathcal{E}_{1} \rightarrow \mathcal{E}_{2}$ is a bundle isomorphism above the diffeomorphism $\phi: M_{1} \rightarrow M_{2}$ such that $\left.\phi^{*} \nabla_{2}=\nabla_{1}, \phi^{*} \mu_{2}=\mu_{1}, \phi^{*} g_{2}=g_{1}, \phi^{*} X_{2}=X_{1}\right)$ then $\phi^{*} \alpha\left(\mathcal{G}_{2}\right)=\alpha\left(\mathcal{G}_{1}\right)$.

To a system $\mathcal{F}=(M, \rho, \mu, g, \tau)$ we associate the set of data $\mathcal{G}_{\mathcal{F}}=\left(\left.\mathcal{E}\right|_{M \backslash C r(h)} \rightarrow M \backslash C r(h), \nabla, \mu, X\right)$ where $(\mathcal{E} \rightarrow M, \nabla)$ is the bundle with flat connection $\nabla$, canonically associated to $\rho$ and $X=-$ grad $_{g^{\prime}} h$.

Let us briefly comment on the proofs of the results stated in subsection 0.1: The proof of Proposition 0.1 (cf section 3 and subsection 4.2) uses the Witten deformation of the deRham complex, given by the differentials $d_{k}(t):=e^{-t h} d_{k} e^{t h}$. We consider a deformation $\mathcal{R}(t)$ of the relative torsion $\mathcal{R}=\mathcal{R}(0)$, induced by the Witten deformation of the deRham complex. From many different possibilities for defining the deformation $\mathcal{R}(t)$ we choose one for which the variation $\frac{d}{d t} \log \mathcal{R}(t)$ can be computed. We define $\mathcal{R}(t)$ as the torsion of the mapping cone associated to

$$
\left(H_{0}(\Lambda(M ; \mathcal{E})), d(t)\right) \stackrel{e^{t h}}{\rightarrow}\left(H_{0}(\Lambda(M ; \mathcal{E})), d\right) \stackrel{g_{\S}}{\rightarrow}(\mathcal{C}, \delta),
$$

which will be checked to be independent of $s$, cf Proposition 2.7 and Definition 2.8. The variation of $\log \mathcal{R}(t)$ can be computed to be (cf Theorem 2.1)

$$
\frac{d}{d t} \log \mathcal{R}(t)=\operatorname{dim} \mathcal{E}_{x} \cdot \int_{M} h e(M, g),
$$

where $e(M, g)$ is the Euler form of the tangent bundle equipped with the LeviCività connection induced from $g$. It follows that $\log \mathcal{R}(t)$ is given by $\log \mathcal{R}+$ $t \int_{M} h e(M, g)$.

To continue our discussion we say that a function $G: \mathbb{R} \rightarrow \mathbb{R}$ admits an asymptotic expansion for $t \rightarrow \infty$ if there exist a sequence $i_{1}>i_{2}>\ldots>i_{N}=0$ and constants $\left(a_{k}\right)_{1 \leq k \leq N},\left(b_{k}\right)_{1 \leq k \leq N}$ such that, as $t \rightarrow \infty$, 


$$
G(t)=\sum_{1}^{N} a_{k} t^{i_{k}}+\sum_{1}^{N} b_{k} t^{i_{k}} \log t+o(1) .
$$

For convenience, we denote by $F T(G(t))$ or $F T_{t=\infty}(G)$ the coefficient $a_{N}$ in the asymptotic expansion of $G(t)$ and refer to it as the free term of the expansion. Notice that the equality $(0.1)$ implies that $\log \mathcal{R}(t)$ admits an asymptotic expansion of the form (0.2) with $\log \mathcal{R}$ as the free term.

By different considerations outlined below, we derive the existence of an asymptotic expansion for $\log \mathcal{R}(t)$ of the form (0.2) and calculate the free term of this expansion as the integral on $M \backslash C r(h)$ of a local density expressed in terms of $g, \mu$ and $\tau$. The proof of Proposition 0.1 will then be derived from the comparison of these two calculations. Using Witten deformation we decompose $\log \mathcal{R}(t)$ into two parts

$$
\log \mathcal{R}(t)=\log \mathcal{R}_{s m}(t)+\log T_{l a}(t),
$$

and show that each of the two parts admit an asymptotic expansion of the form (0.2) whose free terms can be computed. Precisely, we consider the Witten deformation $(\Omega(M ; \mathcal{E}), d(t))$ of the deRham complex, $d(t)=e^{-t h} d e^{t h}$. For $t$ sufficiently large, the deformed deRham complex can be decomposed,

$$
(\Omega(M, \mathcal{E}), d(t))=\left(\Omega_{s m}(t), d(t)\right) \oplus\left(\Omega_{l a}(t), d(t)\right),
$$

corresponding to the small and the large part of the spectrum of the deformed Laplacians $\Delta_{k}(t)$. This decomposition induces the decomposition

$$
\left(H_{0}(\Lambda(M ; \mathcal{E})), d(t)\right)=\left(\Omega_{s m}(t), d(t)\right) \oplus\left(H_{0, l a}(t), d(t)\right) .
$$

The complex $\left(\Omega_{s m}(t), d(t)\right)$ is of finite type. Denote by $g_{s, s m}(t)$ the restriction of $g_{s} \cdot e^{t h}$ to $\Omega_{s m}(t)$, where $e^{t h}: \Omega(M, \mathcal{E}) \rightarrow \Omega(M, \mathcal{E})$ denotes the multiplication by $e^{t h}$. The mapping cone $\mathcal{C}\left(g_{s, s m}(t)\right)$ is a cochain complex of $\mathcal{A}$-Hilbert modules of finite type which is algebraically acyclic and has a well defined torsion denoted by $\mathcal{R}_{s m}(t)$. As in [BFKM, section 6], one verifies that $\left(H_{0, l a}(t), d(t)\right)$ is algebraically acyclic and has a well defined torsion, denoted by $T_{l a}(t)$. We prove that $\log \mathcal{R}(t)=\log \mathcal{R}_{s m}(t)+\log T_{l a}(t)$ (cf subsection 3.1, statement $(B)$ ).

To prove that $\log \mathcal{R}_{s m}(t)$ has an asymptotic expansion, we show that $\mathcal{R}_{s m}(t)=$ $T(\mathcal{C}(f(t)))$ (cf Proposition 3.1), the torsion of the mapping cone induced by

$$
f(t):\left(\Omega_{s m}(t), d(t)\right) \stackrel{e^{t h}}{\rightarrow}(\Omega, d) \stackrel{\text { Int }}{\rightarrow}(\mathcal{C}, \delta)
$$

and use the Witten-Helffer-Sjöstrand theory as presented in [BFKM] to prove that $\log T(\mathcal{C}(f(t)))$ admits an asymptotic expansion of the type $(0.2)$ and to compute its free term (Proposition 3.1). To analyze $\log T_{l a}(t)$, we proceed as in [BFK1] or [BFKM]. We derive the existence of an asymptotic expansion for $\log T_{l a}(t)$ and a closed formula for its free term from Proposition 3.2 iii). 
This result states the existence of an asymptotic expansion for the difference $\log T_{l a}(t)-\log \tilde{T}_{l a}(t)$ where $(M, \rho, \mu, g, \tau)$ and $(\tilde{M}, \tilde{\rho}, \tilde{\mu}, \tilde{g}, \tilde{\tau})$ are two systems whose Morse functions $h$ and $\tilde{h}$ have the same number of critical points in each index, and provides a formula for its free term.

The proof of Theorem 0.1 ( cf subsection 5.1) uses the anomalies of the relative torsion (Propositions 4.1- 4.3) and Proposition 0.1. The locality of the density given Proposition 0.1 is essential for this proof.

The paper is organized in 4 sections and two appendices.

Section 1: In subsection 1.1 we single out a class of unbounded $\mathcal{A}$-linear operators on $\mathcal{A}$-Hilbert modules ( $\mathcal{A}$ is a von Neumann algebra with finite trace ) for which the regularized determinant can be defined and in subsection 1.2 a class of complexes of $\mathcal{A}$-Hilbert modules for which the Laplacians are operators in the above class. Therefore the torsion of an (algebraically) acyclic complex of such type can be defined. These abstract results are needed because the mapping cone of (a regularized version of) the integration map between the deRham complex and the combinatorial complex has as its components direct sums of $\mathcal{A}$-Hilbert modules of finite type and completions (with respect to a Sobolev norm) of spaces of smooth sections in smooth bundles of $\mathcal{A}$-Hilbert modules of finite type (cf. $[\mathrm{BFKM}]$ for definitions). We show that the mapping cone of a regularized version of the integration map is a complex of the type introduced in subsection 1.2.

Section 2: Using the results of section 1, we introduce in subsection 2.1 the notion of relative torsion $\mathcal{R}$ and in subsection 2.2 the Witten deformation $\mathcal{R}(t)$ of the relative torsion $\mathcal{R}$ and calculate its variation.

Section 3: Proof of Proposition 0.1 in the case $g=g^{\prime}$.

Section 4: We investigate how the relative torsion $\mathcal{R}(M, \rho, \mu, g, \tau)$ varies with respect to the Riemannian metric $g$, the Hermitian structure $\mu$ and the triangulation $\tau$. Further we prove Proposition 0.1 in full generality.

Section 5: Proof of Theorem 0.1 and the construction of an invariant for odd dimensional manifolds.

Appendix A: For the convenience of the reader we present a proof of a slightly stronger version of a Lemma due to Carey-Mathai-Mishchenko.

Appendix B: We review the concept of determinant class and provide a simple example of a pair $(M, \rho), M:=S^{1}$ and $\rho$ a representation of $\pi_{1}\left(S^{1}\right)=\mathbb{Z}$ on $l_{2}(\mathbb{Z})$, which is not of determinant class. The existence of such pairs motivates in part the concept of relative torsion.

Throughout the paper the notions of trace (denoted by Tr), dimension, determinant etc. are always understood in the von Neumann sense. 


\section{Operators and complexes}

\section{$1.1 \quad$ Operators}

Let $\mathcal{W}_{1}, \mathcal{W}_{2}$ be $\mathcal{A}$-Hilbert modules and $\varphi: \mathcal{W}_{1} \rightarrow \mathcal{W}_{2}$ an operator. Denote its domain by $\operatorname{domain}(\varphi) \subseteq \mathcal{W}_{1}$ and its range by $\operatorname{range}(\varphi)=\varphi(\operatorname{domain}(\varphi)) \subseteq \mathcal{W}_{2}$.

Introduce the following properties of $\varphi$.

$O p(1) \quad \varphi$ is $\mathcal{A}$-linear.

$O p(2) \quad \varphi$ is densely defined, i.e. $\overline{\operatorname{domain}(\varphi)}=\mathcal{W}_{1}$.

$O p(3) \quad \varphi$ is closed (i.e. the graph $\Gamma(\varphi)$ of $\varphi$ is closed in $\mathcal{W}_{1} \times \mathcal{W}_{2}$ ).

If, instead of $O p(3), \varphi$ satisfies

Op(3)' $\quad \varphi$ is closable

we consider the minimal extension of $\varphi$ and denote it again by $\varphi$.

In the sequel, we will not distinguish between property $O p(3)$ and $O p(3)^{\prime}$.

An operator $\varphi$ satisfying $O p(1)-O p(3)$, admits an adjoint, $\varphi^{*}$ (cf. e.g. [RS, p 316]) and we can consider the nonnegative, selfadjoint operator $\varphi^{*} \varphi$. Functional calculus permits to define the square root $|\varphi|:=\left(\varphi^{*} \varphi\right)^{1 / 2}$. Then, for any $0<t<$ $\infty$, the heat evolution operator $e^{-t|\varphi|}$ is bounded, nonnegative and selfadjoint. The operators $|\varphi|$ and $e^{-t|\varphi|}$ satisfy the properties $O p(1)-O p(3)$. In the sequel, if not mentioned otherwise, we assume that the operators considered satisfy $O p(1)-O p(3)$.

Denote by $d P(\lambda) \equiv d P_{|\varphi|}(\lambda)$ the (operator valued) spectral measure associated to $|\varphi|$ defined by the orthogonal projectors $P_{|\varphi|}([0, \lambda])(0 \leq \lambda \leq \infty)$

$$
P_{|\varphi|}([0, \lambda])=\int_{0_{-}}^{\lambda_{+}} d P_{|\varphi|}(\lambda)
$$

Most of the operators $\varphi$ considered in this paper will satisfy the following important property

$\operatorname{Op}(4) \quad \operatorname{Tr} P_{|\varphi|}([a, b])<\infty \quad$ (for any $\left.0<a \leq b<\infty\right)$

where

$$
P_{|\varphi|}([a, b]):=\int_{a_{-}}^{b_{+}} d P_{|\varphi|}(\lambda) .
$$

If $\varphi$ satisfies $O p(4)$ one can define the Stiltjes measure $d F_{|\varphi|}(\lambda)$ on the half line $(0, \infty)$, given by $(0<a<b<\infty)$ 


$$
\int_{a_{-}}^{b_{+}} d F_{|\varphi|}(\lambda)=\operatorname{Tr} P_{|\varphi|}([a, b]) .
$$

The next two properties concern the asymptotic behavior of $\operatorname{Tr} P([a, b])$ for $b \nearrow \infty$ and $a \searrow 0$ :

Op(5) There exists $\alpha \geq 0$ such that

$$
\operatorname{Tr} P_{|\varphi|}([1, \lambda])=0\left(\lambda^{\alpha}\right) \text { for } \lambda \nearrow \infty
$$

and

$O p(6) \quad \operatorname{Tr} P_{|\varphi|}([\lambda, 1])=0(1)$ for $\lambda \searrow 0$.

If $\varphi$ satisfies properties $O p(4)$ and $O p(6)$ one can define the spectral distribution functions, associated to $|\varphi|$,

$$
F_{|\varphi|}^{+}(\lambda)=\operatorname{Tr} P_{|\varphi|}((0, \lambda)) ; F_{|\varphi|}(\lambda):=\operatorname{Tr} P_{|\varphi|}((-\infty, \lambda)) .
$$

The spectral distribution function $F_{|\varphi|}^{+}(\lambda)$ can be described variationally as follows, $(\lambda>0)$

$$
\begin{aligned}
F_{|\varphi|}^{+}(\lambda)= & \sup \{\operatorname{dim} L \mid L \subset \operatorname{domain}(|\varphi|), \perp \operatorname{Ker} \varphi, \text { is an } \mathcal{A} \text {-Hilbert } \\
& \text { submodule with }\||\varphi|(x)\|<\lambda\|x\| \forall x \in L\}
\end{aligned}
$$

(cf e.g. [GS], [BFKM]).

If, in addition, $\varphi$ satisfies also $O p(5)$, one can define the heat trace $\theta_{|\varphi|}(t)$ associated to $\varphi$ (excluding zero modes)

$$
\theta_{|\varphi|}(t):=\int_{0_{+}}^{\infty} e^{-t \lambda} d F_{|\varphi|}^{+}(\lambda)(t>0) .
$$

Using integration by parts in the Stiltjes integral (1.5) one concludes from $O p(5)$ that

$$
\theta_{|\varphi|}(t)=0\left(t^{-\alpha}\right)(t \searrow 0) .
$$

Next, let us introduce the 'partial' zeta function $\zeta_{|\varphi|}^{\mathrm{I}}(s)$ associated to the heat trace $\theta_{|\varphi|}(t)$ and defined for $s \in \mathbb{C}$ with Res $>\alpha$ (with $\alpha$ given by Op(5))

$$
\zeta_{|\varphi|}^{\mathrm{I}}(s):=\frac{1}{\Gamma(s)} \int_{0}^{1} t^{s-1} \theta_{|\varphi|}(t) d t
$$

where $\Gamma(s)$ denotes the gamma function. Notice that $\zeta_{|\varphi|}^{\mathrm{I}}(s)$ is holomorphic in the halfplane Res $>\alpha$. 
The following property is needed to define the notion of regularized determinant:

$O p(7) \quad \zeta_{|\varphi|}^{\mathrm{I}}(s)$ has an analytic continuation at $s=0$.

A sufficient condition for $O p(7)$ to hold is the existence of an asymptotic expansion of $\theta_{|\varphi|}(t)$ near $t=0$ of the form

(Asy) $\theta_{|\varphi|}=\sum_{j=0}^{m-1} a_{j} t^{-\alpha_{j}}+a_{m}+R(t)$ where $m \in \mathbb{Z}_{\geq 0}, 0<\alpha_{m-1}$ $<\ldots<\alpha_{0}, a_{0}, \ldots, a_{m} \in \mathbb{R}$ and where $R(t)$ is a bounded, continuous function with $R(t)=0\left(t^{\rho}\right)$ for some $\rho \in \mathbb{R}_{>0}$.

The fact that (Asy) implies $O p(7)$ follows from the following lemma, using integration by parts.

Lemma 1.1 Let $f:(0,1] \rightarrow \mathbb{R}$ be a continuous function of the form

$$
f(t)=\sum_{j=0}^{m-1} a_{j} t^{-\alpha_{j}}+a_{m}+R(t)
$$

with $a_{0}, \ldots, a_{m}, \alpha_{0}, \ldots, \alpha_{m-1}$ and $R(t)$ as in (Asy).

Then, the holomorphic function $\xi(x):=\frac{1}{\Gamma(s)} \int_{0}^{1} t^{s-1} f(t) d t$, defined for Res $>$ $\alpha_{0}$, has a meromorphic continuation to the half plane Res $>-\rho$ with $\rho>0$ as in (Asy). It has only simple poles, located at $s=\alpha_{j}(0 \leq j \leq m-1)$. In particular, $\xi(s)$ is regular at $s=0$ and $\xi(0)=a_{m}$.

Some of the operators $\varphi$ we will consider have the property that 0 is not in the spectrum, i.e.,

$O p(8) \quad$ There exists $\varepsilon>0$ with the property that $\operatorname{Tr} P_{|\varphi|}([0, \varepsilon])=0$.

If $\varphi$ satisfies the properties $O p(1)-O p(8)$, then

$$
\theta_{|\varphi|}(t)=0\left(e^{-t \varepsilon / 2}\right) \text { for } t \rightarrow \infty .
$$

Thus $\int_{1}^{\infty} t^{s-1} \theta_{|\varphi|}(t) d t$ is an entire function of $s$ and, as a consequence,

$$
\zeta_{|\varphi|}^{\mathrm{II}}(s):=\frac{1}{\Gamma(s)} \int_{1}^{\infty} t^{s-1} \theta_{|\varphi|}(t) d t
$$

is a holomorphic function in $s \in \mathbb{C}$ with

$$
\zeta_{|\varphi|}^{\mathrm{II}}(0)=0
$$


(Actually for $\zeta_{|\varphi|}^{\mathrm{II}}(s)$, no zeta regularization is needed (cf [BFKM], subsection 6.1).)

For operators $\varphi$ satisfying properties $O p(1)-O p(8)$, one can introduce the zeta function $\zeta_{|\varphi|}(s)$

$$
\zeta_{|\varphi|}(s):=\zeta_{|\varphi|}^{\mathrm{I}}(s)+\zeta_{|\varphi|}^{\mathrm{II}}(s)=\frac{1}{\Gamma(s)} \int_{0}^{\infty} t^{s-1} \theta_{|\varphi|}(t) d t
$$

for Res $>\alpha$ which has an analytic continuation at $s=0$. This allows to define the volume of the operator $\varphi, \operatorname{Vol} \varphi$, by

$$
\log \operatorname{Vol}(\varphi):=\log \operatorname{det}|\varphi|:=-\left.\frac{d}{d s}\right|_{s=0} \zeta_{|\varphi|}(s)
$$

Definition 1.2 1. An operator $\varphi: \mathcal{W}_{1} \rightarrow \mathcal{W}_{2}$ is said to be of sF type (strong Fredholm type) if

(i) $\varphi$ satisfies $O p(1)-O p(6)$ and

(ii) $\operatorname{dim}(\operatorname{Ker} \varphi):=\operatorname{Tr} P_{|\varphi|}(\{0\})<\infty$.

2. An operator $\varphi$ is a bounded operator of trace class if

(i) $\varphi$ is bounded (hence satisfies $O p(1)-O p(3)$ );

(ii) $\varphi$ satisfies $O p(4)$;

(iii) $\|\varphi\|_{t r}:=\int_{0_{+}}^{\infty} \lambda d F_{|\varphi|}(\lambda)<\infty$.

3. An operator $\varphi$ is said to be $\zeta$-regular if $\varphi$ satisfies $O p(1)-O p(7)$.

4. An operator $\varphi$ is a bounded operator of finite rank if

(i) $\varphi$ is bounded;

(ii) $\operatorname{dim}(\overline{\operatorname{range}(\varphi)})<\infty$.

(As a consequence, $\varphi$ satisfies $O p(4)-O p(6)$ and (Asy) with $m=0$ and $\rho=1$ and thus, $O p(1)-O p(7)$ hold.)

Notice that an operator $\varphi: \mathcal{W}_{1} \rightarrow \mathcal{W}_{2}$ of $\mathrm{sF}$ type might not be bounded in the case where $\operatorname{dim}_{1}=\infty$.

Proposition 1.3 (cf [Di]) Let $u: \mathcal{W}_{1} \rightarrow \mathcal{W}_{2}$ and $v: \mathcal{W}_{2} \rightarrow \mathcal{W}_{3}$ be bounded operators with $u$, respectively $v$, of trace class. Then the composition vu is a bounded operator of trace class and $\|v u\|_{t r} \leq\|v\|\|u\|_{t r}$, respectively, $\|v u\| \leq$ $\|v\|_{t r}\|u\|$. 
As the proof of Proposition 1.3 is fairly standard, we omit it. The following result will be used in the proof of Proposition 1.5, stated below:

Lemma 1.4 Assume that $\varphi: \mathcal{W} \rightarrow \mathcal{W}$ is a nonnegative, selfadjoint operator (i.e $\varphi=|\varphi|$ ) of sF type. Let $0<a<b$ and assume that $\varepsilon>0$ satisfies $\varepsilon<F_{\varphi}(a)$. Then one can choose a smooth function $f \in C_{0}^{\infty}\left(\mathbb{R}_{>_{0}} ; \mathbb{R}\right)$ such that $f(\varphi)$ is a bounded operator of finite rank (hence $f(\varphi)$ is of trace class and commutes with $\varphi$ ) with the following properties:

(i) $F_{\varphi+f(\varphi)}(\lambda)=0$ for $\lambda<$ a (in particular, $\varphi+f(\varphi)$ is 1-1);

(ii) $F_{\varphi+f(\varphi)}(\lambda) \geq \varepsilon$ for $\lambda \geq a$;

(iii) $F_{\varphi+f(\varphi)}(\lambda)=F_{\varphi}(\lambda)$ for $\lambda \geq b$.

Remark This lemma will be applied in the case when $\varphi$ is a pseudodifferential operator. Then $\varphi$ can be chosen so that $f(\varphi)$ is a smoothing operator.

Proof Let $g:[0, \infty) \rightarrow \mathbb{R}$ be a smooth, increasing function defined on $[0, \infty)$ with $g(\lambda)=a$ for $\lambda \leq a, g(\lambda) \leq \lambda$ for $a \leq \lambda \leq b$ and $g(\lambda)=\lambda$ for $\lambda \geq b$. Let $f(\lambda):=g(\lambda)-\lambda$ and define

$$
f(\varphi):=\int_{0}^{\infty} f(\lambda) d P_{\varphi}(\lambda)
$$

\section{Proposition 1.5}

(A) If $\varphi: \mathcal{W} \rightarrow \mathcal{W}$ is of $s F$ type and $u: \mathcal{W} \rightarrow \mathcal{W}$ is a bounded operator of $s F$ type, then $\varphi+u$ is of $s F$ type.

(B) If, in addition, $\varphi$ and $\varphi+u$ are selfadjoint, nonnegative operators and $u$ is of trace class, then there exists $\varepsilon>0$ so that the difference $\zeta_{\varphi}^{\mathrm{I}}(s)-\zeta_{\varphi+u}^{\mathrm{I}}(s)$, defined for Res $\gg 0$,

$$
\zeta_{\varphi}^{\mathrm{I}}(s)-\zeta_{\varphi+u}^{\mathrm{I}}(s)=\frac{1}{\Gamma(s)} \int_{0}^{1} t^{s-1}\left(\theta_{\varphi}(t)-\theta_{\varphi+u}(t)\right) d t
$$

has an analytic continuation to Res $>-\varepsilon$ and its value at $s=0$ is equal to $\operatorname{dim}(\operatorname{Ker} \varphi)-\operatorname{dim}(\operatorname{Ker}(\varphi+u))$.

Remark If $v$ is a bounded operator and $\varphi$ and $u$ are operators as in Proposition 1.5(B), one can, instead of $\theta_{\varphi}(t)$ and $\theta_{\varphi+v}(t)$, consider the function $\theta_{\varphi, v}(t):=$ $\operatorname{Trve}^{-t \varphi}\left(\operatorname{Id}-P_{\varphi}(\{0\})\right)$ and similarly, $\theta_{\varphi+u, v}(t):=\operatorname{Trve}^{-t(\varphi+u)}\left(\operatorname{Id}-P_{\varphi+u}\right.$ $(\{0\}))$. Notice that for $v=I d, \theta_{\varphi, I d}(t)=\theta_{\varphi}(t)(t>0)$. Both expressions, 
$\zeta_{\varphi, v}^{\mathrm{I}}(s):=\frac{1}{\Gamma(s)} \int_{0}^{1} t^{s-1} \theta_{\varphi, v}(t) d t$ and $\zeta_{\varphi+u, v}^{\mathrm{I}}(s):=\frac{1}{\Gamma(s)} \int_{0}^{1} t^{s-1} \theta_{\varphi+u, v}(t) d t$, define holomorphic functions for Res $\gg 0$ and by the same arguments as in the proof of Proposition 1.5(B) (cf below) their difference is holomorphic for Res $>-\varepsilon$ for some $\varepsilon>0$ and its value at $s=0$ is $\operatorname{Tr}\left(v P_{\varphi}\{0\}\right)-\operatorname{Tr}\left(v P_{\varphi+u}\{0\}\right)$.

Proof of Proposition 1.5 (A) Since $\varphi$ satisfies $O p(1)-O p(3)$ and $u$ is bounded, $\varphi+u$ satisfies $O p(1)$ and $O p(2)$ as well. One verifies in a straightforward way that the graph of $\varphi+u$ is closed (and thus $O p(3)$ is valid) and the null space $\operatorname{Ker}(\varphi+u)$ has finite (von Neumann) dimension. It remains to check that $O p(4)$ and $O p(5)$ hold for $\varphi+u$. To see it, notice that, again by the variational characterization of the spectral distribution function, $F_{\varphi+u}(\lambda) \leq F_{\varphi}(\lambda+\|u\|)<$ $\infty \forall \lambda$. Thus

$$
F_{\varphi+u}(\lambda)=0\left(\lambda^{\alpha}\right) \text { for } \lambda \nearrow \infty
$$

where $\alpha$ is given by $O p(5)$, satisfied by $\varphi$, and

$$
F_{\varphi+u}(\lambda)=0(1) \text { for } \lambda \searrow 0
$$

as $\varphi$ satisfies $O p(4)$ and $O p(5)$.

(B) First we prove (B) in the case where $u$ is of the form $u=f(\varphi)$ with $f \in C^{\infty}([0, \infty) ; \mathbb{R})$ being of compact support and such that $\varphi+f(\varphi)$ is 1 1. We claim that $h(t):=\theta_{\varphi+u}(t)-\theta_{\varphi}(t)$ is real analytic near $t=0$ and satisfies $h(0)=\operatorname{dim}(\operatorname{Ker} \varphi)$. From Lemma 1.1 one then concludes that the difference $\zeta_{\varphi}^{\mathrm{I}}(s)-\zeta_{\varphi+f(\varphi)}^{\mathrm{I}}(s)=\frac{1}{\Gamma(s)} \int_{0}^{1} t^{s-1} h(t) d t$ is well defined and holomorphic in $s$ for Res $>\alpha$, has an analytic continuation to Res $>-1$ and takes value $\operatorname{dim}(\operatorname{Ker} \varphi)$ at $s=0$. To prove that $h(t)$ is real analytic in $t$ near $t=0$ and satisfies $h(0)=\operatorname{dim}(\operatorname{Ker} \varphi)$ we argue as follows: Since $\varphi$ and $f(\varphi)$ commute, $e^{-t(\varphi+f(\varphi))}-e^{-t \varphi}=e^{-t \varphi}\left(e^{-t f(\varphi)}-1\right)$. By assumption, $f$ has compact support, i.e. suppf $\subseteq[0, K]$ for some $K>0$. Therefore

$$
f(\varphi)=\int_{0}^{\infty} f(\lambda) d P_{\varphi}(\lambda)=\int_{0}^{K} f(\lambda) d P_{\varphi_{K}}(\lambda)=f\left(\varphi_{K}\right)
$$

where $\varphi_{K}:=\int_{0}^{K} \lambda d P_{\varphi}(\lambda)$ is $\varphi$ restricted to $L:=\overline{\operatorname{range} P([0, K])}$.

Using that $F_{\varphi_{K}}(\lambda)$ is constant for $\lambda>K$, we conclude, integrating by parts,

$$
\begin{aligned}
h_{1}(t):= & \operatorname{Tr} e^{-t \varphi_{K}}\left(e^{-t f\left(\varphi_{K}\right)}-1\right)=\int_{0}^{K} e^{-t \lambda}\left(e^{-t f(\lambda)}-1\right) d F_{\varphi_{K}}(\lambda)= \\
= & \left.e^{-t \lambda}\left(e^{-t f(\lambda)}-1\right) F(\lambda)\right|_{0} ^{K} \\
& -t \int_{0}^{K} e^{-t \lambda}\left(e^{-t f(\lambda)}-1+e^{-t f(\lambda)} f^{\prime}(\lambda)\right) F_{\varphi_{K}}(\lambda) d \lambda
\end{aligned}
$$

which is real analytic in $t$ and satisfies $h_{1}(0)=0$. Further, one obtains

$$
\begin{aligned}
h_{2}(t) & :=\operatorname{Tr}\left(e^{-t\left(\varphi_{K}+f\left(\varphi_{K}\right)\right)} P_{\varphi_{K}+f\left(\varphi_{K}\right)}(\{0\})-e^{-t \varphi_{K}} P_{\varphi_{K}}(\{0\})\right) \\
& =e^{-t f(0)} \operatorname{dim}(\operatorname{Ker}(\varphi+f(\varphi)))-\operatorname{dim}(\operatorname{Ker} \varphi)=-\operatorname{dim}(\operatorname{Ker} \varphi)
\end{aligned}
$$


which is real analytic in $t$ as well and satisfies $h_{2}(0)=-\operatorname{dim} \operatorname{Ker}(\varphi)$. We conclude that $h(t)=h_{1}(t)-h_{2}(t)$ is real analytic in $t$ and $h(0)=\operatorname{dim}(\operatorname{Ker} \varphi)$.

To prove (B) in the general case it suffices, in view of the first step and Lemma 1.4, to consider operators $\varphi$ and $u$ so that, in addition, $\varphi$ and $\varphi+u$ are 1-1 and satisfy $O p(8)$. These additional properties allow to represent $\zeta_{\varphi}^{\mathrm{I}}(s)$ and $\zeta_{\varphi+u}^{\mathrm{I}}(s)$ by a contour integral as follows: Choose $\varepsilon>0$ so that $F_{\varphi}(\lambda)=F_{\varphi+u}(\lambda)=0$ for $0 \leq \lambda<\varepsilon$ and consider the contour $\Gamma_{\varepsilon / 2}=\Gamma_{-} \cup \Gamma_{0} \cup \Gamma_{+}$defined by

$$
\begin{gathered}
\Gamma_{-}:=\left\{z=r e^{-i \pi} \mid \infty>r \geq 0\right\} ; \Gamma_{0}:=\left\{z=\frac{\varepsilon}{2} e^{i \mu} \mid-\pi \leq \mu \leq \pi\right\} ; \\
\Gamma_{+}=\left\{z=r e^{i \pi} \mid \varepsilon / 2 \leq r<\infty\right\} .
\end{gathered}
$$

For $\lambda \in \Gamma_{\varepsilon / 2}, \lambda-\varphi$ and $\lambda-\varphi-u$ are both invertible and one computes

$$
\begin{aligned}
R(\lambda) & :=(\lambda-\varphi-u)^{-1}-(\lambda-\varphi)^{-1}= \\
& =(\lambda-\varphi)^{-1} u(\lambda-\varphi-u)^{-1} .
\end{aligned}
$$

As $u$ is a bounded operator of trace class we conclude by Proposition 1.3 that, for $\lambda \in \Gamma_{\varepsilon / 2}, R(\lambda)$ is a bounded operator of trace class as well. By a standard argument one shows that for $\lambda \in \Gamma_{\varepsilon / 2}$,

$$
\left\|(\lambda-\varphi)^{-1}\right\| \leq \frac{1}{|\lambda-\varepsilon|} \quad\left(\leq \frac{1}{\varepsilon / 2}\right)
$$

$$
\left\|(\lambda-\varphi-u)^{-1}\right\| \leq \frac{1}{|\lambda-\varepsilon|} \quad\left(\leq \frac{1}{\varepsilon / 2}\right) .
$$

These estimates allow to represent the difference $A(s):=\zeta_{\varphi}^{\mathrm{I}}(s)-\zeta_{\varphi+u}^{\mathrm{I}}(s)$ by the following contour integral

$$
A(s)=\frac{1}{2 \pi i} \int_{\Gamma_{\varepsilon / 2}} \lambda^{-s} \operatorname{Tr} R(\lambda) d \lambda=A_{0}(s)+A_{+}(s)+A_{-}(s)
$$

where

$\mathbf{( 1 . 1 4}_{ \pm} \quad A_{ \pm}(s)=\frac{1}{2 \pi i} \int_{\Gamma_{ \pm}} \lambda^{-s} \operatorname{Tr} R(\lambda) d \lambda$

(1.14) $)_{0} \quad A_{0}(s)=\frac{1}{2 \pi i} \int_{\Gamma_{0}} \lambda^{-s} \operatorname{Tr} R(\lambda) d \lambda$.

Notice that $A_{0}(s)$ is holomorphic function for $s \in \mathbb{C}$ and $A_{ \pm}(s)$ are holomorphic functions in a neighborhood of $s=0$ due to the fact that $\int_{\varepsilon / 2}^{\infty} \frac{x^{-s}}{(x+\varepsilon)^{2}} d x$ is absolutely convergent for $|s|$ sufficiently small. Moreover, $A_{0}(0)=0$ and $A_{+}(0)+$ $A(0)=0$. 


\section{Examples 1.6}

1. $\varphi: \mathcal{W}_{1} \rightarrow \mathcal{W}_{2}$ bounded and $\operatorname{dim} \mathcal{W}_{1}<\infty$ : then $\varphi$ satisfies $O p(1)-O p(7)$ and is of trace class. In fact, $O p(6)$ follows from (Asy) which holds with $m=0$ and $\rho=1$. Since $\operatorname{Ker} \varphi \subseteq \mathcal{W}_{1}$ is of finite dimension, $\varphi$ is of $\mathrm{sF}$ type.

2. $\varphi: \mathcal{W}_{1} \rightarrow \mathcal{W}_{2}$ is bounded and $\operatorname{dim} \mathcal{W}_{2}<\infty$ : then the adjoint $\varphi^{*}$ of $\varphi$ is as in Example (1). Therefore $\varphi$ satisfies $O p(1)-O p(6)$ and (Asy) with $m=0, \rho=1$. Moreover $\varphi$ is of trace class. However the dimension of the nullspace $\operatorname{Ker} \varphi$ might not be finite. Therefore, it is useful to reduce $\varphi$ to $\hat{\varphi}: \mathcal{W}_{1} / \operatorname{Ker} \varphi \rightarrow \mathcal{W}_{2}$. Then $\hat{\varphi}$ is injective and bounded and $\operatorname{dim}\left(\mathcal{W}_{1} / \operatorname{Ker} \varphi\right)<\infty$.

3. $\varphi$ of finite rank: then the reduced $\operatorname{map} \hat{\varphi}: \mathcal{W}_{1} / \operatorname{Ker} \varphi \rightarrow \mathcal{W}_{2}$ is bounded and injective and fits either into (1) or (2).

4. Suppose $(\mathrm{M}, \mathrm{g})$ is a closed Riemann manifold and $\mathcal{E} \rightarrow M$ is a smooth bundle of $\mathcal{A}$-Hilbert modules of finite type, equipped with a Hermitian structure $\mu$ and a smooth connection $\nabla$ so that the $\mathcal{A}$-action is parallel. Using $g, \mu$ and $\nabla$ one can define inner products $\langle\cdot, \cdot\rangle_{s}$ of Sobolev type on the space of smooth sections on $\mathcal{E}, C^{\infty}(\mathcal{E})$. (For $s=0$, the connection $\nabla$ is not needed.) The completion of $C^{\infty}(E)$ with respect to $\langle\cdot, \cdot\rangle_{s}$ is a Hilbert space denoted by $H_{s}(\mathcal{E})$. Different choices of $g, \mu, \nabla$ lead to the same topological vector spaces $H_{s}(\mathcal{E})$, with different inner products, but equivalent norms.

A pseudodifferential operator $(\Psi D O)$ of order $d$ induces operators $A: H_{s}(\mathcal{E}) \rightarrow$ $H_{s^{\prime}}(\mathcal{E})$ which are bounded if $s^{\prime} \leq s-d$. The operator $(\Delta+i d)^{-s^{\prime} / 2} A(\Delta+i d)^{s / 2}$ : $L_{2}(\mathcal{E}) \rightarrow L_{2}(\mathcal{E})$ has a kernel of class $C^{k}$ if $s^{\prime}<s-d-k-\operatorname{dim} M$. If $A$ is an elliptic $\Psi D 0$ of order $d>0$ it satisfies $O p(1)-O p(7)$ and (Asy) (with $m:=\operatorname{dim} M$ and $\rho=1 / k)$ cf $[\mathrm{BFKM}]$ section 2 . Hence $A$ is a $\zeta$-regular operator of sF type.

\subsection{Complexes}

In this subsection we introduce the class of $\zeta$-regular complexes of sF type and define their algebraic and reduced cohomology. In the case where a $\zeta$-regular complex of $\mathrm{sF}$ type is algebraically acyclic or, more generally, of determinant class, one can define its torsion. We recall Milnor's lemma which relates the torsions of a short exact sequence of complexes of finite (von Neumann) dimension. Further, within our class of complexes, we discuss the notion of a mapping cone which is a complex induced by a morphism $f$ between two complexes and show that if $f$ is of trace class and induces an isomorphism in algebraic cohomology then the mapping cone is algebraically acyclic. Proposition 1.15 relates, under appropriate conditions, the torsions of the mapping cones of two morphisms and their composition. The class of $\zeta$-regular complexes of $\mathrm{sF}$ type has been chosen so that it includes the mapping cone of a regularized version 
of the integration map between the deRham complex and the combinatorial complex, which will be discussed in section 2 .

A cochain complex $\mathcal{C} \equiv\left(\mathcal{C}_{i}, d_{i}\right)$ of $\mathcal{A}$-Hilbert modules,

$$
\mathcal{C}_{0} \stackrel{d_{0}}{\rightarrow} \mathcal{C}_{1} \stackrel{d_{1}}{\rightarrow} \ldots \rightarrow \mathcal{C}_{N-1} \stackrel{d_{N-1}}{\longrightarrow} \mathcal{C}_{N}
$$

consists of a finite sequence of $\mathcal{A}$-Hilbert modules $\mathcal{C}_{i}(0 \leq i \leq N)$ and operators $d_{i}: \mathcal{C}_{i} \rightarrow \mathcal{C}_{i+1}$, satisfying $O p(1)-O p(3)$, with the property that range $\left(d_{i}\right) \subseteq$ domain $\left(d_{i+1}\right)$ and $d_{i+1} d_{i}=0$.

Notice that the null space $\operatorname{Kerd}_{i}$ is always an $\mathcal{A}$-Hilbert submodule.

Definition 1.7 For $0 \leq i \leq N$ :

algebraic cohomology: $H^{i}(\mathcal{C}):=\operatorname{Kerd}_{i} / \operatorname{range}\left(d_{i-1}\right)$

reduced cohomology $: \bar{H}^{i}(\mathcal{C}):=\operatorname{Kerd}_{i} / \overline{\operatorname{range}\left(d_{i-1}\right)}$.

We point out that the reduced cohomology $\bar{H}^{i}(\mathcal{C})$ is an $\mathcal{A}$-Hilbert module whereas the algebraic cohomology $H^{i}(\mathcal{C})$ is, in general, not an $\mathcal{A}$-Hilbert module, as range $\left(d_{i-1}\right)$ needs not to be closed. $H^{i}(\mathcal{C})$ is always an $\mathcal{A}$ - module.

Given a complex $\left(\mathcal{C}_{i}, d_{i}\right)$ we can define the adjoint operators $d_{i}^{*}: \mathcal{C}_{i+1} \rightarrow \mathcal{C}_{i}$ and, in turn, the Laplacians $\Delta_{i}$,

$$
\Delta_{i}=d_{i}^{*} d_{i}+d_{i-1} d_{i-1}^{*}
$$

as well as the Hodge decomposition $\mathcal{C}_{i}=\mathcal{H}_{i} \oplus \mathcal{C}_{i}^{+} \oplus \mathcal{C}_{i}^{-}$where

$$
\mathcal{H}_{i}:=\operatorname{Ker}\left(d_{i}\right) \cap \operatorname{Ker}\left(d_{i-1}^{*}\right)
$$

$$
\mathcal{C}_{i}^{+}:=\overline{\operatorname{range}\left(d_{i-1}\right)} ; \mathcal{C}_{i}^{-}:=\overline{\operatorname{range}\left(d_{i}^{*}\right)} .
$$

With respect to this decomposition, the operators $d_{i}, d_{i}^{*}$ and $\Delta_{i}$ take the form

$$
\begin{aligned}
d_{i} & =\left(\begin{array}{ccc}
0 & 0 & 0 \\
0 & 0 & \underline{d}_{i} \\
0 & 0 & 0
\end{array}\right) ; d_{i}^{*}=\left(\begin{array}{ccc}
0 & 0 & 0 \\
0 & 0 & 0 \\
0 & \underline{d}_{i}^{*} & 0
\end{array}\right) ; \\
\Delta_{i} & =\left(\begin{array}{ccc}
0 & 0 & 0 \\
0 & \underline{d}_{i-1} \underline{d}_{i-1}^{*} & 0 \\
0 & 0 & \underline{d}_{i}^{*} \underline{d}_{i}
\end{array}\right) .
\end{aligned}
$$


We write $\underline{\Delta}_{i}^{+}:=\underline{d}_{i-1} \underline{d}_{i-1}^{*}$ and $\underline{\Delta}_{i}^{-}:=\underline{d}_{i}^{*} \underline{d}_{i}$ Note that $\underline{d}_{i}: \mathcal{C}_{i}^{-} \rightarrow \mathcal{C}_{i+1}^{+}$and $\underline{d}_{i}^{*}: \mathcal{C}_{i+1}^{+} \rightarrow \mathcal{C}_{i}^{-}$are injective operators with dense image, (hence so are $\underline{\Delta}_{i}^{+}$and $\Delta_{i}^{+}$,)and $\mathcal{H}_{i}$ is isometric to the reduced cohomology $\bar{H}^{i}(\mathcal{C})$.

\section{Definition 1.8}

(i) The cochain complex $\mathcal{C}=\left(\mathcal{C}_{i}, d_{i}\right)$ is said to be of sF type if

(CX1) the operators $\underline{d}_{i}$ satisfy $O p(1)-O p(6)$ (and hence, in view of the injectivity of $\underline{d}_{i}$, are of sF type);

(CX2) $\quad \operatorname{dim} \mathcal{H}_{i}<\infty$.

(ii) A complex $\left(\mathcal{C}_{i}, d_{i}\right)$ of sF type is called $\underline{\zeta \text {-regular }}$ if, in addition, the operators $\underline{d}_{i}$ satisfy $O p(7)$.

(iii) A complex $\left(\mathcal{C}_{i}, d_{i}\right)$ is said to be of finite type (of finite dimension), if the $\mathcal{A}$ Hilbert modules $\mathcal{C}_{i}$ are of finite type (of finite dimension) and the operators $\underline{d}_{i}$ are bounded.

Remark 1 The conditions (CX1) and (CX2) are equivalent to

$\left(C X_{12}\right) \quad$ the Laplacians $\Delta_{i}$ are of sF type

and the three properties (CX1)-(CX3) are equivalent to

$\left(C X_{123}\right) \quad$ the Laplacians $\Delta_{i}$ are $\zeta$-regular operators of sF type.

Remark 2 Assume that $\mathcal{H}_{i_{0}}=0$ for some $i_{0}$. Using the closed graph theorem one verifies that the following statements are equivalent:

(a) $H^{i_{0}}(\mathcal{C})=0$; (b) $\underline{d}_{i_{0}}$ has a bounded inverse.

Given a complex $\left(\mathcal{C}_{i}, d_{i}\right)$ of $\mathrm{sF}$ type, one can define the spectral distribution functions $F_{i}(\lambda)$ and $N_{i}(\lambda)$,

$$
F_{i}(\lambda):=F_{\underline{d}_{i}}(\lambda)\left(=F_{\left|\underline{d}_{i}\right|}(\lambda)\right) ; N_{i}(\lambda):=F_{\Delta_{i}}(\lambda)
$$

Then

$$
N_{i}(\lambda)=F_{i}(\lambda)+F_{i-1}(\lambda) .
$$


A complex $\left(\mathcal{C}_{i}, d_{i}\right)$ of $\mathrm{sF}$ type is said to be algebraically acyclic if $H^{i}(\mathcal{C})=0$ for $0 \leq i \leq N$.

Notice that for an acyclic complex of $\mathrm{sF}$ type, the spectrum of $\Delta_{i}$ does not contain 0 . In fact, in view of Remark 2 after Definition 1.9, there exists $\varepsilon>0$ so that $N_{i}(\lambda)=0$ for $0 \leq \lambda<\varepsilon$ and the same is true for the spectral distribution function of $\underline{\Delta}_{i}^{+}=\underline{d}_{i-1} \underline{d}_{i-1}^{*}$ and $\underline{\Delta}_{i}^{-}=\underline{d}_{i}^{*} \underline{d}_{i}$. This allows to define the torsion $T(\mathcal{C})$ of an algebraically acyclic complex of sF type,

$$
\begin{aligned}
\log T(\mathcal{C}):= & \frac{1}{2} \sum_{q}(-1)^{q+1} q \log \operatorname{det} \Delta_{q} \\
& \left(=\frac{1}{2} \sum_{q}(-1)^{q} \log \operatorname{det} \underline{\Delta}_{q}^{-}=\frac{1}{2} \sum_{q}(-1)^{q+1} \log \operatorname{det} \underline{\Delta}_{q}^{+}\right) .
\end{aligned}
$$

More generally, the torsion $T(\mathcal{C})$ can be defined if $(\mathcal{C}, d)$ is of determinant class, i.e.

$$
\int_{0^{+}}^{1} \log \lambda d N_{i}(\lambda)>-\infty \quad(0 \leq i \leq N)
$$

Definition 1.9 (i) A morphism $f: \mathcal{C}^{1} \rightarrow \mathcal{C}^{2}$ between the complexes $\left(\mathcal{C}_{i}^{1}, d_{1, i}\right)$ and $\left(\mathcal{C}_{i}^{2}, d_{2, i}\right)$ consists of a collection of bounded operators $f_{i}: \mathcal{C}_{i}^{1} \rightarrow \mathcal{C}_{i}^{2}$ so that

- $f_{i}\left(\operatorname{domain}\left(d_{1, i}\right)\right) \subseteq \operatorname{domain}\left(d_{2, i}\right) ; d_{2, i} f_{i}=f_{i+1} d_{1, i}$ (on domain $\left.\left(d_{1, i}\right)\right)$.

(ii) $f$ is said to be of trace class if, in addition,

- the operators $f_{i}, d_{2, i}^{*} f_{i+1}$ and $f_{i} d_{1, i}^{*}$ are bounded operators of trace class.

A morphism $f: \mathcal{C}^{1} \rightarrow \mathcal{C}^{2}$ induces $\mathcal{A}$-linear maps in algebraic cohomology, $H(f) ;: H^{i}\left(\mathcal{C}^{1}\right) \rightarrow H^{i}\left(\mathcal{C}^{2}\right)$ and bounded $\mathcal{A}$-linear maps on the reduced cohomology, $\bar{H}(f)_{i}: \bar{H}^{i}\left(\mathcal{C}^{1}\right) \rightarrow \bar{H}^{i}\left(\mathcal{C}^{2}\right)$. Further, with respect to the Hodge decomposition, $f_{i}$ takes the form,

$$
f_{i}=\left(\begin{array}{lll}
f_{i, 11} & 0 & f_{i, 13} \\
f_{i, 21} & f_{i, 22} & f_{i, 23} \\
0 & 0 & f_{i, 33}
\end{array}\right)
$$

where $f_{i, 11}=\bar{H}(f)_{i}$.

For the convenience of the reader, we recall Milnor's lemma which was extended for complexes of $\mathcal{A}$-Hilbert modules in [BFK2]. Assume that

$$
0 \rightarrow \mathcal{C}^{1} \stackrel{f}{\rightarrow} \mathcal{C}^{2} \stackrel{g}{\rightarrow} \mathcal{C}^{3} \rightarrow 0
$$


is a short exact sequence of complexes of finite dimension. It induces a long weakly exact sequence $\mathcal{H}$ in reduced cohomology

$$
\ldots \rightarrow \bar{H}^{i}\left(\mathcal{C}^{1}\right) \stackrel{\bar{H}\left(f_{i}\right)}{\longrightarrow} \bar{H}^{i}\left(\mathcal{C}^{2}\right) \stackrel{\bar{H}\left(g_{j}\right)}{\longrightarrow} \bar{H}^{i}\left(\mathcal{C}^{3}\right) \stackrel{\bar{H}\left(\delta_{j}\right)}{\longrightarrow} \bar{H}^{i+1}\left(\mathcal{C}^{1}\right) \rightarrow \ldots
$$

Proposition 1.10 (cf [BFK2, Theorem 1.5]) If three out of the four cochain complexes $\mathcal{C}^{1}, \mathcal{C}^{2}, \mathcal{C}^{3}, \mathcal{H}$ are of determinant class, then so is the fourth and one has the following equality

$$
\begin{aligned}
\log T\left(\mathcal{C}^{2}\right) & =\log T\left(\mathcal{C}^{1}\right)+\log T\left(\mathcal{C}^{3}\right)+\log T(\mathcal{H})- \\
& -\sum_{i}(-1)^{i} \log T\left(0 \rightarrow \mathcal{C}_{i}^{1} \rightarrow \mathcal{C}_{i}^{2} \rightarrow \mathcal{C}_{i}^{3} \rightarrow 0\right) .
\end{aligned}
$$

Remark In [BFK2], Lemma B2.8 was only stated for complexes of finite type. By the same proof, the result remains true for complexes of finite dimension.

Given a $\zeta$-regular complex of sF type, $0 \rightarrow \mathcal{C}_{0} \stackrel{d_{Q}}{\rightarrow} \ldots \stackrel{d_{2 N}}{\rightarrow} \mathcal{C}_{2 N+1} \rightarrow 0$, we can consider its dual. If $\mathcal{C}_{j}^{\sharp}:=\mathcal{C}_{2 N+1-j}$, and $d_{j}^{\sharp}:=d_{2 N-j}^{*}$ (adjoint of $d_{2 N-j}$ ), then

$$
0 \rightarrow \mathcal{C}_{0}^{\sharp} \stackrel{d_{0}^{\sharp}}{\rightarrow} \ldots \stackrel{d_{2 N}^{\sharp}}{\rightarrow} \mathcal{C}_{2 N+1}^{\sharp} \rightarrow 0
$$

is a $\zeta$-regular complex of sF type. Notice that $\left(\underline{d}_{j}^{\sharp}\right)^{*} \underline{d}_{i}^{\sharp}=\underline{d}_{2 N-j} \underline{d}_{2 N-j}^{*}$. Thus in case $\mathcal{C}$ is of determinant class, so is $\mathcal{C}^{\sharp}$ and one obtains

$$
T\left(\mathcal{C}^{\sharp}\right)=T(\mathcal{C}) .
$$

We end this subsection with a discussion of the mapping cone $\mathcal{C}(f)=\left(\mathcal{C}(f)_{i}\right.$, $\left.d(f)_{i}\right)$ associated to a morphism $f: \mathcal{C}^{1} \rightarrow \mathcal{C}^{2}$ between $\zeta$-regular complexes $\left(\mathcal{C}^{1}, d_{1}\right)$ and $\left(\mathcal{C}^{2}, d_{2}\right)$ of $\mathrm{sF}$ type. This is a complex given by

$(\mathrm{MC} 1) \mathcal{C}(f)_{i}:=\mathcal{C}_{i-1}^{2} \oplus \mathcal{C}_{i}^{1}$

$$
d(f)_{i}=\left(\begin{array}{cc}
-d_{2, i-1} & f_{i} \\
0 & d_{1, i}
\end{array}\right)
$$

The Laplacians $\Delta(f)_{i}$ of $\mathcal{C}(f)$ can be computed,

$$
\Delta(f)_{i}=\left(\begin{array}{cc}
\Delta_{2, i-1}+f_{i-1} f_{i-1}^{*} & -d_{2, i-1}^{*} \cdot f_{i}+f_{i-1} \cdot d_{1, i-1}^{*} \\
-f_{i}^{*} \cdot d_{2, i-1}+d_{1, i-1} \cdot f_{i-1}^{*} & \Delta_{1, i}+f_{i}^{*} f_{i}
\end{array}\right)
$$

The following observation, will be used in order to introduce the relative $L_{2}$ torsion without making the assumption that the manifold is of determinant class: 
Lemma 1.11 (i) Let $f: \mathcal{C}^{1} \rightarrow \mathcal{C}^{2}$ be a morphism of trace class between complexes $\mathcal{C}^{1}$ and $\mathcal{C}^{2}$ of $s F$ type. Then the mapping cone $\mathcal{C}(f)$ is of $s F$ type.

(ii) If, in addition, $\mathcal{C}^{1}$ and $\mathcal{C}^{2}$ are $\zeta$-regular, then $\mathcal{C}(f)$ is $\zeta$-regular.

(iii) If $\varphi: \mathcal{C}^{1} \rightarrow \mathcal{C}^{2}$ is a morphism of trace class between $\zeta$-regular complexes of sF type which induces an isomorphism in algebraic cohomology, then the mapping cone $\mathcal{C}(f)$ is algebraically acyclic and thus has a well defined torsion $T(\mathcal{C}(f))(c f(1.21))$.

Proof (i) follows from Proposition 1.5 (A) by choosing $\varphi:=\left(\begin{array}{cc}\Delta_{2, i-1} & 0 \\ 0 & \Delta_{1, i}\end{array}\right)$ and

$$
u_{i}:=\Delta(f)_{i}-\varphi_{i}=\left(\begin{array}{cc}
f_{i-1} f_{i-1}^{*} & -d_{2, i-1}^{*} f_{i}+f_{i-1} d_{1, i-1}^{*} \\
-f_{i}^{*} d_{2, i-1}+d_{1, i-1} f_{i-1}^{*} & f_{i}^{*} f_{i}
\end{array}\right)
$$

By Remark 1 after Definition 1.8, the $\varphi_{i}$ are operators of $\mathrm{sF}$ type and, by assumption, the $u_{i}$ are bounded operators of trace class. By Proposition 1.5, $\Delta(f)_{i}$ is then an operator of $\mathrm{sF}$ type. Apply the same Remark 1 once more to conclude that (CX1) holds. Property (CX2) of Definition 1.8 is easily verified.

(ii) follows from Proposition 1.5 (B) and Remark 1 after Definition 1.8.

(iii) In view of (1.21) and the statements (i) and (ii) it remains to verify that $\mathcal{C}(f)$ is algebraically acyclic, i.e. that range $\left(d(f)_{i-1}\right)=\operatorname{Ker}\left(d(f)_{i}\right)$. Let $\left(v_{i-1}, u_{i}\right) \in$ $\operatorname{Ker}\left(d(f)_{i}\right) \subseteq \mathcal{C}_{i-1}^{2} \oplus \mathcal{C}_{i}^{1}$. Then

$$
-d_{2, i-1} v_{i-1}+f_{i} u_{i}=0 ; \quad d_{1, i} u_{i}=0 .
$$

In particular, $f_{i} u_{i} \in$ range $\left(d_{2, i-1}\right)$ and, as $f$ induces an isomorphism in algebraic cohomology, $u_{i} \in \operatorname{range}\left(d_{1, i-1}\right)$, i.e. there exists $u_{i-1} \in \mathcal{C}_{i-1}^{1}$ with $u_{i}=d_{1, i-1}$ $u_{i-1}$. As $f_{i} d_{1, i-1} u_{i-1}=d_{2, i-1} f_{i-1} u_{i-1}$, we conclude that $-v_{i-1}+f_{i-1} u_{i-1} \in$ $\operatorname{Kerd}_{2, i-1}$. Using once again the assumption that $f$ induces an isomorphism in algebraic cohomology, one sees that there exists $v_{i-2} \in \mathcal{C}_{i-2}^{2}$ and $\tilde{u}_{i-1} \in$ $\operatorname{Kerd}_{1, i-1}$ with $-v_{i-1}+f_{i-1} u_{i-1}=f_{i-1} \tilde{u}_{i-1}+d_{2, i-2} v_{i-2}$.

Thus

$$
v_{i-1}=-d_{2, i-2} v_{i-2}+f_{i-1}\left(u_{i-1}-\tilde{u}_{i-1}\right)
$$

and, as $\tilde{u}_{i-1} \in \operatorname{Kerd}_{1, i-1}$

$$
u_{i}=d_{1, i-1} u_{i-1}=d_{1, i-1}\left(u_{i-1}-\tilde{u}_{i-1}\right)
$$


i.e. we have shown that $\left(v_{i-1}, v_{i}\right) \in \operatorname{ranged}(f)_{i-1}$, and therefore that $\operatorname{Kerd}(f)_{i}$ $=\operatorname{ranged}(f)_{i-1}$.

If $f:\left(\mathcal{C}, d_{1}\right) \rightarrow\left(\mathcal{C}, d_{2}\right)$ is an isomorphism of complexes of finite dimension we can use Lemma 1.11 together with Milnor's lemma to compute the torsion of the mapping cone $\mathcal{C}(f)$. Recall that the suspension $\Sigma \mathcal{C}=\left(\Sigma \mathcal{C}_{i, \Sigma} d_{i}\right)$ of a complex $\mathcal{C}=\left(\mathcal{C}_{i}, d_{i}\right)$ is the complex given by

$$
\begin{aligned}
& \Sigma \mathcal{C}_{i} \equiv(\Sigma \mathcal{C})_{i}:=\mathcal{C}_{i-1}(i \geq 1) ; \Sigma \mathcal{C}_{0}:=0 \\
& \Sigma d_{i}:=-d_{i-1}(i \geq 1) ; d_{0}=0
\end{aligned}
$$

Proposition 1.12 Assume that $\mathcal{C}^{i} \equiv\left(\mathcal{C}, d_{j}\right)(j=1,2)$ are $\zeta$-regular complexes of $s F$ type and of finite dimension. If $f: \mathcal{C}^{1} \rightarrow \mathcal{C}^{2}$, is an isomorphism, then the mapping cone $\mathcal{C}(f)$ is algebraically acyclic and

$$
\log T(\mathcal{C}(f))=\sum(-1)^{j} \log \operatorname{vol}\left(f_{j}\right)
$$

Proof By Lemma 1.11, $\mathcal{C}(f)$ is an algebraically $\zeta$-regular complex of sF type.

First we treat the case where, in addition, $\mathcal{C}^{1}$ (and then $\mathcal{C}^{2}$ as well) is algebraically acyclic. Consider the following short exact sequence of cochain complexes

$$
0 \rightarrow \Sigma\left(\mathcal{C}, d_{2}\right) \stackrel{J}{\rightarrow} \mathcal{C}(f) \stackrel{P}{\rightarrow}\left(\mathcal{C}, d_{1}\right) \rightarrow 0
$$

where $J$ (respectively $\mathrm{P}$ ) is the canonical inclusion (canonical projection).

By Proposition 1.10, the corresponding long weakly exact sequence $\mathcal{H}$ in reduced cohomology is of determinant class and

$$
\log T(\mathcal{C}(f))=\log T\left(\Sigma \mathcal{C}^{2}\right)+\log T\left(\mathcal{C}^{1}\right)+\log T(\mathcal{H}) .
$$

Notice that $\mathcal{H}$ is given by

$$
\ldots \rightarrow \bar{H}^{i}(\Sigma \mathcal{C}) \rightarrow \bar{H}^{i}(\mathcal{C}(f)) \rightarrow \bar{H}^{i}(\mathcal{C}) \stackrel{\bar{H}\left(\delta_{i}\right)}{\longrightarrow} \bar{H}^{i+1}(\Sigma \mathcal{C}) \rightarrow \ldots
$$

where the connecting homomorphism $\bar{H}\left(\delta_{i}\right)$ is given by the restriction $\bar{H}\left(f_{i}\right)$ of $f_{i}$ to $\bar{H}^{i+1}(\Sigma \mathcal{C})=\bar{H}^{i}(\mathcal{C})$ into $\bar{H}^{i}(\mathcal{C})$. Therefore

$$
\log T(\mathcal{H})=\sum(-1)^{i} \log \operatorname{vol}\left(\bar{H}\left(f_{i}\right)\right) .
$$

By Lemma 1.13 below, $\log T\left(\Sigma \mathcal{C}^{2}\right)=-\log T\left(\mathcal{C}^{2}\right)$. As $f_{i+1} d_{1, i}=d_{2, i} f_{i}$ we conclude that $\underline{d}_{1, i}=\left(f_{i+1}^{+}\right)^{-1} \underline{d}_{2, i} f_{i}^{-}$where $f_{i}^{ \pm}: \mathcal{C}_{i}^{1, \pm} \rightarrow \mathcal{C}_{i}^{2, \pm}$ are the restrictions of $f_{i}$ to $\mathcal{C}_{i}^{1, \pm}$ and are isomorphisms as well. 
Thus

$$
\begin{aligned}
\log T\left(\mathcal{C}^{1}\right) & =\frac{1}{2} \sum(-1)^{i} \log \operatorname{det}\left(\underline{d}_{1, i}^{*} \underline{d}_{1, i}\right) \\
& =\frac{1}{2} \sum(-1)^{i} \log \operatorname{det}\left(\left(f_{i}^{-}\right)^{*} \underline{d}_{2, i}^{*}\left(\left(f_{i+i}^{+}\right)^{-1}\right)^{*}\left(f_{i+1}^{+}\right)^{-1} \underline{d}_{2, i} f_{i}^{-}\right) \\
& =\frac{1}{2} \sum(-1)^{i} \log \operatorname{det}\left(f_{i+1}^{+} f_{i+1}^{+*}\right)^{-1} \\
& +\frac{1}{2} \sum(-1)^{i} \log \operatorname{det}\left(\underline{d}_{2, i}^{*} \underline{d}_{2, i}\right) \\
& +\frac{1}{2} \sum(-1)^{i} \log \operatorname{det}\left(f_{i}^{-} f_{i}^{-*}\right) \\
& =\log T\left(\mathcal{C}^{2}\right)+\sum_{i}(-1)^{i}\left(\log \operatorname{vol}\left(f_{i}^{+}\right)+\log \operatorname{vol}\left(f_{i}^{-}\right)\right) .
\end{aligned}
$$

Combining the equality above yields

$$
\begin{aligned}
\log T(\mathcal{C}(f)) & =\sum(-1)^{i}\left(\log \operatorname{vol}\left(f_{i}^{+}\right)+\log \operatorname{vol}\left(f_{i}^{-}\right)\right) \\
& +\sum(-1)^{i} \log \operatorname{vol}\left(\bar{H}\left(f_{i}\right)\right) \\
& =\sum(-1)^{i} \log \operatorname{vol}\left(f_{i}\right) .
\end{aligned}
$$

To prove the result in general we consider a deformation $\left(\mathcal{C}, d_{1}(\varepsilon)\right)$ of the complex $\left(\mathcal{C}, d_{1}\right)$, depending smoothly on the parameter $\varepsilon$, so that, for $\varepsilon \neq 0,\left(\mathcal{C}, d_{1}(\varepsilon)\right)$ is algebraically acyclic. The operator $d_{1}(\varepsilon)$ is constructed as follows: with respect to the Hodge decomposition $\mathcal{C}_{i}=\mathcal{H}_{i} \oplus \mathcal{C}_{i}^{+} \oplus \mathcal{C}_{i}^{-}, d_{1, i}$ takes the form $d_{1, i}=\left(\begin{array}{ccc}0 & 0 & 0 \\ 0 & 0 & \underline{d}_{1, i} \\ 0 & 0 & 0\end{array}\right)$ where $\underline{d}_{1, i}: \mathcal{C}_{1, i}^{-} \rightarrow \mathcal{C}_{1, i+1}^{+}$. Consider the polar decomposition of $\underline{d}_{1, i}, \underline{d}_{1, i}=\xi_{i}, \eta_{i}$ where $\xi_{i}:=\underline{d}_{1, i}\left(\underline{d}_{1, i}^{*} \underline{d}_{1, i}\right)^{-1 / 2}: \mathcal{C}_{i}^{-} \rightarrow \mathcal{C}_{i+1}^{+}$is an isometry and $\eta_{i}$ is given by $\eta_{i}:=\left(\underline{d}_{1, i}^{*} \underline{d}_{1, i}\right)^{1 / 2}$. The operator $\eta_{i}$ admits a spectral decomposition; $\eta_{i}=\int_{0^{+}}^{\infty} \lambda d P_{i}(\lambda)$. Define $\eta_{i}(\varepsilon):=\int_{0^{+}}^{\infty}(\lambda+\varepsilon) d P_{i}(\lambda)$ and let $\underline{d}_{1, i}(\varepsilon):=\xi_{i} \eta_{i}(\varepsilon)$. Then $\underline{d}_{1, i}(\varepsilon)$ is an isomorphism and thus of determinant class. Now consider $\mathcal{C}_{\varepsilon}^{1} \equiv\left(\mathcal{C}, \underline{d}_{1},(\varepsilon)\right)$ where the operator $d_{1, i}(\varepsilon)$, with respect to the Hodge decomposition of $\left(\mathcal{C}, d_{1}\right)$, is given by $d_{1, i}(\varepsilon)=\left(\begin{array}{lll}0 & 0 & 0 \\ 0 & 0 & \underline{d}_{1, i}(\varepsilon) \\ 0 & 0 & 0\end{array}\right)$. Define $\left(\mathcal{C}, d_{2}(\varepsilon)\right)$ where $\underline{d}_{2, i}(\varepsilon):=f_{i+1}^{+} \underline{d}_{1, \varepsilon}\left(f_{i}^{-}\right)^{-1}$. Then, for any $\varepsilon, f:\left(\mathcal{C}, d_{1}(\varepsilon)\right) \rightarrow$ $\left(\mathcal{C}, d_{2}(\varepsilon)\right)$ is a morphism and, by the argument above, the torsion $T\left(\mathcal{C}_{\varepsilon}(f)\right)$ of the mapping cone $\mathcal{C}_{\varepsilon}(f)$ induced by $f:\left(\mathcal{C}, d_{1}(\varepsilon)\right) \rightarrow\left(\mathcal{C}, d_{2}(\varepsilon)\right)$ is given by, for $\varepsilon \neq 0$,

$$
\log T\left(\mathcal{C}_{\varepsilon}(f)\right)=\sum(-1)^{i} \log \operatorname{vol}\left(f_{i}\right)
$$

which is independent of $\varepsilon$. As $\mathcal{C}_{\varepsilon}(f)$ is algebraically acyclic for all values of $\varepsilon$ and $\log T\left(\mathcal{C}_{\varepsilon}(f)\right)$ depends continuously on $\varepsilon$, we conclude that

$$
\log T(\mathcal{C}(f))=\sum_{j}(-1)^{j} \log \operatorname{vol}\left(f_{j}\right) .
$$


Lemma 1.13 (i) Let $\mathcal{C}$ be a $\zeta$-regular complex of sF type. Then the mapping cone $\mathcal{C}(i d)$ is an algebraically acyclic $\zeta$-regular complex of sF type and satisfies $\log T(\mathcal{C}(i d))=0$.

(ii) If $\mathcal{C}$ is an algebraically acyclic, $\zeta$-regular complex of sF type, then so is $\Sigma \mathcal{C}$ and satisfies

$$
\log T(\Sigma \mathcal{C})=-\log T(\mathcal{C})
$$

Proof (i) By Lemma 1.11, $\mathcal{C}(i d)$ is an algebraically acyclic $\zeta$-regular complex of $\mathrm{sF}$ type. Use Lemma 2.4 to conclude that

$$
\log T(\mathcal{C}(i d))=\frac{1}{2} \sum(-1)^{q+1} \log \operatorname{det}\left(\Delta_{q}+i d\right)=0 .
$$

(ii) It follows from Definition 1.9 and the assumptions that $\Sigma \mathcal{C}$ is an algebraically acyclic, $\zeta$-regular complex of $\mathrm{sF}$ type. Its torsion is therefore well defined, and by (1.21)

$$
\begin{aligned}
\log T(\Sigma \mathcal{C}) & =\frac{1}{2} \sum(-1)^{q+1} \log \operatorname{det}\left(\underline{\Delta(\Sigma \mathcal{C}}_{q}^{-}\right) \\
& =-\frac{1}{2} \sum(-1)^{q+1} \log \operatorname{det}\left(\underline{\Delta}_{q}^{-}\right)=-\log T(\mathcal{C})
\end{aligned}
$$

The following Lemma 1.14 is due to Carey, Mathai and Mishchenko [CMM]. For the convenience of the reader we include its statement. As the proof in the preliminary preprint $[\mathrm{CMM}]^{2}$ is somewhat incomplete, we refer the reader to Appendix A for a detailed proof. Let

$$
0 \rightarrow \mathcal{C}^{1} \stackrel{I}{\rightarrow} \mathcal{C} \stackrel{P}{\rightarrow} \mathcal{C}^{2} \rightarrow 0
$$

be an exact sequence of cochain complexes of $\mathrm{sF}$ type where the complex $\mathcal{C}=$ $\left(\mathcal{C}_{i}, d_{i}\right)$ is given by $\mathcal{C}_{i}=\mathcal{C}_{i}^{1} \oplus \mathcal{C}_{i}^{2}$ and

$$
d_{i}=\left(\begin{array}{ll}
d_{1, i} & f_{i} \\
0 & d_{2, i}
\end{array}\right)
$$

with $f_{i}: \mathcal{C}_{i}^{2} \rightarrow \mathcal{C}_{i+1}^{1}$ satisfying

$$
f_{i}\left(\operatorname{domain}\left(d_{2, i}\right)\right) \subseteq \operatorname{domain}\left(d_{1, i+1}\right) \text { and }
$$

$$
f_{i+1} d_{2, i}+d_{1, i+1} f_{i}=0 \quad \text { (on domain }\left(d_{2, i}\right) \text { ) }
$$

\footnotetext{
${ }^{2}$ The first author thanks Mishchenko for kindly making the preliminary preprint available to him.
} 
so that $d_{i+1} d_{i}=0$. The morphism I [resp. $\mathrm{P}$ ] in (1.24) denotes the canonical inclusion [resp. canonical projection].

Lemma 1.14 ([CMM]) Assume $\mathcal{C}^{1}$ and $\mathcal{C}^{2}$ are $\zeta$-regular complexes of sF type which are algebraically acyclic and $f_{i}: \mathcal{C}_{i}^{2} \rightarrow \mathcal{C}_{i+1}^{1}$ are bounded maps of trace class so that (1.26) and (1.27) are satisfied and $d_{1, i}^{*} f_{i}$ as well as $f_{i} d_{2, i}^{*}$ are bounded operators of trace class. Then the complex $\mathcal{C}$, given by (1.24) - (1.25), is an algebraically acyclic, $\zeta$-regular complex of sF type and

$$
\log T(\mathcal{C})=\log T\left(\mathcal{C}^{1}\right)+\log T\left(\mathcal{C}^{2}\right) .
$$

Proof: $\mathrm{cf}[\mathrm{CMM}]$ or Appendix A.

Proposition 1.15 Suppose that $\mathcal{C}^{1}, \mathcal{C}^{2}$ and $\mathcal{C}^{3}$ are $\zeta$-regular complexes of $s F$ type and $f_{1}: \mathcal{C}^{1} \rightarrow \mathcal{C}^{2}$ and $f_{2}: \mathcal{C}^{2} \rightarrow \mathcal{C}^{3}$ are morphisms of trace class which induce isomorphism in algebraic cohomology. Then:

(i) $f:=f_{2} \circ f_{1}$ is a morphism of trace class;

(ii) the mapping cones $\mathcal{C}\left(f_{1}\right), \mathcal{C}\left(f_{2}\right)$ and $\mathcal{C}(f)$ are $\zeta$-regular complexes of $s F$ type;

(iii) $\mathcal{C}\left(f_{1}\right), \mathcal{C}\left(f_{2}\right)$ and $\mathcal{C}(f)$ are algebraically acyclic (and thus their torsions are well defined).

(iv) If, in addition, either $\mathcal{C}^{3}$ (case 1) or $\mathcal{C}^{1}$ (case 2) is of finite type, then $\log T(\mathcal{C}(f))=\log T\left(\mathcal{C}\left(f_{1}\right)\right)+\log T\left(\mathcal{C}\left(f_{2}\right)\right)$.

Proof The proof is an application of Lemma 1.13 and Lemma 1.14.

Case $1\left(\mathcal{C}^{3}\right.$ is of finite type): Consider the diagram 


$$
\begin{array}{cc}
\Sigma \mathcal{C}\left(i d_{3}\right) & \\
& \downarrow I_{1} \\
\Sigma \mathcal{C}\left(-f_{2}\right) \stackrel{I_{2}}{\rightarrow} \quad & \mathcal{C}(h) \quad \stackrel{P_{2}}{\rightarrow} \mathcal{C}(f) \rightarrow 0 \\
& \downarrow P_{1} \\
& \\
& \mathcal{C}\left(f_{1}\right) \\
& \downarrow \\
&
\end{array}
$$

where $\mathcal{C}\left(f_{1}\right), \mathcal{C}\left(-f_{2}\right), \mathcal{C}(f)$ and $\mathcal{C}\left(i d_{3}\right)$ are mapping cones, hence $\mathcal{C}\left(f_{1}\right) ;=\mathcal{C}_{i-1}^{2} \oplus$ $\mathcal{C}_{i}^{1}, \mathcal{C}\left(-f_{2}\right)_{i}=\mathcal{C}_{i-1}^{3} \oplus \mathcal{C}_{i}^{2}, \mathcal{C}(f)_{i}=\mathcal{C}_{i-1}^{3} \oplus \mathcal{C}_{i}^{1}$ and $\mathcal{C}\left(i d_{3}\right)_{i}=\mathcal{C}_{i-1}^{3} \oplus \mathcal{C}_{i}^{3}$. The morphism $h: \mathcal{C}\left(f_{1}\right) \rightarrow \mathcal{C}\left(i d_{3}\right)$, is given by the operators $h_{i}: \mathcal{C}_{i-1}^{2} \oplus \mathcal{C}_{i}^{1} \rightarrow$ $\mathcal{C}_{i-1}^{3} \oplus \mathcal{C}_{i}^{3}$,

$$
h_{i}:=\left(\begin{array}{cc}
f_{2, i-1} & 0 \\
0 & f_{i}
\end{array}\right),
$$

One verifies that $h_{i}$ are bounded maps of trace class. Denote by $\mathcal{C}(h)$ the mapping cone of $h$, hence $\mathcal{C}(h)_{i}=\left(\mathcal{C}_{i-2}^{3} \oplus \mathcal{C}_{i-1}^{3}\right) \oplus\left(\mathcal{C}_{i-1}^{2} \oplus \mathcal{C}_{i}^{1}\right)$. Further $I_{1}$, respectively $I_{2}$, are the canonical inclusions,

$$
I_{1}: \mathcal{C}_{i-2}^{3} \oplus \mathcal{C}_{i-1}^{3} \rightarrow\left(\mathcal{C}_{i-2}^{3} \oplus \mathcal{C}_{i-1}^{3}\right) \oplus\left(\mathcal{C}_{i-1}^{2} \oplus \mathcal{C}_{i}^{1}\right)
$$

and

$$
I_{2}: \mathcal{C}_{i-2}^{3} \oplus \mathcal{C}_{i-1}^{2} \rightarrow\left(\mathcal{C}_{i-2}^{3} \oplus \mathcal{C}_{i-1}^{3}\right) \oplus\left(\mathcal{C}_{i-1}^{2} \oplus \mathcal{C}_{i}^{1}\right)
$$

whereas $P_{1}$ and $P_{2}$ denote the canonical projections on the complement of the images of $I_{1}$ resp. $I_{2}$. One verifies (cf (1.29)) that

$$
0 \rightarrow \Sigma \mathcal{C}\left(i d_{3}\right) \stackrel{I_{1}}{\rightarrow} \mathcal{C}(h) \stackrel{P_{1}}{\rightarrow} \mathcal{C}\left(f_{1}\right) \rightarrow 0
$$

and

$$
0 \rightarrow \Sigma \mathcal{C}\left(-f_{2}\right) \stackrel{I_{2}}{\rightarrow} \mathcal{C}(h) \stackrel{P_{3}}{\rightarrow} \mathcal{C}(f) \rightarrow 0
$$

are short exact sequences of cochain complexes. First notice that by Proposition $1.3, f=f_{2} \cdot f_{1}$ is a morphism of trace class which proves (i).

By Lemma $1.11, \mathcal{C}\left(f_{1}\right), \mathcal{C}\left(f_{2}\right)$ and $\mathcal{C}(f)$ are algebraically acyclic, $\zeta$-regular complexes of $\mathrm{sF}$ type and, by Lemma $1.13, \mathcal{C}\left(i d_{3}\right)$ and $\Sigma \mathcal{C}\left(f_{i}\right), \Sigma \mathcal{C}\left(f_{2}\right), \Sigma \mathcal{C}\left(i d_{3}\right)$ are 
algebraically acyclic, $\zeta$-regular complex of $\mathrm{sF}$ type. We would like to apply Lemma 1.14 to (1.31) and (1.32). For this purpose, observe that, given $h_{i}$ : $\mathcal{C}\left(f_{1}\right)_{i}=\mathcal{C}_{i-1}^{2} \oplus \mathcal{C}_{i} \rightarrow \mathcal{C}\left(i d_{3}\right)=\mathcal{C}_{i-1}^{3} \oplus \mathcal{C}_{i}^{3}, d(h)_{i}$ admits the following decomposition

$$
\begin{aligned}
d(h)_{i} & =\left(\begin{array}{c|cc}
d\left(\Sigma \mathcal{C}\left(i d_{3}\right)\right)_{i} & h_{i} \\
\hline 0 & d\left(\mathcal{C}\left(f_{1}\right)_{i}\right.
\end{array}\right), \\
& =\left(\begin{array}{cc|cc}
d_{3, i-2} & -i d_{3, i-1} & f_{2, i-1} & 0 \\
0 & -d_{3, i-1} & 0 & f_{i} \\
\hline 0 & 0 & d_{2, i-1} & f_{1, i} \\
0 & 0 & 0 & d_{1, i}
\end{array}\right)
\end{aligned}
$$

Using this decomposition, all assumptions in Lemma 1.15 which have not yet been proved can be verified in a straight forward way.

Therefore, we can apply Lemma 1.14 to $(1.31)$ to conclude that $\mathcal{C}(h)$ is an algebraically acyclic, $\zeta$-regular complex of $\mathrm{sF}$ type and

$$
\begin{aligned}
\log T(\mathcal{C}(h)) & =\log T\left(\Sigma \mathcal{C}\left(i d_{3}\right)\right)+\log T\left(\mathcal{C}\left(f_{1}\right)\right) \\
& =\log T\left(\mathcal{C}\left(f_{1}\right)\right)
\end{aligned}
$$

where we used that, by Lemma 1.13,

$$
\log T\left(\Sigma \mathcal{C}\left(i d_{3}\right)\right)=-\log T\left(\mathcal{C}\left(i d_{3}\right)\right)=0
$$

In order to apply Lemma 1.14 to (1.32), notice that

$$
\left(\begin{array}{c|c}
d\left(\Sigma \mathcal{C}\left(-f_{2}\right)\right)_{i} & \tilde{h}_{i} \\
\hline 0 & d(\mathcal{C}(f))_{i}
\end{array}\right) \quad=
$$

$$
\left(\begin{array}{cc|cc}
d_{3, i-2} & +f_{2, i-1} & \tilde{h}_{i} & \\
0 & -d_{2, i-1} & & \\
\hline 0 & 0 & -d_{3, i-1} & f_{i} \\
0 & 0 & 0 & d_{1, i}
\end{array}\right)=d(h)_{i}
$$

where, in view of (1.33), $\tilde{h}_{i}: \mathcal{C}(f)_{i}=\mathcal{C}_{i-1}^{3} \oplus \mathcal{C}_{i}^{1} \rightarrow \mathcal{C}_{i-1}^{3} \oplus \mathcal{C}_{i}^{2}=\Sigma \mathcal{C}\left(-f_{2}\right)_{i+1}$ given by $\tilde{h}_{i}=\left(\begin{array}{cc}-i d_{3, i-1} & 0 \\ 0 & f_{1, i}\end{array}\right)$. As $\mathcal{C}^{3}$ is of finite type, $i d_{3, i}: \mathcal{C}_{i}^{3} \rightarrow \mathcal{C}_{i}^{3}$ and therefore $\tilde{h}_{i}$ are bounded maps of trace class. Again one verifies that all other assumption in Lemma 1.14 are satisfied. Thus we can apply Lemma 1.14 to (1.32), to conclude that

$$
\begin{aligned}
\log T(\mathcal{C}(h)) & =\log T\left(\Sigma \mathcal{C}\left(-f_{2}\right)\right)+\log T(\mathcal{C}(f)) \\
& =-\log T\left(\mathcal{C}\left(-f_{2}\right)\right)+\log T(\mathcal{C}(f))
\end{aligned}
$$


where for the last identity we again used Lemma 1.13. Notice that the morphism $\Phi: \mathcal{C}\left(f_{2}\right) \rightarrow \mathcal{C}\left(-f_{2}\right)$, given by $\Phi_{i}=\left(\begin{array}{cc}I d & 0 \\ 0 & -I d\end{array}\right): \mathcal{C}_{i-1}^{3} \oplus \mathcal{C}_{i}^{2} \rightarrow \mathcal{C}_{i-1}^{3} \oplus \mathcal{C}_{i}^{2}$, is an isometry and therefore $\log T\left(\mathcal{C}\left(-f_{2}\right)\right)=\log T\left(\mathcal{C}\left(f_{2}\right)\right)$. Combining this with (1.34) and (1.36) we conclude that statement (iv) in case 1 is proved.

Case $2\left(\mathcal{C}^{1}\right.$ of finite type): In view of (1.22) it suffices to consider the dual complexes, to which we can apply case 1 .

Proposition 1.16 Suppose that $\mathcal{C}^{1}, \mathcal{C}^{2}$ and $\mathcal{C}^{3}$ are $\zeta$-regular complexes of $s F$ type and $f_{1}: \mathcal{C}^{1} \rightarrow \mathcal{C}^{2}, f_{2}: \mathcal{C}^{2} \rightarrow \mathcal{C}^{3}$ are morphisms which induce isomorphisms in algebraic cohomology and denote by $f$ the composition $f:=f_{2} \circ f_{1}$. Then the following statements hold:

(i) If $f_{1}$ is an isometry and $f_{2}$ is of trace class, then the mapping cones $\mathcal{C}\left(f_{2}\right)$ and $\mathcal{C}(f)$ are algebraically acyclic $\zeta$-regular complexes of $s F$ type. Moreover

$$
\log T\left(\mathcal{C}\left(f_{2}\right)\right)=\log T(\mathcal{C}(f))
$$

(ii) If $f_{2}$ is an isometry and $f_{1}$ is of trace class, then $\mathcal{C}\left(f_{1}\right)$ and $\mathcal{C}(f)$ are algebraically acyclic $\zeta$-regular complexes of sF type. Moreover

$$
\log T\left(\mathcal{C}\left(f_{1}\right)\right)=\log T(\mathcal{C}(f))
$$

Proof The assumption imply that in case $(\mathrm{i}), \mathcal{C}\left(f_{2}\right)$ and $\mathcal{C}(f)$ are isometric whereas in case (ii), $\mathcal{C}\left(f_{1}\right)$ and $\mathcal{C}(f)$ are isometric. From this observation and Lemma 1.11 the claimed results follow.

\section{Relative torsion and its Witten deformation}

In subsection 2.1 we introduce the relative torsion (cf [CMM]). As already mentioned in the introduction, the relative torsion cannot be defined as the torsion of the mapping cone associated to the integration map, denoted by Int, as Int cannot be extended to a closed morphism on $L_{2}(\Lambda(M ; \mathcal{E}))$. To circumvent this difficulty we consider $s>\frac{n}{2}+1$, and the composition

$$
L_{2}(\Lambda(M ; \mathcal{E})) \equiv H_{0}(\Lambda(M ; \mathcal{E})) \stackrel{(\Delta+I d)}{\longrightarrow}{ }^{-s / 2} H_{s}(\Lambda(M ; \mathcal{E})) \stackrel{I n t_{s}}{\rightarrow} \mathcal{C}
$$

where $H_{s}\left(\Lambda^{k}(M ; \mathcal{E})\right)$ denotes the completion of the space $\Omega^{k}(M ; \mathcal{E})$ of smooth $k$-forms with respect to the $s$-Sobolev norm, $\Delta_{k}$ denotes the $k$-Laplacian, Int $t_{s}$ is 
the extension of Int to $H_{s}(\Lambda(M ; \mathcal{E}))$ and $\mathcal{C}$ denotes the combinatorial complex associated to $\mathcal{E} \rightarrow M, \mu$ and a generalized triangulation $\tau=\left(h, g^{\prime}\right)$. It turns out that the composition $\operatorname{Int}_{s} \cdot(\Delta+I d)^{-s / 2}$ is a morphism of trace class which induces an isomorphism in algebraic cohomology and, therefore, the corresponding mapping cone $\mathcal{C}\left(\operatorname{Int}_{s}(\Delta+I d)^{-s / 2}\right)$ is algebraically acyclic. We show that it admits a torsion, called the relative torsion $\mathcal{R}$ associated to $\mathcal{E} \rightarrow M, g, \mu, \tau$, and that this torsion is independent of $s>\frac{n}{2}+1$. In the case where $\mathcal{E} \rightarrow M$ is of determinant class (cf [BFKM]), one can show that, $\log \mathcal{R}=\log T_{a n}-\log T_{\text {Reid }}$ where $T_{a n}\left[T_{R e i d}\right]$ is the analytic [Reidemeister] torsion.

To prove that $\log \mathcal{R}$ is a local quantity (in Section 4 ), we will use the Witten deformation of the deRham complex, given by the differentials $d_{k}(t):=e^{-t h} d_{k} e^{t h}$. There are different possibilities for defining the corresponding deformation $\mathcal{R}(t)$ of the relative torsion. We chose one for which the variation $\frac{d}{d t} \log \mathcal{R}(t)$ can be computed. By definition, $\mathcal{R}(t)$ is the torsion of the mapping cone associated to

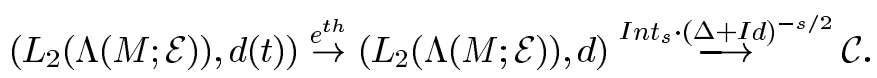

We will show, Theorem 2.1 subsection 2.2 that the variation $\frac{d}{d t} \log \mathcal{R}(t)$ is given by

$$
\frac{d}{d t} \log \mathcal{R}(t)=\operatorname{dim} \mathcal{E}_{x} \int_{M} h e(M, g)
$$

where $e(M, g)$ is the Euler form of the tangent bundle equipped with the LeviCività connection induced from $g$.

\subsection{Relative torsion}

Let $M$ be a closed smooth manifold, $\mathcal{E} \stackrel{\pi}{\rightarrow} M$ a smooth bundle of $\mathcal{A}$-Hilbert modules of finite type equipped with a smooth flat connection $\nabla$, which makes the fiberwise multiplication with elements of $\mathcal{A} \nabla$-parallel. Unlike in [BFKM], or [BFK1] we do not restrict to the case where the Hermitian structure $\mu$ provided by the scalar product $\mu_{x}$ of the fiber $\mathcal{E}_{x}$ of $\mathcal{E}(x \in M)$ is $\nabla$-parallel.

We denote by $\mathcal{E}^{\sharp} \rightarrow M$ the dual bundle of $\mathcal{E} \rightarrow M$. This is a bundle of $\mathcal{A}$-Hilbert modules of finite type with fiber $\mathcal{E}_{x}^{\sharp}$, the Hilbert space dual to $\mathcal{E}_{x}$.

The flat connection $\nabla=\nabla_{\mathcal{E}}$ induces a flat connection $\nabla^{\sharp}:=\nabla_{\mathcal{E}^{\sharp}} \cdot \mathcal{E} \rightarrow M$ and $\mathcal{E}^{\sharp} \rightarrow M$ are canonically isometric as bundles by an $\mathcal{A}$-linear isometry, but this isometry does not intertwine $\nabla$ and $\nabla^{\sharp}$ unless the Hermitian structure $\mu$ is $\nabla_{\mathcal{E}}$-parallel. If $\mathcal{E} \rightarrow M, \nabla$ is induced by the representation $\rho$ of $\Gamma=\pi_{1}\left(M, x_{0}\right)$ then $\mathcal{E}^{\sharp} \rightarrow M, \nabla^{\sharp}$ is induced by $\rho^{\sharp}$. Each of these connections induces a covariant differentiation, 


$$
d_{k} \equiv d_{k, \nabla}: \Omega^{k}(M ; \mathcal{E}) \rightarrow \Omega^{k+1}(M ; \mathcal{E})
$$

and

$$
d_{k}^{\sharp} \equiv d_{k, \nabla^{\sharp}}: \Omega^{k}\left(M ; \mathcal{E}^{\sharp}\right) \rightarrow \Omega^{k+1}\left(M ; \mathcal{E}^{\sharp}\right)
$$

where $\Omega^{k}(M ; \mathcal{E}):=C^{\infty}\left(\Lambda^{k}\left(T^{*} M\right) \otimes \mathcal{E}\right)$ and where $\Omega^{k}\left(M ; \mathcal{E}^{\sharp}\right)$ is defined similarly. The isomorphism $\theta$ induces a canonical isomorphism, again denoted by $\theta$, between $\Omega^{k}(M ; \mathcal{E})$ and $\Omega^{k}\left(M ; \mathcal{E}^{\sharp}\right)$. To simplify notation, we write $\Lambda^{k}(M ; \mathcal{E})$ for the $\mathcal{A}$-Hilbert module of finite type, $\Lambda^{k}(M ; \mathcal{E}):=\Lambda^{k}\left(T^{*} M\right) \otimes \mathcal{E}$.

Given a Riemannian metric $g$ on $M$, let $\mathcal{J}_{k, \mathcal{E}}: \Lambda^{k}(M ; \mathcal{E}) \rightarrow \Lambda^{n-k}\left(M ; \mathcal{E}^{\sharp}\right)$ (with $n=\operatorname{dim} M$ ) be the morphism of $\mathcal{A}$-Hilbert modules defined by $\mathcal{J}_{k, \mathcal{E}}:=\mathcal{J}_{k} \otimes \theta$ where $\mathcal{J}_{k}: \Lambda^{k}\left(T^{*} M\right) \rightarrow \Lambda^{n-k}\left(T^{*} M\right)$ is the Hodge-star operator induced by $g$.

Denote by $d_{k}^{*}: \Omega^{k+1}(M ; \mathcal{E}) \rightarrow \Omega^{k}(M ; \mathcal{E})$ the operator defined by the composition

$$
d_{k}^{*} \equiv d_{k, \nabla}^{*}:=(-1)^{n k-1} \mathcal{J}_{n-k, \mathcal{E}} \circ d_{n-k-1 ; \nabla^{\sharp}} \circ \mathcal{J}_{k+1, \mathcal{E}} .
$$

Further we define a scalar product $\ll \cdot, \cdot \gg: \Omega^{k}(M ; \mathcal{E}) \times \Omega^{k}(M ; \mathcal{E}) \rightarrow \mathbb{C}$ given by

$$
\ll w_{1}, w_{2} \gg:=\int_{M} w_{1} \wedge_{\mathcal{E}} * w_{2} d \operatorname{vol}_{g}
$$

where $* w_{2} \equiv \mathcal{J}_{k, \mathcal{E}} w_{2}$ and $\wedge_{\mathcal{E}}$ is defined by

$$
\begin{aligned}
\Omega^{k}(M ; \mathcal{E}) \times & \Omega^{k}(M ; \mathcal{E}) \stackrel{I d \times \mathcal{J}_{k, \mathcal{E}}}{\rightarrow} \Omega^{k}(M ; \mathcal{E}) \times \Omega^{n-k}\left(M ; \mathcal{E}^{\sharp}\right) \\
& \wedge \Omega^{n}\left(M, \mathcal{E} \otimes \mathcal{E}^{\sharp}\right) \stackrel{e \vartheta}{\rightarrow} \Omega^{n}(M ; \mathbb{C}), \\
\left(w_{1}, w_{2}\right) & \mapsto\left(w_{1}, * w_{2}\right) \mapsto w_{1} \wedge * w_{2} \mapsto w_{1} \wedge \mathcal{E} * w_{2}
\end{aligned}
$$

and $e v$ is the map induced by the evaluation map $\mathcal{E}_{x} \otimes \mathcal{E}_{x}^{\sharp} \rightarrow \mathbb{C}$. Notice that with respect to the inner product $\ll \cdot, \cdot \gg, d_{k}^{*}$ is the formal adjoint of $d_{k}$, i.e. for $w_{1} \in \Omega^{k}(M, \mathcal{E}), w_{2} \in \Omega^{k+1}(M ; \mathcal{E})$

$$
\ll w_{1}, d_{k}^{*} w_{2} \gg=\ll d_{k} w_{1}, w_{2} \gg .
$$

Introduce the Laplacians

$$
\Delta_{k}=d_{k}^{*} d_{k}+d_{k-1} d_{k-1}^{*}: \Omega^{k}(M ; \mathcal{E}) \rightarrow \Omega^{k}(M ; \mathcal{E})
$$


which are second order, elliptic essentially selfadjoint nonnegative $\mathcal{A}$-linear operators. In particular, as $M$ is closed, $\left(I d+\Delta_{k}\right): \Omega^{k}(M ; \mathcal{E}) \rightarrow \Omega^{k}(M ; \mathcal{E})$ is an isomorphism of Fréchet spaces. Using functional calculus, one can define the powers $\left(I d+\Delta_{k}\right)^{s}(s \in \mathbb{R}$ arbitrary). They can be used to define a family of scalar products on $\Omega^{k}(M, \mathcal{E})$ by setting

$$
\ll w_{1}, w_{2} \gg_{s}:=\ll\left(I d+\Delta_{k}\right)^{s / 2} w_{1},\left(I d+\Delta_{k}\right)^{s / 2} w_{2} \gg .
$$

Clearly, $\ll \cdot, \cdot \gg_{s}$ depends on the Hermitian structure $\mu$ and the Riemannian metric $g$. As $d_{k} \Delta_{k}=\Delta_{k+1} d_{k}$ and $\Delta_{k} d_{k}^{*}=d_{k}^{*} \Delta_{k+1}, d_{k}^{*}$ is also the adjoint of $d_{k}$ with respect to the inner product $\ll \cdot, \cdot \gg_{s}$. Denote by $H_{s}\left(\Lambda^{k}(M ; \mathcal{E})\right)$ the completion of $\Omega^{k}(M ; \mathcal{E})$ with respect to the norm induced by the scalar product $\ll \cdot, \cdot \gg_{s}$. As $M$ is supposed to be closed, one obtains a family of complexes

$$
0 \rightarrow \ldots \rightarrow H_{s}\left(\Lambda^{k}(M ; \mathcal{E})\right) \stackrel{d_{k}}{\rightarrow} H_{s}\left(\Lambda^{k+1}(M ; \mathcal{E})\right) \rightarrow \ldots \rightarrow 0
$$

which we denote by $H_{s}(\Lambda(M ; \mathcal{E})) \equiv\left(H_{s}(\Lambda(M ; \mathcal{E})), d\right)$. Here $d_{k}: H_{s}\left(\Lambda^{k}(M ; \mathcal{E})\right)$ $\rightarrow H_{s}\left(\Lambda^{k+1}(M ; \mathcal{E})\right)$ is the maximal closed extension of

$$
d_{k}: \Omega^{k}(M ; \mathcal{E}) \rightarrow \Omega^{k+1}(M ; \mathcal{E}),
$$

its domain is $H_{s+1}\left(\Lambda^{k}(M ; \mathcal{E})\right)$.

As mentioned in Examples $1.6(4), H_{s}(\Lambda(M, \mathcal{E}))$ are $\zeta$-regular complexes of $\mathrm{sF}$ type and the morphisms

$$
\left(1+\Delta_{k}\right)^{t / 2}: H_{s}\left(\Lambda^{k}(M ; \mathcal{E})\right) \rightarrow H_{s-t}\left(\Lambda^{k}(M ; \mathcal{E})\right)
$$

establish an isometry between the complexes $H_{s}(\Lambda(M ; \mathcal{E}))$ and $H_{s-t}(\Lambda(M ; \mathcal{E}))$. The adjoint of $d_{k}$ with respect to the $L_{2}$-inner product, $d_{k}^{*}: H_{s}\left(\Lambda^{k+1}(M ; \mathcal{E})\right) \rightarrow$ $H_{s}\left(\Lambda^{k}(M ; \mathcal{E})\right)$, is given by the maximal closed extension of $d_{k}^{*}: \Omega^{k+1}(M ; \mathcal{E}) \rightarrow$ $\Omega^{k}(M ; \mathcal{E})$ (which depends on $s$ ).

Consider a generalized triangulation $\tau=\left(h, g^{\prime}\right)$ of $M$ (cf introduction of [BFK], also [BFKM]) where $h: M \rightarrow \mathbb{R}$ is a Morse function, $g^{\prime}$ is a compatible Riemannian metric on $M$ and $\mathcal{O}_{h}$ is a collection of orientations of the unstable manifolds defined by $-\operatorname{grad}_{\tilde{g}^{\prime}} \tilde{\mathrm{h}}$, compatible with the deck transformations, where $\tilde{h}$ and $\tilde{g}^{\prime}$ are lifts of $h$ respectively $g^{\prime}$ to the universal cover of $M$ and denote by $\mathcal{C} \equiv(\mathcal{C}(M, \tau, \mathcal{T}), \delta)$ the finite dimensional cochain complex of $\mathcal{A}$-Hilbert modules associated with $\tau$ and $\mathcal{T}:=(\mathcal{E}, \nabla, \mu)$. The $\mathcal{A}$-linear map Int $_{k}: \Omega^{k}(M ; \mathcal{E}) \rightarrow \mathcal{C}^{k}(M, \tau, \mathcal{T})$ provided by integration (cf. [BFKM]) are continuous with respect to the Fréchet topology on $\Omega^{k}(M ; \mathcal{E})$ and extend, for $s>n / 2$, to bounded maps

$$
\operatorname{Int}_{k ; s}: H_{s}\left(\Lambda^{k}(M ; \mathcal{E})\right) \rightarrow \mathcal{C}^{k}(M, \tau, \mathcal{T}) .
$$


Since the integration map intertwines $d$ and $\delta$, they induce morphisms

$$
\operatorname{Int}_{k ; s}: H_{s}\left(\Lambda^{k}(M ; \mathcal{E})\right) \rightarrow \mathcal{C}^{k}(M, \tau, \mathcal{T}) .
$$

As $\mathcal{C}$ is a complex of finite type Hilbert modules and $\operatorname{Int}_{k ; s}$ is bounded for $s>\frac{n}{2}$, Int $_{k ; s}$ is of trace class (cf Examples $1.6(2)$ ). For $s^{\prime} \leq s$, denote by $I n_{* ; s, s^{\prime}}$ the embedding

$$
\operatorname{In}_{k ; s, s^{\prime}}: H_{s}\left(\Lambda^{k}(M ; \mathcal{E})\right) \hookrightarrow H_{s^{\prime}}\left(\Lambda^{k}(M ; \mathcal{E})\right)
$$

which is a morphism of cochain complexes. For $s-s^{\prime}>n, I n_{k ; s, s^{\prime}}$ is of trace class.

Proposition 2.1 (cf [CMM, section 4])

The following families of morphisms induce isomorphisms in algebraic cohomology:

(i) $(I d+\Delta)^{s / 2}: H_{r}(\Lambda(M ; \mathcal{E})) \rightarrow H_{r-s}(\Lambda(M ; \mathcal{E})) \quad(\forall r, s)$;

(ii) $\operatorname{In}_{s, s^{\prime}}: H_{s}(\Lambda(M ; \mathcal{E})) \rightarrow H_{s^{\prime}}(\Lambda(M ; \mathcal{E})) \quad\left(s \geq s^{\prime}\right)$;

(iii) Int $_{s}: H_{s}(\Lambda(M ; \mathcal{E})) \rightarrow \mathcal{C}(M, \tau, \mathcal{T})(s>n / 2)$.

Proof (i) is obvious since $(I d+\Delta)^{s / 2}$ is an isometric morphism.

(ii) For ease of notation, let $H_{k ; s}:=H_{s}\left(\Lambda^{k}(M ; \mathcal{E})\right)$. Denote by $Q_{k}: H_{k ; 0} \rightarrow H_{k ; 0}$ the $L_{2}$-orthogonal spectral projector corresponding to the interval $[0, r], r>0$ and consider its restriction to $H_{k ; s}, s>0$. Due to the ellipticity of $\Delta_{k}, Q_{k} \omega \in$ $\Omega^{k}(M ; \mathcal{E})$ and $Q_{k}\left(H_{k ; s}\right)$ is isomorphic to $Q_{k}\left(\Omega^{k}(M ; \mathcal{E})\right)$. As $H_{k, s}=Q_{k}\left(H_{k ; s}\right) \oplus$ $\left(I d-Q_{k}\right)\left(H_{k ; s}\right), H_{s}(\Lambda(M ; \mathcal{E}))$ is the sum of two subcomplexes, $\mathcal{C}^{1} \oplus \mathcal{C}^{2}$ where $\mathcal{C}_{k}^{1}:=Q_{k}\left(H_{k ; s}\right)$ and $\mathcal{C}_{k}^{2}:=\left(I d-Q_{k}\right)\left(H_{k, s}\right)$. Notice that $\mathcal{C}^{2}$ is algebraically acyclic by remark after $(1.20) . \mathcal{C}^{1}$ has the same algebraic cohomology as $H_{s}(\Lambda(M, \mathcal{E}))$ and that $I n_{k ; s, s^{\prime}}=\left(\begin{array}{l|l}I d & 0 \\ \hline 0 & \left.I n_{k ; s, s^{\prime}}\right|_{\mathcal{C}_{k}^{2}}\end{array}\right)$. Therefore, $I n_{s, s^{\prime}}$, induces an isomorphism in algebraic cohomology.

(iii) We use Witten's deformation of the deRham complex (cf [BFK2, section 3], also [BFKM, section 5]). For $t \in \mathbb{R}$, let $d_{k}(t):=e^{-t h} d_{k} e^{t h}$ be the deformed exterior differential where $h: M \rightarrow \mathbb{R}$ is the Morse function of a generalized triangulation $\tau=\left(h, g^{\prime}\right)$ ( $\mathrm{h}$ not necessarily selfindexing). For $t$ sufficiently large, the spectrum of the deformed Laplacian $\Delta_{k}(t)$ splits into a small part contained in the interval $[0,1]$ and a large part, contained in an interval of the form $[C t, \infty]$ with $C>0$. Denote by $\left(\Omega_{s m}(M ; \mathcal{E})(t), d(t)\right)$ 
the subcomplex associated to the small part of the spectrum. In particular, $\Omega_{s m}^{k}(t) \equiv \Omega_{s m}^{k}(M ; \mathcal{E})(t)=Q_{k}(t)\left(H_{k ; s}\right)$ where $Q_{k}(t)$ denotes the orthogonal spectral projector of $\Delta_{k}(t)$ corresponding to the interval [0,1]. As $\Delta_{k}(t)$ is elliptic, one concludes that $\Omega_{s m}^{k}(t) \equiv \Omega_{s m}^{k}(M ; \mathcal{E})(t) \subseteq \Omega^{k}(M ; \mathcal{E})$ and, by [BFK2, section 3] (cf also [BFKM, section 5]), is an $\mathcal{A}$-Hilbert module of finite type. Similarly as in the proof of (ii), $H_{s}(\Lambda(M ; \mathcal{E}))$ is the sum of two subcomplexes, $\Omega_{s m}(t) \oplus H_{s, l a}(t)$, where $H_{k ; s, l a}:=\left(I d-Q_{k}(t)\right)\left(H_{k ; s}\right)$. Introduce

$$
f_{k}(t): \Omega_{s m}^{k}(M ; \mathcal{E})(t) \rightarrow \mathcal{C}^{k}(M, \tau, \mathcal{T})
$$

given by $f_{k}(t):=\operatorname{Int}_{k} e^{t h}$. One verifies that $f(t):\left(\Omega_{s m}(t), d(t)\right) \rightarrow(\mathcal{C}, \delta)$ is a morphism of cochain complexes. By the Helffer-Sjöstrand theory (cf [BFK2, section 3], also [BFKM, section 5]), $f(t)$ is an isomorphism for $t$ sufficiently large. In particular, it induces an isomorphism in algebraic cohomology. For $t$ sufficiently large, $H_{s}(\Lambda$ $(M ; \mathcal{E}))$ can be decomposed into two subcomplexes, $\mathcal{C}^{1} \oplus \mathcal{C}^{2}$ where $\mathcal{C}^{1}=\left(e^{\text {th }} \Omega_{s m}\right.$ $(t), d)$ and $\mathcal{C}^{2}=\left(e^{t h} H_{s, l a}(t), d\right)$. Notice that $\mathcal{C}^{2}$ is algebraically acyclic and that

$\mathcal{C}^{1}$ has the same algebraic cohomology as $H_{s}(\Lambda(M ; \mathcal{E}))$. For $s>n / 2$,

$$
\text { Int }_{s}: H_{s}(\Lambda(M ; \mathcal{E}))=\mathcal{C}^{1} \oplus \mathcal{C}^{2} \rightarrow \mathcal{C}
$$

is well defined and takes the form $\left(f(t) e^{-t h},\left.\operatorname{Int}_{s}\right|_{\mathcal{C}^{2}}\right)$. Therefore $I n t_{s}$ induces an isomorphism in algebraic cohomology.

Remark 2.2 For $s-s^{\prime}>n+1$ the inclusion In $_{s, s^{\prime}}$ is a morphism of trace class (cf Definition 1.9).

As $H_{s}(\Lambda(M ; \mathcal{E}))$ is a $\zeta$-regular complex of $\mathrm{sF}$ type (for any $s \in \mathbb{R}$ ), we conclude from Proposition 2.1 and Lemma 1.12 that the mapping cone $\mathcal{C}\left(\operatorname{In}_{s, s^{\prime}}\right)$ is algebraically acyclic and thus has a well defined torsion $T\left(\mathcal{C}\left(\operatorname{In}_{s, s^{\prime}}\right)\right)\left(s-s^{\prime}>n+1\right)$.

Proposition 2.3 For $s-s^{\prime}>n+1, \log T\left(\mathcal{C}\left(\operatorname{In}_{s, s^{\prime}}\right)\right)=0$.

Proof Recall that the mapping cone $\mathcal{C}\left(I n_{s, s^{\prime}}\right)$ is given by

$$
\begin{gathered}
\mathcal{C}\left(\operatorname{In}_{s, s^{\prime}}\right)_{k}:=H_{s^{\prime}}\left(\Lambda^{k-1}(M ; \mathcal{E})\right) \oplus H_{s}\left(\Lambda^{k}(M ; \mathcal{E})\right) ; \\
d\left(\operatorname{In}_{s, s^{\prime}}\right)_{k}=\left(\begin{array}{cc}
-d_{k-1} & \operatorname{In}_{k ; s, s^{\prime}} \\
0 & d_{k}
\end{array}\right) .
\end{gathered}
$$

Therefore, by (1.23) and (2.4), 


$$
\Delta\left(I_{s, s^{\prime}}\right)_{k}=\left(\begin{array}{cc}
\Delta_{k-1}+\left(I d+\Delta_{k-1}\right)^{s^{\prime}-s} & 0 \\
0 & \Delta_{k}+\left(I d+\Delta_{k}\right)^{s^{\prime}-s}
\end{array}\right)
$$

Proposition 2.3 then follows from Lemma 2.4 below.

Lemma 2.4 Assume that $(\mathcal{C}, \delta)$ is a $\zeta$-regular cochain complex of sF type. Then

(i) $\sum_{k}(-1)^{k} \log \operatorname{det}\left(\Delta_{k}+I d\right)=0$;

(ii) $\sum_{k}(-1)^{k} \log \operatorname{det}\left(\Delta_{k}+\left(I d+\Delta_{k}\right)^{s^{\prime}-s}\right)=0$.

Proof As the two statements can be proved in the same way, we consider only the second one. With respect to the Hodge decomposition, $\Delta_{k}+I d$ takes the form (cf (1.18))

$$
\begin{aligned}
& \Delta_{k}+\left(I d+\Delta_{k}\right)^{s^{\prime}-s}= \\
& =\operatorname{diag}\left(I d, \underline{\Delta}_{k}^{+}+\left(I d+\underline{\Delta}_{k}^{+}\right)^{s^{\prime}-s}, \underline{\Delta}_{k}^{-}+\left(I d+\underline{\Delta}_{k}^{-}\right)^{s^{\prime}-s}\right) .
\end{aligned}
$$

where $\underline{\Delta}_{k}^{+}:=\underline{d}_{k-1} \underline{d}_{k-1}^{*}$ and $\underline{\Delta}_{k}^{-}:=\underline{d}_{k}^{*} \underline{d}_{k}$ are $\zeta$-regular operators of sF type and

$\log \operatorname{det}\left(\Delta_{k}+\left(I d+\Delta_{k}\right)^{s^{\prime}-s}\right)=\log \operatorname{det}\left(\underline{\Delta}_{k}^{+}+\left(I d+\underline{\Delta}_{k}^{+}\right)^{s^{\prime}-s}\right)+$

$$
+\log \operatorname{det}\left(\underline{\Delta}_{k}^{-}++\left(I d+\underline{\Delta}_{k}^{-}\right)^{s^{\prime}-s}\right) \text {. }
$$

As $\underline{\Delta}_{k}^{+}$and $\underline{\Delta}_{k-1}^{-}$have the same spectral distribution function we conclude that

$$
\log \operatorname{det}\left(\underline{\Delta}_{k}^{+}+\left(I d+\underline{\Delta}_{k}^{+}\right)^{s^{\prime}-s}\right)=\log \operatorname{det}\left(\underline{\Delta}_{k-1}^{-}+\left(I d+\underline{\Delta}_{k-1}^{-}\right)^{s^{\prime}-s}\right) .
$$

This proves the claimed statement.

Proposition 2.5 Let $s>\frac{n}{2}+1, \tilde{s}>s+n+1$ and $p \in \mathbb{R}_{>0}$. Then

(i) $\operatorname{Int}_{s}: H_{s}(\Lambda(M ; \mathcal{E})) \rightarrow \mathcal{C}(M, \tau, \mathcal{T})$

is a morphism of trace class which induces an isomorphism in algebraic cohomology. Moreover

$$
\log T\left(\mathcal{C}\left(\text { Int }_{\tilde{s}}\right)\right)=\log T\left(\mathcal{C}\left(\text { Int }_{s}\right)\right)
$$

and

$$
\log T\left(\mathcal{C}\left(\text { Int }_{s}\right)\right)=\log T\left(\mathcal{C}\left(\operatorname{Int}_{s}(\Delta+\operatorname{Id})^{p / 2}\right)\right),
$$

with $(\Delta+I d)^{p / 2}: H_{s+p}(\Lambda(M ; \mathcal{E})) \rightarrow H_{s}(\Lambda(M ; \mathcal{E}))$ 
Proof By Proposition 2.1, Int $t_{s}$ is a morphism of cochain complexes which induces an isomorphism in algebraic cohomology. We claim that Int $_{s}$ is a morphism of trace class (cf Definition 1.10). As we have already observed, Int $k ; s$ is an operator of trace class for $s>n / 2$ as it is bounded and $\mathcal{C}^{k}$ is a Hilbert module of finite type. By the same reason, $\delta_{k}^{*} \operatorname{Int}_{k+1 ; s}$ is of trace class for $s>n / 2$. Finally we have to verify that $\operatorname{Int}_{k ; s} d_{k}^{*}$ is of trace class. Use that Int $_{k ; s}=\operatorname{Int}_{k ; s-1} \operatorname{In} n_{k ; s, s-1}$ and that $I n_{k ; s, s-1} d_{k}^{*}$ is a bounded operator to deduce from Proposition 1.3 that $\operatorname{Int}_{k ; s} d_{k}^{*}$ is of trace class for $s>\frac{n}{2}+1$. Further notice that $I n t_{\tilde{s}}=I n t_{s} \cdot I n_{\tilde{s}, s}$ for arbitrary $\tilde{s}>s+n+1$ where $\operatorname{In}_{\tilde{s}, s}$ is a morphism of trace class (Remark 2.2) which induces an isomorphism in algebraic cohomology (Proposition 2.1). From Proposition 1.15 and Proposition 2.3 we then conclude that

$$
\begin{aligned}
\log T\left(\mathcal{C}\left(\operatorname{Int}_{\tilde{s}}\right)\right) & =\log T\left(\mathcal{C}\left(\operatorname{In}_{\tilde{s}, s}\right)\right)+\log T\left(\mathcal{C}\left(\operatorname{Int}_{s}\right)\right) \\
& =\log T\left(\mathcal{C}\left(\operatorname{Int}_{s}\right)\right) .
\end{aligned}
$$

It follows from Proposition 1.3 and the fact that $(\Delta+I d)^{p / 2}$ is an isometry of cochain complexes that $\operatorname{Int}_{s}(\Delta+I d)^{p / 2}$ is a morphism of trace class which induces an isomorphism in algebraic cohomology. Applying Lemma 1.11 and Proposition 1.16 yields formula (2.13).

We are now ready to define the relative torsion. Our definition differs slightly from the one first introduced by [CMM].

Given $M, \mathcal{T}, g, \tau$, define $\mathcal{R}_{s}=\mathcal{R}_{s}(M, \mathcal{T}, g, \tau)$,

$$
\log \mathcal{R}_{s}:=\log T\left(\mathcal{C}\left(\operatorname{Int}_{s}(\Delta+I d)^{-s / 2}\right)\right) \quad(s>n / 2+1) .
$$

Notice that, by Proposition 2.5, $\log \mathcal{R}_{s}=\log T\left(\mathcal{C}\left(\right.\right.$ Int $\left.\left._{s}\right)\right)$ and $\mathcal{R}_{s}$ is independent of $s>\frac{n}{2}+1$.

Definition 2.6 The relative torsion is defined by $\mathcal{R}:=\mathcal{R}_{s}(M, \mathcal{T}, g, \tau)$.

The name of relative torsion is justified by the observation, due to [CMM], that in the case where $\mathcal{E} \rightarrow M$ is of determinant class - and therefore, the analytic torsion $T_{a n}$ and the Reidemeister torsion $T_{\text {Reid }}$ are well defined (cf [BFKM]) $\mathcal{R}$ is given by

$$
\log \mathcal{R}=\log T_{\text {an }}-\log T_{\text {Reid }}
$$

To see that (2.15) hold we would like to apply Proposition 1.10 (Milnor's Lemma). However $H_{s}(\Lambda(M ; \xi))$ is not a complex of finite dimension, we therefore decompose the mapping cone $\mathcal{C}\left(\operatorname{Int}_{s}\right)$ as follows: As shown in the proof of Proposition 2.1 (iii), $H_{s}(\Lambda(M ; \mathcal{E}))=\mathcal{C}^{1} \oplus \mathcal{C}^{2}$ and $f:\left.\operatorname{Int}_{s}\right|_{\mathcal{C}^{1}}: \mathcal{C}^{1} \rightarrow \mathcal{C}^{2}$ 
is a bijective morphism where $\mathcal{C}^{2}$ is algebraically acyclic and $\mathcal{C}^{1}$ is given by $\mathcal{C}^{1}:=\left(e^{t h} \Omega_{s m}(t), d\right)$. Notice that $\mathcal{C}^{1}$ has the same algebraic cohomology as $H_{s}(\Lambda(M ; \mathcal{E}))$. Denote by $\mathcal{C}^{3}$ the mapping cone $\mathcal{C}(f)$. As $f$ is a bijective morphism, $\mathcal{C}^{3}=\mathcal{C}(f)$ is algebraically acyclic. As $f$ satisfies the assumption of Lemma 1.14 one concludes that

$$
\log \mathcal{R}=\log T\left(\mathcal{C}\left(\text { Int }_{s}\right)\right)=\log T\left(\mathcal{C}^{3}\right)+\log T\left(\mathcal{C}^{2}\right) .
$$

The term $\log T\left(\mathcal{C}^{3}\right)$ has to be analyzed further. By assumption, $(M, \rho)$ is of determinant class. Therefore $\mathcal{C}^{1}$ is of determinant class as well. We then obtain the following short exact sequence of cochain complexes, each of them being of determinant class,

$$
0 \rightarrow \Sigma \mathcal{C} \rightarrow \mathcal{C}^{3} \rightarrow \mathcal{C}^{1} \rightarrow 0
$$

As $\Sigma \mathcal{C}, \mathcal{C}^{3}$ and $\mathcal{C}^{1}$ are of finite dimension we can apply Proposition 1.10 to conclude that

$$
\log T\left(\mathcal{C}^{3}\right)=\log T(\Sigma \mathcal{C})+\log T\left(\mathcal{C}^{1}\right)+\log T(\mathcal{H})
$$

where $\mathcal{H}$ denotes the long weakly exact sequence in reduced cohomology and is of determinant class. By Lemma 1.14 and the definition of the combinatorial torsion $T_{\text {comb }}(\mathrm{cf}[\mathrm{BFKM}]), \log T(\Sigma \mathcal{C})=-\log T(\mathcal{C})=-\log T_{\text {comb }}$. By the definition of the analytic torsion (cf $[\mathrm{BFKM}]), \log T\left(\mathcal{C}^{1}\right)+\log T\left(\mathcal{C}^{2}\right)=\log T_{a n}$. Further $\mathcal{H}$ is given by

$$
\ldots \rightarrow \bar{H}^{i}(\Sigma \mathcal{C}) \rightarrow \bar{H}^{i}\left(\mathcal{C}^{3}\right) \rightarrow \bar{H}^{i}\left(\mathcal{C}^{1}\right) \stackrel{\bar{H}\left(\delta_{i}\right)}{\rightarrow} \bar{H}^{i+1}(\Sigma \mathcal{C}) \rightarrow \ldots
$$

Recall that $\mathcal{C}^{3}$ is algebraically acyclic and $\bar{H}\left(\delta_{i}\right): \bar{H}^{i}\left(\mathcal{C}^{1}\right) \rightarrow \bar{H}^{i}(\mathcal{C})$ is given by the restriction of $\operatorname{Int}_{i ; s}$ to $\mathcal{H}_{i}=\operatorname{ker}\left(\Delta_{i}\right)$ and, by the definition of the metric part $T_{m e t}$ of the torsion

$$
\log T(\mathcal{H})=-\log T_{\text {met }} .
$$

Combining the various equalities above leads to (2.15).

\subsection{Witten deformation of the relative torsion}

To obtain a local formula for $\log \mathcal{R}$ we consider the deformed relative torsion $\mathcal{R}(t)$ obtained by considering the Witten deformation of the deRham complex.

Using the generalized triangulation $\tau=\left(h, g^{\prime}, \mathcal{O}_{h}\right)$ we consider for $s>n / 2+1$ the following composition $g_{s}(t)$ of morphisms.

$$
\left(L_{2}(\Lambda(M ; \mathcal{E})), d(t)\right) \stackrel{e^{t h}}{\longrightarrow}\left(L_{2}(\Lambda(M ; \mathcal{E})), d\right) \stackrel{(\Delta+I d)^{-s / 2}}{\rightarrow}\left(H_{s}(\Lambda(M, \mathcal{E})), d\right)
$$




$$
\stackrel{\operatorname{Int} s}{\rightarrow}(\mathcal{C}, \delta)
$$

where we recall that $d_{k}(t)=e^{-t h} d_{k} e^{t h}$. For $s>n / 2+1, I n t_{s}$ is a morphism of trace class (cf Proposition 2.5) and thus, by Proposition 1.3, the composition $g_{s}(t)$ is a morphism of trace class. Due to Proposition 2.1, $g_{s}(t)$ induces an isomorphism in algebraic cohomology, hence $\log T\left(\mathcal{C}\left(g_{s}(t)\right)\right)$ is well defined.

Proposition 2.7 For $s+p>s>2 n+2$ and $t \in \mathbb{R}$,

$$
\log T\left(\mathcal{C}\left(g_{s}(t)\right)\right)=\log T\left(\mathcal{C}\left(g_{s+p}(t)\right)\right)
$$

Proof Using that Int $t_{s}=\operatorname{Int}_{s / 2} I n_{s, s / 2}, g_{s}(t)$ is given by $g_{s}(t)=\operatorname{Int}_{s / 2} \alpha_{s}(t)$ where $\alpha_{s}(t):=I n_{s, s / 2}(\Delta+I d)^{-s / 2} e^{t h}$. As $s>2 n+2, \alpha_{s}(t)$ (cf. Remark 2.2) and Int $_{s / 2}$ (cf. Proposition 2.5) are morphisms of trace class. By Proposition 2.1, both Int $_{s / 2}$ and $\alpha_{s}(t)$ induce isomorphisms in algebraic cohomology. Therefore we can apply Proposition 1.16 to conclude that

$$
\log T\left(\mathcal{C}\left(g_{s}(t)\right)\right)=\log T\left(\mathcal{C}\left(\operatorname{Int}_{s / 2}\right)\right)+\log T\left(\mathcal{C}\left(\alpha_{s}(t)\right)\right) .
$$

Observe that, $I n_{s+p, \frac{s}{2}+p}(\Delta+I d)^{-\frac{s+p}{2}}=(\Delta+I d)^{-p / 2} I n_{s, s / 2}(\Delta+I d)^{-s / 2}$ to deduce that

$$
g_{s+p}(t)=\operatorname{Int}_{p+\frac{s}{2}}(\Delta+I d)^{-p / 2} \alpha_{s}(t) .
$$

Using the same arguments as above we can apply Proposition 1.16 to obtain

$$
\begin{aligned}
\log T\left(\mathcal{C}\left(g_{s+p}\right)\right)= & \log T\left(\mathcal{C}\left(\operatorname{Int}_{p+s / 2} \cdot(\Delta+I d)^{-p / 2}\right)\right)+ \\
& +\log T\left(\mathcal{C}\left(\alpha_{s}\right)\right) .
\end{aligned}
$$

By Proposition 2.5,

$$
\log T\left(\mathcal{C}\left(\operatorname{Int}_{\frac{s}{2}+p}\right)\right)=\log T\left(\mathcal{C}\left(\operatorname{Int}_{\frac{s}{2}+p}(\Delta+I d)^{-p / 2}\right)\right)
$$

and (cf (2.14))

$$
\log T\left(\mathcal{C}\left(\operatorname{Int}_{s / 2+p}\right)\right)=\log T\left(\mathcal{C}\left(\operatorname{Int}_{s / 2}\right)\right) .
$$

Combining (2.17)-(2.20) leads to the claimed result.

In view of Proposition 2.7, $\log T\left(\mathcal{C}\left(g_{s}(t)\right)\right)$ is independent of $s$. 
Definition 2.8 The Witten deformation $\mathcal{R}(t)$ of the relative torsion is defined by

$$
\log \mathcal{R}(t):=\log T\left(\mathcal{C}\left(g_{s}(t)\right)\right)(s>n+2)
$$

Using the same arguments as for the verification of (2.15) one sees that, for $\mathcal{E} \rightarrow M$ of determinant class,

$$
\log \mathcal{R}(t)=\log T_{a n}(t)-\log T_{\text {comb }}-\log T_{\text {met }}(t)
$$

where $T_{a n}(t)$ denotes the analytic torsion of the deformed deRham complex (cf $[\mathrm{BFKM}]), T_{\text {comb }}$ denotes again the combinatorial torsion and $\log T_{\text {met }}(t)=$ $-\log T(\mathcal{H}(t))$ is the torsion of the long weakly exact sequence in cohomology obtained in a similar fashion as in the case of (2.15).

Our next objective is to calculate the variation for $\log \mathcal{R}(t)$ :

\section{Theorem 2.1}

$$
\frac{d}{d t} \log \mathcal{R}(t)=\operatorname{dim} \mathcal{E}_{x} \int_{M} h \cdot e(M, g)
$$

where $e(M, g)$ is the Euler form of the tangent bundle equipped with the LeviCività connection induced from $g$. In particular, $\frac{d}{d t} \log \mathcal{R}(t)$ is a local quantity, independent of $t$ and the Hermitian structure. Moreover if $n=\operatorname{dim} M$ is odd, then

$$
\frac{d}{d t} \log \mathcal{R}(t) \equiv 0
$$

Proof For $s>n+2$, introduce the morphism $g_{s}:=\operatorname{Int}_{s}(\Delta+I d)^{-s / 2}$. Then $g_{s}(t)=g_{s} e^{t h}$. Observe that for all $t$, the mapping cone $\mathcal{G}(t):=\mathcal{C}\left(g_{s}(t)\right)$ is a cochain complex whose $k^{\prime}$ th component $\mathcal{G}_{k}:=\mathcal{C}\left(g_{s}(t)\right)_{k}$ is independent of $t$, given by

$$
\mathcal{G}_{k}=\mathcal{C}^{k-1}(M, \tau, \mathcal{T}) \oplus L_{2}\left(\Lambda^{k}(M ; \mathcal{E})\right)
$$

With respect to the decomposition $(2.22), d_{k}(t) \equiv d_{k}^{\mathcal{G}}(t): \mathcal{G}_{k} \rightarrow \mathcal{G}_{k+1}$ takes the form

$$
d_{k}(t)=E_{k+1}(-t) D_{k} E_{k}(t) ; d_{k}(t)^{*}=E_{k}(t) D_{k}^{*} E_{k+1}(-t) .
$$

where 


$$
E_{k}(t):=\left(\begin{array}{cc}
I d & 0 \\
0 & e^{t h}
\end{array}\right), D_{k}:=\left(\begin{array}{cc}
-\delta_{k-1} & g_{k ; s} \\
0 & d_{k}
\end{array}\right)
$$

According to Proposition 2.5 and 1.12, the mapping cone $\mathcal{C}\left(g_{s}(t)\right)$ is a $\zeta$-regular, algebraically acyclic cochain complex of $\mathrm{sF}$ type. Denote by

$$
\Delta_{k}(t) \equiv \Delta_{k}^{\mathcal{G}}(t) \text { the } k \text {-Laplacian associated to } \mathcal{G} \equiv \mathcal{C}\left(g_{s}(t)\right)
$$

$$
\Delta_{k}(t):=\Delta_{k}^{+}(t)+\Delta_{k}^{-}(t)
$$

where

$$
\Delta_{k}^{+}(t):=d_{k-1}(t) d_{k-1}^{*}(t), \Delta_{k}^{-}(t):=d_{k}^{*}(t) d_{k}(t) .
$$

With respect to the Hodge decomposition $\mathcal{G}_{k}=\mathcal{G}_{k}^{+}(t) \oplus \mathcal{G}_{k}^{-}(t)$,

$$
\mathcal{G}_{k}^{+}(t):=\overline{d_{k-1}(t)\left(\mathcal{G}_{k-1}\right)} ; \mathcal{G}_{k}^{-}(t):=\overline{d_{k}^{*}(t)\left(\mathcal{G}_{k+1}\right)}
$$

$d_{k}(t)$ is of the form $d_{k}(t)=\left(\begin{array}{cc}0 & 0 \\ 0 & \underline{d}_{k}(t)\end{array}\right)$ and thus $\Delta_{k}^{+}(t)=\left(\begin{array}{cc}\Delta_{k}^{+}(t) & 0 \\ 0 & 0\end{array}\right)$ and $\Delta_{k}^{-}(t)=\left(\begin{array}{cc}0 & 0 \\ 0 & \Delta_{k}^{-}(t)\end{array}\right)$ where $\underline{\Delta}_{k}^{+}(t):=\underline{d}_{k-1}(t) \underline{d}_{k-1}^{*}(t)$ and $\underline{\Delta}_{k}^{-}(t):=$ $\underline{d}_{k}^{*}(t) \underline{d}_{k}(t)$. As $\mathcal{C}\left(g_{s}(t)\right)$ is a $\zeta$-regular cochain complex, the operators $\underline{d}_{k}(t)$, according to Definition 1.9, satisfy $\mathrm{Op}(1)-\mathrm{Op}(7)$. As $\mathcal{C}\left(g_{s}(t)\right)$ is algebraically acyclic, they also satisfy $\mathrm{Op}(8)$. (In fact, the operators $\underline{d}_{k}(t)$ satisfy $O p(7)$, because the corresponding heat traces admit an asymptotic expansion of the form (Asy) (cf Lemma 1.1).)

To compute $\frac{d}{d t} \log \mathcal{R}(t)$ we proceed similarly as in [BFKM] where we compute $\frac{d}{d t} \log T_{a n}(t)$. According to Definition 2.8, the relative torsion $\mathcal{R}(t)=T\left(\mathcal{C}\left(g_{s}(t)\right)\right)$ is given by

$$
\log \mathcal{R}(t)=\frac{1}{2} \sum_{k}(-1)^{k+1} \log \operatorname{det} \underline{\Delta}_{k}^{+}(t)
$$

and

$$
\begin{gathered}
\frac{d}{d t} \log \operatorname{det}\left(\underline{\Delta}_{k}^{+}(t)\right)=F \cdot p_{\cdot z=0} \operatorname{Tr}\left(\frac{d}{d t}\left(\underline{\Delta}_{k}^{+}(t)\right)\left(\underline{\Delta}_{k}^{+}(t)\right)^{-z-1}\right) \\
=F \cdot p \cdot z=0 \operatorname{Tr}\left(\frac{d}{d t}\left(E_{k}(-t) D_{k-1} E_{k-1}(2 t) D_{k-1}^{*} E_{k}(-t)\right)\left(\Delta_{k}^{+}(t)\right)^{-z-1}\right) .
\end{gathered}
$$

(By F.p. $z=0$ we denote the constant term in the Laurent expansion at $z=0$ of the expression which follows it.) To simplify writing we suppress momentarily the subscript $k$. Then 
(2.26)

$$
\begin{aligned}
& \frac{d}{d t}\left(E(-t) D E(2 t) D^{*} E(-t)\right)= \\
& =\left(\begin{array}{cc}
0 & 0 \\
0 & -h
\end{array}\right) E(-t) D E(2 t) D^{*} E(-t) \\
& +E(-t) D\left(\begin{array}{cc}
0 & 0 \\
0 & 2 h
\end{array}\right) E(2 t) D^{*} E(-t) \\
& +E(-t) D E(2 t) D^{*} E(-t)\left(\begin{array}{cc}
0 & 0 \\
0 & -h
\end{array}\right) .
\end{aligned}
$$

Substituting (2.26) into (2.25) we obtain

$$
\begin{aligned}
& \frac{d}{d t} \log \operatorname{det}\left(\underline{\Delta}^{+}(t)\right)= \\
& =F \cdot p \cdot z=0 \\
& +F r\left(\left(\begin{array}{cc}
0 & 0 \\
0 & -h
\end{array}\right) d(t) d(t)^{*} \Delta^{+}(t)^{-z-1}\right) \\
& +F \cdot p \cdot\left(d(t)\left(\begin{array}{cc}
0 & 0 \\
0 & 2 h
\end{array}\right) d(t)^{*} \Delta^{+}(t)^{-z-1}\right) \\
& +\operatorname{Tr}\left(d(t) d(t)^{*}\left(\begin{array}{cc}
0 & 0 \\
0 & -h
\end{array}\right) \Delta^{+}(t)^{-z-1}\right) .
\end{aligned}
$$

Using that $d(t) d(t)^{*} \Delta^{+}(t)^{-z-1}=\Delta^{+}(t)^{-z}$ together with the commutativity of the trace we conclude

$$
\begin{aligned}
& \frac{d}{d t} \log \operatorname{det}\left(\underline{\Delta}_{k}^{+}(t)\right)= \\
& =F \cdot p \cdot z=0 \\
& +F r\left(\left(\begin{array}{cc}
0 & 0 \\
0 & -2 h
\end{array}\right) \Delta_{k}^{+}(t)^{-z}\right) \\
& +F \cdot \cdot_{z=0} \operatorname{Tr}\left(\left(\begin{array}{cc}
0 & 0 \\
0 & 2 h
\end{array}\right) d_{k-1}^{*}(t) \Delta_{k}^{+}(t)^{-z-1} d_{k-1}(t)\right) .
\end{aligned}
$$

From $d_{k-1}^{*}(t) \Delta_{k}^{+}(t)=d_{k-1}^{*}(t) d_{k-1}(t) d_{k-1}(t)^{*}=\Delta_{k-1}^{-}(t) d_{k-1}(t)^{*}$ we conclude that

$$
d_{k-1}^{*} \Delta_{k}^{+}(t)^{-z-1} d_{k-1}(t)=\left(\Delta_{k-1}^{-}(t)\right)^{-z-1} \Delta_{k-1}^{-}(t)=\Delta_{k-1}^{-}(t)^{-z}
$$

and therefore, after substituting into (2.28)

$$
\begin{aligned}
& \frac{d}{d t} \log \operatorname{det}\left(\underline{\Delta}_{k}^{+}(t)\right)= \\
& =F \cdot p_{z=0} \operatorname{Tr}\left(\left(\begin{array}{cc}
0 & 0 \\
0 & -2 h
\end{array}\right)\left(\Delta_{k}^{+}(t)^{-z}-\Delta_{k-1}^{-}(t)^{-z}\right)\right) .
\end{aligned}
$$

This leads to

$$
\frac{d}{d t} \log T\left(\mathcal{C}\left(g_{s}(t)\right)\right)=\sum_{k}(-1)^{k} F \cdot p \cdot z=0 \operatorname{Tr}\left(\left(\begin{array}{cc}
0 & 0 \\
0 & h
\end{array}\right) \Delta_{k}(t)^{-z}\right) .
$$


As $\mathcal{C}\left(g_{s}(t)\right)$ is algebraically acyclic, $\Delta_{k}(t)$ has no spectrum near 0 and therefore $\zeta^{\mathrm{II}}(z):=\frac{1}{\Gamma(z)} \int_{1}^{\infty} \lambda^{z-1} \operatorname{Tr}\left(\left(\begin{array}{ll}0 & 0 \\ 0 & h\end{array}\right) e^{-\lambda \Delta_{k}(t)}\right) d \lambda$ is an entire function, with $\zeta^{\mathrm{II}}(0)=0$.

In view of the fact that the heat trace $\operatorname{Tr}\left(\left(\begin{array}{ll}0 & 0 \\ 0 & h\end{array}\right) e^{-\lambda \Delta_{k}(t)}\right)$ admits an asymptotic expansion at $\lambda=0$ of the form (Asy) one obtains (cf Lemma 1.1 and $\mathrm{Op}(7))$

$$
\begin{aligned}
F . p \cdot z=0 & \operatorname{Tr}\left(\left(\begin{array}{cc}
0 & 0 \\
0 & h
\end{array}\right) \Delta_{k}(t)^{-z}\right)= \\
& =F \cdot p \cdot z=0\left(\frac{1}{\Gamma(z)} \int_{0}^{\infty} \lambda^{z-1} \operatorname{Tr}\left(\left(\begin{array}{cc}
0 & 0 \\
0 & h
\end{array}\right) e^{-\lambda \Delta_{k}(t)}\right) d \lambda\right) \\
& =F \cdot p \cdot z=0\left(\frac{1}{\Gamma(z)} \int_{0}^{1} \lambda^{z-1} \operatorname{Tr}\left(\left(\begin{array}{cc}
0 & 0 \\
0 & h
\end{array}\right) e^{-\lambda \Delta_{k}(t)}\right) d \lambda\right) .
\end{aligned}
$$

With respect to the decomposition (2.22) of the mapping cone $\mathcal{C}\left(g_{s}(t)\right)$, the Laplacian $\Delta_{k}(t) \equiv \Delta_{k}^{\mathcal{G}}(t)$ takes the form

$$
\Delta_{k}^{\mathcal{G}}(t)=\left(\begin{array}{cc}
0 & 0 \\
0 & \Delta_{k}(t)
\end{array}\right)+\Phi_{k}(t)
$$

where $\Phi_{k}(t): \mathcal{G}_{k} \rightarrow \mathcal{G}_{k}$ is given by

$$
\Phi_{k}(t):=\left(\begin{array}{l|l}
\Delta_{k-1}^{c o m b}+g_{k-1 ; s}(t) g_{k-1 ; s}(t)^{*} & -\delta_{k-1}^{*} g_{k ; s}(t)+g_{k-1 ; s}(t) d_{k-1}^{*}(t) \\
\hline-g_{k ; s}(t)^{*} \delta_{k-1}+d_{k-1}(t) g_{k-1 ; s}(t)^{*} & g_{k ; s}(t)^{*} g_{k ; s}(t)
\end{array}\right)
$$

As $g_{k ; s}(t)$ and $g_{k ; s}(t)^{*}$ are bounded operators of trace class for $s>\frac{n}{2}+1$ (cf Proposition 2.5) the maps $g_{k ; s}(t) d_{k}^{*}(t)$ and $d_{k}(t) g_{k ; s}(t)^{*}$ are bounded operators of trace class for $s>n / 2+1$ and we conclude that $\Phi_{k}(t)$ is a bounded operator of trace class. By Proposition 1.5 and its remark, we conclude that

$$
\begin{aligned}
& F \cdot p \cdot z=0\left(\frac{1}{\Gamma(z)} \int_{0}^{1} \lambda^{z-1} \operatorname{Tr}\left(\left(\begin{array}{cc}
0 & 0 \\
0 & h
\end{array}\right) e^{-\lambda \Delta_{k}^{\mathcal{G}}(t)}\right) d \lambda\right)= \\
& =F \cdot p \cdot z=0\left(\frac{1}{\Gamma(z)} \int_{0}^{1} \lambda^{z-1} \operatorname{Tr}\left(h e^{-\lambda \Delta_{k}(t)}\left(I d-P_{\Delta_{k}(t)}(\{0\})\right)\right) d \lambda\right.
\end{aligned}
$$

where $P_{\Delta_{k}(t)}(\{0\})$ denotes the orthogonal projection onto $\operatorname{Ker} \Delta_{k}(t)$.

One verifies (cf [BZ, p 81]) that $e^{t h} \Delta_{k}(t) e^{-t h}$ are equal to the Laplacians $\Delta_{k}^{\prime}$ associated to the undeformed deRham complex, the Riemannian metric $g$ and the Hermitian structure $e^{-2 t h} \mu$.

Combining this together with (2.30)-(2.32), we obtain 


$$
\begin{aligned}
& \frac{d}{d t} \log \mathcal{R}(t)= \\
& \mathbf{( 2 . 3 3 )}=\sum_{k}(-1)^{k} F \cdot p \cdot z=0 \\
& =\sum_{k}(-1)^{k} F \cdot p \cdot z=0 \\
\Gamma(z) & \left.\int_{0}^{1} \lambda^{z-1} \operatorname{Tr}\left(h e^{-\lambda \Delta_{k}(t)}\left(I d-P_{\Delta_{k}(t)}(\{0\})\right)\right) d \lambda\right) \\
\Gamma(z) & \left.\int_{0}^{1} \lambda^{z-1} \operatorname{Tr}\left(h e^{-\lambda \Delta_{k}^{\prime}}\left(I d-P_{\Delta_{k}^{\prime}}(\{0\})\right)\right) d \lambda\right)
\end{aligned}
$$

where we used that, as $\Delta_{k}(t)=e^{-t h} \Delta_{k}^{\prime} e^{t h}, e^{-\lambda \Delta_{k}(t)}=e^{-t h} e^{-\lambda \Delta_{k}^{\prime}} e^{t h}$ and $e^{t h}\left(I d-P_{\Delta_{k}(t)}(\{0\})\right) e^{-t h}=I d-P_{\Delta_{k}^{\prime}}(\{0\})$.

It is known (cf e.g. [BFKM]) that the restriction $K_{k}(x ; \lambda, t)$ of the Schwartz kernel of $e^{-\lambda \Delta_{k}^{\prime}}\left(I d-P_{\Delta_{k}^{\prime}}(\{0\})\right)$ to the diagonal has an asymptotic expansion with respect to $\lambda$ for $\lambda \searrow 0$ of the form $\sum_{j>0} A_{k ; n-j}(t) \lambda^{-\frac{n-j}{2}}$ where $A_{k ; n-j}(t)$ are smooth densities on $M$ with values in $\operatorname{End}\left(\Lambda^{k}\left(T^{*} M\right) \otimes \mathcal{E}\right)$, depending on the parameter $\boldsymbol{t}$. Substituting into (2.33), and integrating with respect to $\lambda$ leads to

$$
\begin{aligned}
& \frac{d}{d t} \log \mathcal{R}(t)=\sum_{k}(-1)^{k} \operatorname{Tr}\left(h A_{k ; 0}(t)\right) \\
& =\operatorname{Tr}\left(h\left(\sum_{k}(-1)^{k} A_{k ; 0}(t)\right)\right) \\
& =\operatorname{dim} \mathcal{E}_{x} \operatorname{Tr}(h e(M, g))
\end{aligned}
$$

where $e(M, g)$ is the Euler form, which can be proven to be equal to $\frac{1}{\operatorname{dim} \mathcal{E}_{x}} \sum_{k}$ $(-1)^{k} A_{k ; 0}(t)$. If $\operatorname{dim} M$ is odd, $e(M, g) \equiv 0$.

\section{Proof of Proposition 0.1 in the case $g=g^{\prime}$}

\subsection{Outline of the Proof}

In this section we prove Proposition 0.1 in the case where the Riemannian metric $g$ is the same as the metric $g^{\prime}$ of the generalized triangulation $\tau=$ $\left(h, g^{\prime}\right)$. Throughtout this section we assume that the Hermitian structure $\mu$ is $\tau$-admissible, i.e. that there exists a neighborhood $U_{h}$ of the critical points of $h$ so that on $U_{h}, \mu$ is parallel with respect to the canonical flat connection on $\mathcal{E} \rightarrow M$.

From Theorem 2.9 we know that

(A) $\log \mathcal{R}(t)=\log \mathcal{R}+\int_{0}^{t} \frac{d}{d t} \log \mathcal{R}(t) d t$ is an affine function of $t$,

hence $\log \mathcal{R}(t)$ has an asymptotic expansion with $\log \mathcal{R}$ as the free term.

Recall from the proof of Proposition 2.1 (iii) that, for $t$ sufficiently large, $\left(H_{0}(\Lambda(M ; \mathcal{E})), d(t)\right)$ is the direct sum of two subcomplexes,

$$
\left(H_{0}(\Lambda(M ; \mathcal{E})), d(t)\right)=\left(\Omega_{s m}(t), d(t)\right) \oplus\left(H_{0, l a}(t), d(t)\right)
$$

where

$$
H_{k ; 0}:=H_{0}\left(\Lambda^{k}(M ; \mathcal{E})\right),
$$




$$
\Omega_{s m}^{k}(t)=Q_{k}(t)\left(H_{k ; 0}\right),
$$

$H_{k ; 0, l a}(t)=\left(I d-Q_{k}(t)\right)\left(H_{k ; 0}\right)$ and $Q_{k}(t)$ denotes the orthogonal spectral projector $Q_{k}(t): H_{k ; 0} \rightarrow H_{k ; 0}$ of the k-Laplacian $\Delta_{k}(t)$ corresponding to the interval $[0,1]$. Notice that $\Omega_{s m}^{k}(t)$ consists of smooth forms and that $\left(H_{0, l a}(t), d(t)\right)$ is an algebraically acyclic $\zeta$-regular complex of sF type. Notice also that

$\left(\Omega_{s m}(t), d(t)\right)$ is a $\zeta$-regular complex of finite type for $t$ large enough. The finite type property follows from [BFKM] Theorem 5.5. Denote by $g_{s, s m}(t)$ the restriction of $g_{s}(t)$ to $\Omega_{s m}(t)$. This is a morphism of trace class for $s>\frac{n}{2}+1$ since $g_{s}(t)$ is of trace class by Proposition 2.5. We have the following short exact sequence of algebraically acyclic, $\zeta$-regular complexes of $\mathrm{sF}$ type,

$$
0 \rightarrow \mathcal{C}\left(g_{s, s m}(t)\right) \stackrel{I}{\rightarrow} \mathcal{C}\left(g_{s}(t)\right) \stackrel{P}{\rightarrow} H_{0, l a}(t) \rightarrow 0
$$

where $I$, resp. $P$, is the obvious inclusion, resp. projection. One verifies in a straightforward way that (3.1) satisfies the assumptions in Lemma 1.14 and therefore, with

$$
\mathcal{R}_{s m}(t):=T\left(\mathcal{C}\left(g_{s, s m}(t)\right)\right),
$$

we obtain the decomposition

(B) $\log \mathcal{R}(t)=\log \mathcal{R}_{s m}(t)+\log T\left(H_{0, l a}(t)\right)$.

For consistency with the notation in [BFK2] and [BFKM] we write

$$
\log T_{l a}(t):=\log T\left(H_{0, l a}(t)\right) .
$$

The two terms, $\log \mathcal{R}_{s m}(t)$ and $\log T_{l a}(t)$, will each be treated separately. To obtain an asymptotic expansion of $\log \mathcal{R}_{s m}(t)$ as $t \rightarrow \infty$, we proceed in two steps. Recall that $f(t)$ is given by the composition

$$
f(t):\left(\Omega_{s m}(t), d(t)\right) \stackrel{e^{t h}}{\rightarrow}(\Omega, d) \stackrel{\operatorname{Int}}{\rightarrow}(\mathcal{C}, \delta) .
$$

We show in subsection 3.2, Proposition 3.1 (i), that

$$
\mathcal{R}_{s m}(t)=T(\mathcal{C}(f(t))) .
$$

The morphism $f(t)$, unlike $g_{s, s m}(t)$, can be studied using the Witten-HelfferSjöstrand theory (cf [BFK2, section 3], also [BFKM, section 5]). We show in subsection 3.2, Proposition 3.1 (ii) that $\log T(\mathcal{C}(f(t)))$ has an asymptotic expansion which we calculate up to terms of order $0\left(\frac{1}{t}\right)$. Proposition 3.1 implies that:

(C) $\log \mathcal{R}_{\text {sm }}(t)$ admits an asymptotic expansion for $t \rightarrow \infty$ of the form 


$$
\begin{aligned}
\log \mathcal{R}_{s m}(t) & =\operatorname{dim} \mathcal{E}_{x} \cdot\left(\sum_{j}(-1)^{j} \sum_{x \in C r_{j}(h)} h(x)\right) t \\
& -\left(\sum_{j}(-1)^{j}\left(\frac{n}{4}-\frac{j}{2}\right) m_{j} \cdot \operatorname{dim} \mathcal{E}_{x}\right) \log \left(\frac{\pi}{t}\right)+0\left(\frac{1}{t}\right)
\end{aligned}
$$

Concerning $\log T_{l a}(t)$, we will establish in subsection 3.3:

(D) (i) $\log T_{l a}(t)$ admits an asymptotic expansion as $t \rightarrow \infty$ and

$$
F T_{t=\infty}\left(\log T_{l a}(t)\right)=-F T_{t=\infty}\left(\log \mathcal{R}_{s m}(t)\right)+\int_{M} \alpha
$$

where $\alpha$ is a local density on $M$ which vanishes in a neighborhood of the critical points of $h$.

(ii) If $\mu$ is parallel with respect to the canonical flat connection, $\int_{M} \alpha=0$.

The results (A)-(D) prove Proposition 0.1 in the case where $g=g^{\prime}$.

\subsection{Asymptotic expansion of $\log \mathcal{R}_{s m}(t)$}

In this subsection we prove the statement (C) of subsection 3.1. By the WittenHelffer-Sjöstrand theory, $f(t):=I n t \cdot e^{t h}$ is an isomorphism of cochain complexes for $t$ sufficiently large. Therefore the mapping cone $\mathcal{C}(f(t))$ is an algebraically acyclic, $\zeta$-regular complex of $\mathrm{sF}$ type and thus admits a torsion, $T(\mathcal{C}(f(t)))$.

Proposition 3.1 For $t$ sufficiently large,

(i) $\log \mathcal{R}_{s m}(t)=\log T(\mathcal{C}(f(t)))$;

(ii) $\log T(\mathcal{C}(f(t)))$ admits an asymptotic expansion for $t \rightarrow \infty$ of the form

$$
\begin{aligned}
\log T(\mathcal{C}(f(t))) & =\operatorname{dim} \mathcal{E}_{x} \cdot\left(\sum_{j}(-1)^{j} \sum_{x \in C r_{j}(h)} h(x)\right) t \\
& -\left(\sum_{j}(-1)^{j}\left(\frac{n}{4}-\frac{j}{2}\right) m_{j} \operatorname{dim} \mathcal{E}_{x}\right) \log \left(\frac{\pi}{t}\right)+0\left(\frac{1}{t}\right) .
\end{aligned}
$$

Proof (i) Notice that the morphism $f(t)$ is equal to the composition $(s>n+2)$

$$
\left(\Omega_{s m}(t), d(t)\right) \stackrel{e_{s}^{t h}}{\rightarrow} H_{s}(\Lambda(M ; \mathcal{E})) \stackrel{\text { Inţs }}{\longrightarrow} \mathcal{C}(M, \tau, \mathcal{T})
$$

whereas $g_{s, s m}(t)$ is given by

$$
\left(\Omega_{s m}(t), d(t)\right) \stackrel{e^{t h}}{\rightarrow}\left(H_{0}(\Lambda(M ; \mathcal{E})), d\right) \stackrel{(\Delta+I d)^{-s / 2}}{\rightarrow}\left(H_{s}(\Lambda(M ; \mathcal{E})), d\right)
$$




$$
\stackrel{\text { Ints }}{\rightarrow} \mathcal{C}(M, \tau, \mathcal{T}) \text {. }
$$

By Proposition 2.5, Int $t_{s}$ is a morphism of trace class, multiplication by $e^{t h}$ is a morphism of trace class as $\Omega_{s m}(t)$ is a cochain complex of finite rank (cf Examples 1.6) and $(\Delta+I d)^{-s / 2}$ is an isometry. Each of them induces an isomorphism in algebraic cohomology (cf Proposition 2.1). Therefore we can apply Proposition 1.15 to (3.3) and (3.4) to obtain

$$
\log T(\mathcal{C}(f(t)))=\log T\left(\mathcal{C}\left(e_{s}^{t h}\right)\right)+\log T\left(\mathcal{C}\left(\text { Int }_{s}\right)\right)
$$

and

(3.6) $\log T\left(\mathcal{C}\left(g_{s, s m}(t)\right)\right)=\log T\left(\mathcal{C}\left(e^{t h}\right)\right)+\log T\left(\mathcal{C}\left(\operatorname{Int}_{s}(\Delta+I d)^{-s / 2}\right)\right)$.

In view of Proposition 1.16 and the fact that $(\Delta+I d)^{-s / 2}$ is an isometry we conclude that

$$
\log T\left(\mathcal{C}\left(\operatorname{Int}_{s}\right)\right)=\log T\left(\mathcal{C}\left(\operatorname{Int}_{s}(\Delta+I d)^{-s / 2}\right)\right) .
$$

Further, $e^{t h}=I n_{s, 0} e_{s}^{t h}$ is a morphism

$$
\left(\Omega_{s m}(t), d(t)\right) \stackrel{e_{s}^{t h}}{\longrightarrow} H_{s}(\Lambda(M ; \mathcal{E})) \stackrel{I n_{s}, 0}{\longrightarrow} H_{0}(\Lambda(M ; \mathcal{E}))
$$

with $I n_{s, 0}$ and $e_{s}^{t h}$ being both morphisms of trace class and inducing isomorphisms in algebraic cohomology (cf Proposition 2.1). Thus, applying Proposition 1.15 once more, we obtain, in view of Proposition 2.3,

$$
\log T\left(\mathcal{C}\left(e^{t h}\right)\right)=\log T\left(\mathcal{C}\left(e_{s}^{t h}\right)\right) .
$$

Combining (3.5) - (3.8) leads to the proof of statement (i).

(ii) Recall from [BFK2, section 3.3] the following commutative diagram (for $t$ sufficiently large)

$$
\left(\Omega_{s m}(t), d(t)\right) \stackrel{f(t)}{\longrightarrow}(\mathcal{C}, \delta)
$$

$$
\Phi+0\left(\frac{1}{t}\right) \searrow \quad \downarrow S(t)
$$

where $\Phi$ is a morphism which is an isometry, $\tilde{\delta}=\tilde{\delta}(t)$ is chosen so that the diagram above is commutative, $\tilde{\delta}_{k}(t):=S_{k+1}(t)^{-1} \cdot \delta_{k} \cdot S_{k}(t)$, and $S(t)$ is the morphism given by

$$
S_{k}(t)=\left(\bigoplus_{x \in C r_{k}(h)} S_{x}(t)\right): \mathcal{C}^{k} \longrightarrow \mathcal{C}^{k}
$$


where $\mathcal{C}^{k}:=\bigoplus_{x \in C r_{k}(h)} \mathcal{E}_{x}$ and $S_{x}(t): \mathcal{E}_{x} \rightarrow \mathcal{E}_{x}$ is given by $S_{x}(t):=e^{-t h(x)}\left(\frac{\pi}{t}\right)^{\frac{n}{4}-\frac{k}{2}} I d$.

For $t$ sufficiently large, $S(t), f(t), \Phi+0\left(\frac{1}{t}\right)$ are isomorphisms of cochain complexes. Thus, by Lemma 1.11 , the mapping cones $\mathcal{C}\left(\Phi+0\left(\frac{1}{t}\right)\right), \mathcal{C}(S(t))$ and $\mathcal{C}(f(t))$ are algebraically acyclic. By Proposition 1.15,

$$
\log T\left(\mathcal{C}\left(\Phi+0\left(\frac{1}{t}\right)\right)\right)=\log T(\mathcal{C}(f(t)))+\log T(\mathcal{C}(S(t))) .
$$

Using Proposition 1.12, one verifies that $\log T\left(\mathcal{C}\left(I d+0\left(\frac{1}{t}\right)\right)\right)=0\left(\frac{1}{t}\right)$.

Therefore

$$
\log T(\mathcal{C}(f(t)))=-\log T(\mathcal{C}(S(t)))+0\left(\frac{1}{t}\right) .
$$

By Proposition 1.12,

$$
\log T(\mathcal{C}(S(t)))=\sum(-1)^{j} \sum_{x \in C r_{j}(h)} \log \left(e^{-t h(x)}\left(\frac{\pi}{t}\right)^{\frac{n}{4}-\frac{j}{2}}\right) \operatorname{dim} \mathcal{E}_{x} .
$$

In view of (3.10) we obtain

$$
\begin{aligned}
\log \mathcal{C}(f(t))= & \left(\sum(-1)^{j} \sum_{x \in C r_{j}(h)} h(x) \operatorname{dim} \mathcal{E}_{x}\right) t \\
& -\left(\sum(-1)^{j}\left(\frac{n}{4}-\frac{j}{2}\right) m_{j} \operatorname{dim} \mathcal{E}_{x}\right) \log \frac{\pi}{t}+0\left(\frac{1}{t}\right) .
\end{aligned}
$$

\subsection{Asymptotic expansion of $\log T_{l a}(t)$}

In this section we prove the statement (D) in subsection 3.1. As $\log T_{l a}(t)=$ $\log \mathcal{R}(t)-\log \mathcal{R}_{s m}(t)$ and in view of Theorem 2.9 and Proposition 3.1, $\log T_{l a}(t)$ admits an asymptotic expansion for $t \rightarrow \infty$.

The arguments to prove (D) follow closely the ones in [BFKM, section 6.2]: we will derive statement (D) from a relative version of this statement, Proposition 3.2, and from the validity of (D) in some simple cases.

We consider systems $\mathcal{F}:=(M, \mathcal{E}, \nabla, \mu, g, \tau)$, where $(\mathcal{E}, \nabla)$ is a bundle of $\mathcal{A}$ -Hilbert modules equipped with a flat connection, $\mu$ a Hermitian structure, $g$ a Riemannian metric and $\tau=\left(h, g^{\prime}\right)$ a generalized triangulation with the metric $g^{\prime}=g$. We suppose that $\mu$ is admissible with respect to $\tau$, i.e there exists an open neighborhood $U_{h}$ of the critical points so that on $U_{h}, \mu$ is parallel with respect to the connection $\nabla$. Introduce $V_{\mathcal{F}}(t, \epsilon):=\frac{1}{2} \sum_{q}(-1)^{q+1} q \log \operatorname{det}\left(\Delta_{q}(t)+\epsilon\right)$. 
Proposition 3.2 For any $\epsilon>0$ there exists a smooth density $\alpha_{\mathcal{F}}(\epsilon)$ on $M \backslash C r(h)$, which is polynomial in $\epsilon$ and a local quantity (cf subsection 0.2 and [BFKM] section 2) so that the following statement hold:

(i) If $\mathcal{F}^{\prime}:=\left(M, \mathcal{E}, \nabla, \mu, g, \tau_{\mathcal{D}}\right)$ denotes the system obtained from $\mathcal{F}$ by replacing the triangulation $\tau=(h, g)$ by its dual $\tau_{D}=(n-h, g)(n=\operatorname{dim} M)$, then

$$
\alpha_{\mathcal{F}}(\epsilon)+(-1)^{n+1} \alpha_{\mathcal{F}^{\prime}}(\epsilon)=0 .
$$

(ii) If $\mathcal{F}$ and $\tilde{\mathcal{F}}$ are two systems as above and $\mathcal{F} \otimes \tilde{\mathcal{F}}$ denotes the system defined by

$$
\mathcal{F} \otimes \tilde{\mathcal{F}}:=(M \times \tilde{M}, \mathcal{E} \otimes \tilde{\mathcal{E}}, \bar{\nabla}, \mu \otimes \tilde{\mu}, g \times \tilde{g}, \tau \times \tilde{\tau})
$$

with $\bar{\nabla}=\nabla \otimes i d+i d \otimes \tilde{\nabla}$, then

$$
\alpha_{\mathcal{F} \otimes \tilde{\mathcal{F}}}(\epsilon)=\left(\alpha_{\mathcal{F}}(\epsilon) \otimes e(\tilde{M}, \tilde{g})+e(M, g) \otimes \tilde{\alpha}_{\tilde{\mathcal{F}}}(\epsilon)\right) \operatorname{dim} \mathcal{W},
$$

where $e(M, g)$ denotes the Euler form of $(M, g)$.

(iii) The density $\alpha_{\mathcal{F}}(\epsilon)$ vanishes on $U_{h} \backslash C r(h)$.

(iv) Assume $\mathcal{F}$ and $\tilde{\mathcal{F}}$ are two systems as above so that $\sharp \mathcal{C} r_{q}(h)=\sharp C r_{q}(\tilde{h})(0 \leq$ $q \leq n)$, and the bundles $\mathcal{E}$ and $\tilde{\mathcal{E}}$ have isomorphic $\mathcal{A}$-Hilbert modules as fibers. Then $V_{\mathcal{F}}(t, \epsilon)-V_{\tilde{\mathcal{F}}}(t, \epsilon)$ has an asymptotic expansion with

(a) $F T_{t=\infty}\left(V_{\mathcal{F}}(t, \epsilon)-V_{\tilde{\mathcal{F}}}(t, \epsilon)\right)=\int_{M \backslash C r(h)} \alpha_{\mathcal{F}}(\epsilon)-\int_{\tilde{M} \backslash C r(\tilde{h})} \alpha_{\tilde{\mathcal{F}}}(\epsilon)$;

(b) $F T_{t=\infty}\left(\log T_{l a}(t)\right)-F T_{t=\infty}\left(\log \tilde{T}_{l a}(t)\right)=\int_{M \backslash C r(h)} \alpha_{\mathcal{F}}(0)-\int_{\tilde{M} \backslash C r(\tilde{h})} \alpha_{\tilde{\mathcal{F}}}(0)$.

Proof: We begin with the construction of the density $\alpha_{\mathcal{F}}(\epsilon)$. Away from the critical points of $h$ we choose a coordinate system for $\mathcal{E} \rightarrow M$. In these coordinates we calculate inductively the quantities $r_{-2-j}^{q}(h, \epsilon ; x, \xi, t, \mu)$, by the formulae 6.59 in [BFKM], from the symbol of the operator with parameter $\Delta_{q}(t)+\epsilon$, which is elliptic with parameter $t$ away from $C r(h)$ (cf [BFKM] (3.2)). We are interested in the quantity $r_{-2-n}^{q}$ only. We use formula (3.4) in [BFKM] to obtain from $r_{-2-n}^{q}$ the density $\alpha_{\mathcal{F}}^{q}(h, \epsilon)$ on $M \backslash C r(h)$. We define

$$
\alpha_{\mathcal{F}}(h, \epsilon):=1 / 2 \sum_{q}(-1)^{q+1} q \alpha_{\mathcal{F}}^{q}(h, \epsilon) .
$$

Since by construction $r_{-2-n}^{q}$ is a polynomial in $\epsilon$ of degree smaller than $n$ so is $\alpha_{\mathcal{F}}(h, \epsilon)$.

(i) follows from the following homogeneity property (cf [BFKM] (6.60)):

$$
r_{-2-n}(h, x,-\xi,-t, \mu)=(-1)^{n} r_{-2-n}(h, x, \xi, t, \mu)
$$

and by a straightforward verification

$$
r_{-2-n}(n-h, x, \xi, t, \mu)=r_{-2-n}(h, x, \xi,-t, \mu) .
$$


To prove (ii) we consider systems $\mathcal{G}=(M, \mathcal{E}, \nabla, \mu, g, \omega)$, where $M, \mathcal{E}, \nabla, \mu, g$ are as above and $\omega$ is a closed 1-form on $M$. A system $\mathcal{F}=(M, \mathcal{E}, \nabla, \mu, g, \tau=$ $(g, h))$ gives rise to a system $\mathcal{G}$ by taking $\omega=d h$. For any such $\mathcal{G}$, define the Witten deformation by taking $\omega$ instead of $d h$ and then the Witten Laplacians $\Delta_{q}(t)$ for any real number $t$. If the 1-form $\omega$ has no zeros, $\Delta_{q}(t)$ is elliptic with parameter and the general theory in [BFKM], section 2 implies that for any fixed $\epsilon>0, V_{\mathcal{G}}(t, \epsilon):=\frac{1}{2} \sum_{q}(-1)^{q+1} q \log \left(\Delta_{q}(t)+\epsilon\right)$ has an asymptotic expansion for $t \rightarrow \infty$, whose free term is $\int_{M} \alpha_{\mathcal{G}}$, with $\alpha_{\mathcal{G}}$ a local density on $M$. Following [BMFK] section 2 this density is calculated in the same way as the density $\alpha_{\mathcal{F}}$ (using $\omega$ instead of $d h$ ).

Since $\left.\mathcal{F}\right|_{M \backslash C r(h)}$ is locally isomorphic to the restriction to an open set of a system $\mathcal{G}=(\tilde{M}, \tilde{\mathcal{E}}, \tilde{\nabla}, \tilde{\mu}, \tilde{g}, \tilde{\omega})$ such that $\tilde{M}$ is closed and $\tilde{\omega}$ has no zeros, it suffices to check (3.11) for $\alpha_{\mathcal{G}}$ and $\alpha_{\tilde{\mathcal{G}}}$ instead of $\alpha_{\mathcal{F}}$ and $\alpha_{\tilde{\mathcal{F}}}$.

For a system $\mathcal{G}$ and $u>0, t>0$ consider the operator $e^{-u \Delta_{q}(t)}$ which is a smoothing operator and denote by $\lambda_{q}(u, t)$ the pointwise (von Neumann)trace of the restriction of its Schwartz kernel to the diagonal. This provides a two parameter family (in $u$ and $t$ ) of densities on $M$. Denote by

$$
\lambda_{\mathcal{G}}(u, t):=1 / 2 \sum_{q}(-1)^{q+1} q \lambda_{q}(u, t) .
$$

Define the smooth three parameter family, $\eta(s, t, \epsilon)$, of densities on $M$ for $\Re e s$ sufficiently large,

$$
\eta(s, t, \epsilon)=\frac{1}{\Gamma(s)} \int_{0}^{\infty} u^{s-1} e^{-u \epsilon} \lambda_{\mathcal{G}}(u, t) d u .
$$

which, by analytic continuation, is holomorphic in $s$ near $0 \in \mathbb{C}$. Denote by $\theta_{\mathcal{G}}(t, \epsilon)$ the density valued function in $t$ and $\epsilon$,

$$
\theta_{\mathcal{G}}(t, \epsilon):=\left.\frac{d}{d s}\right|_{s=0} \eta(s, t, \epsilon) .
$$

For the system $\mathcal{G} \otimes \tilde{\mathcal{G}}$, we can prove that

$$
\theta_{\mathcal{G} \otimes \tilde{\mathcal{G}}}(t, \epsilon)=\theta_{\mathcal{G}}(t, \epsilon) \otimes e(\tilde{M}, \tilde{g}) \operatorname{dim} \mathcal{W}+e(M, g) \otimes \theta_{\tilde{\mathcal{G}}}(t, \epsilon) \operatorname{dim} \mathcal{W}
$$

This can be derived as in the proof of Proposition 2.4 in [BFKM] from the following facts:

(i) $\Delta_{q}^{\mathcal{G} \otimes \tilde{\mathcal{G}}}(t)=\Delta_{q}^{\mathcal{G}} \otimes I d+I d \otimes \Delta_{q}^{\tilde{\mathcal{G}}}(t)$,

whose proof is similar to the proof of Proposition 2.4 in [BFKM] and for any $t$, (ii) $\eta(0, t, \epsilon=0)=e(M, g) \operatorname{dim} \mathcal{W}$,

whose proof follows from the local index theorem of Bismut and Zhang. This takes care of (3.12).

For (iii), we use the locality of the density $\alpha_{\mathcal{F}}(\epsilon)$ and the explicit formula of $\Delta_{q}(t)$. In admissible coordinates in a neighborhood of the critical points $\Delta_{q}(t)$, 
is the same as $\Delta_{q}(t)$, for a product of systems $\mathcal{G}$ with underlying manifolds of dimension one. Using (3.12) and the vanishing of the Euler form on $M=\mathbb{R}$, the result follows.

(iv) (a) is actually Proposition 6.6 in [BFKM] and (b) follows from Lemma 6.7 of [BFKM].

Proof of statement (D). We want to apply Proposition 3.2. Set $\tilde{M}:=M, \tilde{\tau}:=$ $\tau, g=\tilde{g}, \tilde{\rho}$ the trivial representation of $\Gamma \equiv \pi_{1}(M)$ on $\mathcal{W}$. Then the associated bundle $\tilde{\mathcal{E}} \rightarrow M$ is trivial ; we choose $\tilde{\mu}$ the trivial Hermitian structure. Then by (2.15) and the equality of analytic and Reidemeister torsion for the trivial representation we have $\tilde{\mathcal{R}}=1$. Since $\log \tilde{\mathcal{R}}(t)$ admits an asymptotic expansion by $(B)$ so does $\log \tilde{T}_{l a}(t)$. Since the free term of the expansion of $\log \tilde{\mathcal{R}}$ is zero, one obtains

$$
F T_{t=\infty} \log \tilde{T}_{l a}(t)=\left(\sum_{j}(-1)^{j}\left(\frac{n}{4}-\frac{j}{2}\right) m_{j} \operatorname{dim} \mathcal{E}_{x}\right) \log \pi .
$$

Statement (i) in (D) follows then from Proposition 3.2 (iv) . To prove statement (ii), observe that since $\mu$ is parallel with respect to the canonical flat connection $\nabla$, the Hodge $*$ operator induces an isometry between $L_{2}\left(\Lambda^{k}(M ; \mathcal{E})\right)$ and $L_{2}\left(\Lambda^{n-k}(M ; \mathcal{E})\right)$ and conjugates $\Delta_{k}(t)$ with $\Delta_{n-k}(-t)$. Thus $\Delta_{k}(t)$ and $\Delta_{n-k}(t)$ are isospectral and then by the definition of $V_{\mathcal{F}}(t, \epsilon)$ we have :

$$
V_{\mathcal{F}}(t, \epsilon)=(-1)^{n+1} V_{\mathcal{F}^{\prime}}(t, \epsilon)
$$

and

$$
V_{\tilde{\mathcal{F}}}(t, \epsilon)=(-1)^{n+1} V_{\tilde{\mathcal{F}}^{\prime}}(t, \epsilon) .
$$

Therefore

$$
V_{\mathcal{F}}(t, \epsilon)-V_{\tilde{\mathcal{F}}^{\prime}}(t, \epsilon)=(-1)^{n+1} V_{\mathcal{F}^{\prime}}(t, \epsilon)-(-1)^{n+1} V_{\tilde{\mathcal{F}}^{\prime}}(t, \epsilon),
$$

and thus by Proposition 3.2 (iv) we have

$$
\begin{gathered}
F T_{t=\infty} \log T_{l a}(\mathcal{F}, t)-F T_{t=\infty} \log T_{l a}(\tilde{\mathcal{F}}, t)= \\
=(-1)^{n+1} F T_{t=\infty} \log T_{l a}\left(\mathcal{F}^{\prime}, t\right)-(-1)^{n+1} F T_{t=\infty} \log T_{l a}\left(\tilde{\mathcal{F}}^{\prime}, t\right) .
\end{gathered}
$$

Again by Proposition 3.2 (iv)

$$
\begin{gathered}
\int_{M \backslash C r(h)} \alpha_{\mathcal{F}}(0)-\int_{M \backslash C r(h)} \alpha_{\tilde{\mathcal{F}}^{(}}(0)= \\
(-1)^{n+1}\left(\int_{M \backslash C r(h)} \alpha_{\mathcal{F}^{\prime}}(0)-\int_{M \backslash C r(h)} \alpha_{\tilde{\mathcal{F}}^{\prime}}(0)\right) .
\end{gathered}
$$


In view of (3.12), (D) (i) and

$$
\int_{M \backslash C r(h)} \alpha_{\tilde{\mathcal{F}}}(0)=\int_{M \backslash C r(h)} \alpha_{\tilde{\mathcal{F}}^{\prime}}(0)=0,
$$

because $\mu$ is parallel, one obtains

$$
\int_{M \backslash C r(h)} \alpha_{\mathcal{F}}(0)=(-1)^{n+1} \int_{M \backslash C r(h)} \alpha_{\mathcal{F}^{\prime}}(0) .
$$

Statement (D)(ii) follows by combining this last formula with Proposition 3.2 (iv)

\section{Anomalies of the relative torsion}

\subsection{Metric and Hermitian anomalies}

In this subsection, we investigate how the relative torsion $\mathcal{R}=\mathcal{R}(M, \rho, \mu, g, \tau)$ varies with respect to the Riemannian metric $g$ and the Hermitian structure $\mu$. We denote by $\mathcal{E} \rightarrow M, \nabla$ the flat bundle associated to $\rho$. In order to formulate the results we introduce a number of additional quantities:

The closed 1-form $\theta=\theta(\rho, \mu) \in \Omega^{1}(M)$ : Choose a finite covering $M$ by open sets $\left(U_{j}\right)_{j \in J}$, which are simply connected, and points $x_{j} \in U_{j}$. Define $v_{j}: U_{j} \rightarrow \mathbb{R}$ by

$$
v_{j}(x):=\log \operatorname{vol}\left(T_{x, x_{j}}\right)=\frac{1}{2} \log \operatorname{det}\left(T_{x, x_{j}}^{*} T_{x, x_{j}}\right)
$$

where $T_{x, x_{j}}:\left(\mathcal{E}_{x}, \mu_{x}\right) \rightarrow\left(\mathcal{E}_{x_{j}}, \mu_{x_{j}}\right)$ denotes the parallel transport from $\mathcal{E}_{x}$ to $\mathcal{E}_{x_{j}}$ along any curve joining $x$ and $x_{j}$ inside $U_{j}$. (As the connection $\nabla$ of $\mathcal{E} \rightarrow M$ is flat, the map $T_{x, x_{j}}$ does not depend on the choice of the curve.) Denote by $\theta=\theta(\rho, \mu)$ the smooth 1 -form on $M$ defined by $\theta_{j}:=d v_{j}$. Notice that if the Hermitian structure $\mu$ is parallel with respect to the canonical flat connection $\nabla$, then $\theta(\mu)=0$. We mention that in [BZ] the quantity $\theta$ is defined to be $2 \theta(\rho, \mu)$.

If the representation $\rho$ is unimodular, (i.e. $\log \operatorname{vol}(\rho(g))=0$ for any $g \in \Gamma$ ) then one can find $\mu$ so that $\theta(\rho, \mu)=0$. To see this let $\operatorname{det}_{\mathbb{R}} \mathcal{E} \rightarrow M$ denote the 1-dimensional real vector bundle obtained by assigning to each point $x \in M$ the vector space $\operatorname{det}_{\mathbb{R}} \mathcal{E}_{x}$, as described in [CFM], and let $\operatorname{det} \nabla$ denote the induced flat connection in $\operatorname{det}_{\mathbb{R}} \mathcal{E} \rightarrow M$. This is the flat bundle associated with the representation $\operatorname{det}_{\mathbb{R}} \rho$. The unimodularity of $\rho$ implies the existence of a parallel section $s_{0}$ in $\operatorname{det}_{\mathbb{R}} \mathcal{E} \rightarrow M$. Take a Hermitian structure $\mu$ in $\mathcal{E} \rightarrow M$, and denote by $s$ the tautological section in $\operatorname{det}_{\mathbb{R}} \mathcal{E} \rightarrow M$ induced by $\mu$. Clearly there exists a smooth nonzero function $f: M \rightarrow \mathbb{R}_{+}$so that $s_{0}=f \cdot s$. If $\mu$ is 
parallel above the open set $U$, then $\left.f\right|_{U}=1$. It is immediate from definition of $\theta$ that $\theta(\rho, f \cdot \mu)=0$.

If $\left(\rho_{i}, \mu_{i}\right), i=1,2$ are two pairs consisting of a representation and a Hermitian structure on the induced flat bundle, then one can verify in a straightforward manner that

$$
\theta\left(\rho_{1} \otimes \rho_{2}, \mu_{1} \otimes \mu_{2}\right)=\theta\left(\rho_{1}, \mu_{1}\right) \otimes 1+1 \otimes \theta\left(\rho_{2}, \mu_{2}\right) .
$$

The function $V=V\left(\rho, \mu_{1}, \mu_{2}\right) \in \Omega^{0}(M)$ : If $\mu_{j}, j=1,2$ are two Hermitian structures of $\mathcal{E} \rightarrow M$, define the smooth function

$$
V(x):=V\left(\rho, \mu_{1}, \mu_{2}\right)(x)=\log \operatorname{vol}\left(\operatorname{Id}_{x}:\left(\mathcal{E}_{x}, \mu_{1}(x)\right) \rightarrow\left(\mathcal{E}_{x}, \mu_{2}(x)\right)\right) .
$$

Note that

$$
\theta\left(\rho, \mu_{1}\right)-\theta\left(\rho, \mu_{2}\right)=d V\left(\rho, \mu_{1}, \mu_{2}\right)
$$

$$
V\left(\rho, \mu_{1}, \mu_{2}\right)=-V\left(\rho, \mu_{2}, \mu_{1}\right)
$$

$$
V\left(\rho, \mu_{1}, \mu_{3}\right)=V\left(\rho, \mu_{1}, \mu_{2}\right)+V\left(\rho, \mu_{2}, \mu_{3}\right) .
$$

The Euler form $e(M, g) \in \Omega^{n}\left(M ; \mathcal{O}_{M}\right)$ : Denote by $e(M, g)$ the Euler form associated to the Levi-Cività connection on the tangent bundle $T M$. This is a form with coefficients in the orientation bundle of $M$, a flat, 1-dimensional real vector bundle.

The Chern-Simon element $\left[e_{C S}\left(M, g_{1}, g_{2}\right)\right] \in \Omega^{n-1}\left(M ; \mathcal{O}_{M}\right) / d \Omega^{n-2}\left(M ; \mathcal{O}_{M}\right)$ : For two Riemannian metrics $g_{1}$ and $g_{2}$ on $M$ denote by $\left[e_{C S}=e_{C S}\left(M, g_{1}, g_{2}\right)\right.$ ] the Chern-Simon class , cf [BZ], p 46. Recall that

$$
d\left(e_{C S}\left(M, g_{1}, g_{2}\right)\right)=e\left(M, g_{2}\right)-e\left(M, g_{1}\right)
$$

and that there is a canonical construction, due to Chern-Simon, for a representative $e_{C S}$ of $\left[e_{C S}\right]$ so that for a smooth 1-parameter family $g_{2}(u)$ of Riemannian metrics on $M, e_{C S}\left(g_{1}, g_{2}(u)\right)$ is a smooth 1-parameter family of $(n-1)$ forms.

The following object appears in Theorem 0.1 and is related to the previous quantities.

The form $\Psi(T M, g) \in \Omega^{n-1}\left(T M \backslash M ; \mathcal{O}_{T M}\right)$ : Let $\pi: T M \rightarrow M$ be the tangent bundle of $M$. Clearly, $\mathcal{O}_{T M}=\pi^{*} \mathcal{O}_{M}$. Following Mathai-Quillen [MQ] Theorem 6.4, or Bismut Zhang [BZ] Theorem 3.4, one can construct a smooth form $\Psi(T M, g) \in \Omega^{n-1}\left(T M \backslash M ; \mathcal{O}_{T M}\right)$ with the following properties:

$$
d \Psi(T M, g)=\pi^{*}(e(M, g))
$$

If $g_{1}$ and $g_{2}$ are two Riemannian metrics on $M$ then 


$$
\Psi\left(T M, g_{2}\right)-\Psi\left(T M, g_{1}\right)=\pi^{*} e_{C S}\left(M, g_{1}, g_{2}\right) \text { (modulo exact forms) }
$$

If $\lambda: T M \backslash M \rightarrow T M \backslash M$ denotes multiplication by the real number $\lambda \neq 0$ then

(4.2"') $\quad \lambda^{*}(\Psi(T M, g))=(\lambda /|\lambda|)^{n} \Psi(T M, g)$.

(4.2 $\left.{ }^{\text {iv }}\right)$ For $x \in M$ fixed, denote by $\omega$ the volume form on $T_{x} M$ and by $Y$ the Euler vectorfield on $T_{x} M$ ( in polar coordinates, $Y=r \frac{\partial}{\partial r}, r$ the distance to the origin). Then

$$
\left.\Psi(T M, g)\right|_{T_{x} M}=-\frac{\Gamma(n / 2)}{2 \pi^{n / 2}} \frac{\iota Y \omega}{|Y|^{n}} .
$$

It is easy to check (cf [BZ] ) that for two Riemannian manifolds $\left(M_{i}, g_{i}\right), i=$ 1,2 we have

$$
\begin{aligned}
& \Psi\left(T\left(M_{1} \times M_{2}\right), g_{1} \times g_{2}\right)=\Psi\left(T\left(M_{1}\right), g_{1}\right) \otimes e\left(M_{2}, g_{2}\right)+ \\
& +e\left(M_{1}, g_{1}\right) \otimes \Psi\left(T\left(M_{2}\right), g_{2}\right) .
\end{aligned}
$$

Proposition 4.1 (Metric anomaly of the relative torsion)

(i) $\log \mathcal{R}\left(g_{2}\right)-\log \mathcal{R}\left(g_{1}\right)=-\int_{M} \theta \wedge e_{C S}\left(g_{1}, g_{2}\right)$.

(ii) If $\operatorname{dim} M$ is odd or $\mu$ is a Hermitian structure parallel with respect to the canonical flat connection, the relative torsion $\mathcal{R}$ is independent of $g$.

Proposition 4.2 (Hermitian anomaly of the relative torsion)

$$
\begin{gathered}
\log \mathcal{R}\left(\mu_{2}\right)-\log \mathcal{R}\left(\mu_{1}\right)=-\int_{M} V\left(\rho, \mu_{1}, \mu_{2}\right) e(M, g)+ \\
+\sum_{x \in C r(h)}(-1)^{\text {ind } \mathrm{x}} V\left(\rho, \mu_{1}, \mu_{2}\right)(x) .
\end{gathered}
$$

In the case $\mathcal{A}=\mathbb{C}$ these results were established in by Bismut Zhang, cf [BZ]. Propositions 4.1 and 4.2 will be proved at the same time. Their proof is reduced to some local index type results established in [BZ].

Remark Theorem 2.1. can be also derived as a special case of a (slightly more general) version of Proposition 4.2(i).

Proof of Propositions 4.1, 4.2: (ii) follows from (i) by noting that $e(M, g)=$ 0 and $e_{C S}\left(M, g_{1}, g_{2}\right)=0$ if $\operatorname{dim} M$ is odd and that $\theta(\mu)=0$ if the Hermitian structure $\mu$ is parallel with respect to the canonical flat connection.

To prove (i), consider a smooth 1-parameter family $g(u)$ of Riemannian metrics and a smooth 1-parameter family $\mu(u)$ of Hermitian structures, $(-1<u<+1)$. 
We want to compute $\frac{d}{d u} \log \mathcal{R}\left(g(u), \mu_{0}\right)$ and $\frac{d}{d u} \log \mathcal{R}\left(g_{0}, \mu(u)\right)$. We begin by analyzing $\frac{d}{d u} \log \mathcal{R}(g(u), \mu(u))$.

Denote by $\langle\langle\cdot, \cdot\rangle\rangle(u)$ the scalar product defined by $g(u)$ and $\mu(u)$ on $\Omega^{k}(M ; \mathcal{E})$ ) (cf. 2.2) and by $\langle\langle\cdot, \cdot\rangle\rangle_{s}(u)$ the one given by $(2.4)$. Clearly $\langle\langle\cdot, \cdot\rangle\rangle(u)=\langle\langle\cdot, \cdot\rangle\rangle_{0}(u)$.

Denote by $\Delta_{k}(u): \Omega^{k}(M ; \mathcal{E}) \rightarrow \Omega^{k}(M ; \mathcal{E})$ the Laplacian on $\Omega^{k}(M ; \mathcal{E})$ induced by $g(u)$ and $\mu(u)$ and by $H_{s}(u)(\Lambda(M ; \mathcal{E}))$ the completion of $\Omega(M ; \mathcal{E})$ with respect to $\langle\langle\cdot, \cdot\rangle\rangle_{s}(u)$.

Consider the following commutative diagram

$$
\begin{array}{ccc}
H_{0}(u)(\Lambda(M ; \mathcal{E})) & \stackrel{\gamma_{s+s^{\prime}}(u)}{\longrightarrow} & \mathcal{C}(\tau)(u) \\
\downarrow \varphi_{s, s^{\prime}}(u) & & \downarrow \iota(u) \\
H_{0}(0)(\Lambda(M ; \mathcal{E})) & \gamma_{s}(0) & \\
& & \mathcal{C}(\tau)(0)
\end{array}
$$

where

$\gamma_{s}(u):=\operatorname{Int}_{s}(\operatorname{Id}+\Delta(u))^{-s / 2}, \varphi_{s, s^{\prime}}(u):=(\operatorname{Id}+\Delta(0))^{+s / 2}(\operatorname{Id}+\Delta(u))^{-\frac{s+s^{\prime}}{2}}$, and $\iota(u)$ is the identity map on $\mathcal{C}(\tau)$ regarded as an isomorphism of $\mathcal{C}(\tau)$ with the scalar product induced from $\mu(u)$ and $\mathcal{C}(\tau)$ with the scalar product induced by $\mu(0)$.

We recall from subsection 2.1 that, for $s$ and $s^{\prime}$ sufficiently large, $\gamma_{s}(u)$ and $\varphi_{s, s^{\prime}}(u)$ are morphisms of trace class. Since $\gamma_{s+s^{\prime}}(u)$ and $\gamma_{s}(0)$ induce isomorphisms in algebraic cohomology (cf. Proposition 2.5), so does $\varphi_{s, s^{\prime}}(u)$.

We thus can apply Proposition 1.15 to obtain

$$
\log T\left(\mathcal{C}(\iota(u))+\log \mathcal{R}(g(u))=\log \mathcal{R}(g(0))+\log T\left(\mathcal{C}\left(\varphi_{s, s^{\prime}}(u)\right)\right.\right.
$$

where $\mathcal{C}\left(\varphi_{s, s^{\prime}}(u)\right)$ denotes the mapping cone associated to $\varphi_{s, s^{\prime}}(u)$.

Since $(\operatorname{Id}+\Delta(u))^{s / 2}: H_{s}(u)(\Lambda(M ; \mathcal{E})) \rightarrow H_{0}(u)(\Lambda(M ; \mathcal{E}))$ is an isometry, we conclude from Proposition 1.16 that

$$
T\left(\mathcal{C}\left(\varphi_{s, s^{\prime}}(u)\right)=T\left(\mathcal{C}\left(\operatorname{In}_{s+s^{\prime} ; s}(u)\right)\right.\right.
$$

where

$$
\operatorname{In}_{s+s^{\prime}, s}(u): H_{s+s^{\prime}}(u)(\Lambda(M ; \mathcal{E})) \rightarrow H_{s}(0)(\Lambda(M ; \mathcal{E}))
$$

denotes the canonical inclusion. To analyze the torsion of the mapping cone $\left(\mathcal{C}_{u}, D\right):=\left(\mathcal{C}\left(\operatorname{In}_{s+s^{\prime} ; s}(u)\right), d\left(\operatorname{In}_{s+s^{\prime}, s}(u)\right)\right)$, note that

$$
\begin{aligned}
\mathcal{C}_{u k} & :=H_{s}(0)\left(\Lambda^{k-1}(M ; \mathcal{E})\right) \oplus H_{s+s^{\prime}}(u)\left(\Lambda^{k}(M ; \mathcal{E})\right) \\
D_{k} & =\left(\begin{array}{cc}
-d_{k-1} & \operatorname{In}_{s+s^{\prime}, s}(u) \\
0 & d_{k}
\end{array}\right)
\end{aligned}
$$


with inner product $\left(\omega_{1}, \omega_{1}^{\prime} \in \Omega^{k-1}, \omega_{2}, \omega_{2}^{\prime} \in \Omega^{k}\right)$

(4.6)

$$
\left\langle\left\langle\left(\omega_{1}, \omega_{2}\right),\left(\omega_{1}^{\prime}, \omega_{2}^{\prime}\right)\right\rangle\right\rangle:=\left\langle\left\langle\omega_{1}, \omega_{1}^{\prime}\right\rangle\right\rangle_{s}(0)+\left\langle\left\langle\omega_{2}, \omega_{2}^{\prime}\right\rangle\right\rangle_{s+s^{\prime}}(u) .
$$

In order to calculate $\frac{d}{d u} \log T(\mathcal{C}(u))$, introduce the zeroth order differential operators $A_{k}(0): \Omega^{k}(M ; \mathcal{E}) \rightarrow \Omega^{k}(M ; \mathcal{E})$ defined by

$$
\left\langle\left\langle\omega, \omega^{\prime}\right\rangle\right\rangle_{0}(u)=\left\langle\left\langle A_{k}(u) \omega, \omega^{\prime}\right\rangle\right\rangle_{0}(0)
$$

and consider the zeroth order pseudo-differential operator

$$
B_{k}\left(s+s^{\prime} ; u\right): H_{s+s^{\prime}}(0)\left(\Lambda^{k}(M ; \mathcal{E})\right) \rightarrow H_{s+s^{\prime}}(0)\left(\Lambda^{k}(M ; \mathcal{E})\right)
$$

defined by

$B_{k}(u) \equiv B_{k}\left(s+s^{\prime}, u\right):=\left(\operatorname{Id}+\Delta_{k}(u)\right)^{-\left(s+s^{\prime}\right)} A_{k}(u)^{-1} \frac{d}{d u}\left(A_{k}(u)\left(\operatorname{Id}+\Delta_{k}(u)\right)^{s+s^{\prime}}\right)$

so that the following identity holds

$$
\frac{d}{d u}\left\langle\left\langle\omega, \omega^{\prime}\right\rangle\right\rangle_{s+s^{\prime}}(u)=\left\langle\left\langle B_{k}(u) \omega, \omega^{\prime}\right\rangle\right\rangle_{s+s^{\prime}}(0) .
$$

Let $\mathcal{B}_{k}\left(s+s^{\prime}, u\right)$ be the bounded operator in $\mathcal{L}\left(H_{s}(0)\left(\Lambda^{k}(M ; \mathcal{E})\right) \oplus H_{s+s^{\prime}}(0)\left(\Lambda^{k}(M ; \mathcal{E})\right)\right)$ defined by

$$
\mathcal{B}_{k}(u) \equiv \mathcal{B}_{k}\left(s+s^{\prime}, u\right):=\left(\begin{array}{cc}
0 & 0 \\
0 & B_{k}\left(s+s^{\prime}, u\right)
\end{array}\right) .
$$

Then we have, in view of (4.6),

$$
\frac{d}{d u}\left\langle\left\langle\left(\omega_{1}, \omega_{2}\right),\left(\omega_{1}^{\prime}, \omega_{2}^{\prime}\right)\right\rangle\right\rangle=\left\langle\left\langle\mathcal{B}_{k}(u)\left(\omega_{1}, \omega_{2}\right),\left(\omega_{1}^{\prime}, \omega_{2}^{\prime}\right)\right\rangle\right\rangle .
$$

Proceeding as in [BFKM, Appendix 3], one obtains (cf. [BFKM, A3.10]) in view of the fact that the complex $\mathcal{C}_{u}$ is algebraically acyclic,

$$
\frac{d}{d u} \log T\left(\mathcal{C}_{u}\right)=-F p_{z=0} \frac{1}{\Gamma(z)} \int_{0}^{1} x^{z-1} \frac{1}{2} \sum_{q=0}^{n}(-1)^{q} \operatorname{Tr}\left(\mathcal{B}_{q}(u) e^{-x \tilde{\Delta}_{q}(u)}\right) d x
$$

where $\tilde{\Delta}_{q}(u)$ denotes the $q$-Laplacian of $\mathcal{C}_{u}$ with respect to the inner product (4.6).

By formula (1.23),

$$
\tilde{\Delta}_{q}(u)=\left(\begin{array}{cc}
\Delta_{q-1}(0)+\psi_{q-1}(u) & \eta_{q}(u) \\
\eta_{q}(u)^{*} & \Delta_{q}(u)+\psi_{q}(u)
\end{array}\right)
$$

where $\left(\begin{array}{cc}\psi_{q-1}(u) & \eta_{q}(u) \\ \eta_{q}(u)^{*} & \psi_{q}(u)\end{array}\right)$ is a nonnegative selfadjoint operator of trace class $\left(s, s^{\prime}\right.$ sufficiently large). 
By the remark after Proposition 1.5 we conclude, in view of definitions (1.5) and (1.7),

$$
\begin{aligned}
& -\frac{1}{2} F p_{z=0} \frac{1}{\Gamma(z)} \int_{0}^{1} x^{z-1} \sum_{q=0}^{n}(-1)^{q} \operatorname{Tr}\left(\mathcal{B}_{q}(u) e^{-x \tilde{\Delta}_{q}(u)}\right) d x \\
= & -\frac{1}{2} F p_{z=0} \frac{1}{\Gamma(z)} \int_{0}^{1} x^{z-1} \sum_{q=0}^{n}(-1)^{q} \operatorname{Tr}\left(B_{q}(u) e^{-x \Delta_{q}(u)}\left(\mathrm{Id}-P_{q ; u}\right)\right) d x \\
& +\frac{1}{2} \sum_{q=0}^{n}(-1)^{q} \operatorname{Tr}\left(B_{q}(u) P_{q ; u}\right) \\
= & -\frac{1}{2} F p_{z=0} \frac{1}{\Gamma(z)} \int_{0}^{1} x^{z-1} \sum_{q=0}^{n}(-1)^{q} \operatorname{Tr}\left(B_{q}(u) e^{-x \Delta_{q}(u)}\right) d x
\end{aligned}
$$

where $P_{q ; u} \equiv P_{q ; u}(\{0\})$ is the orthogonal projector onto the null space of $\Delta_{q}(u)$. Introduce

$$
\begin{aligned}
a(x ; u) & :=\sum_{q=0}^{n}(-1)^{q} \operatorname{Tr}\left(B_{q}(s ; u) e^{-x \Delta_{q}(u)}\right) \\
& =\sum_{q=0}^{n}(-1)^{q} \operatorname{Tr}\left(\left(\operatorname{Id}+\Delta_{q}(u)\right)^{-s}\right. \\
& \left.A_{q}(u)^{-1} \frac{d}{d u}\left(A_{q}(u)\left(\operatorname{Id}+\Delta_{q}(u)\right)^{s}\right) e^{-x \Delta_{q}(u)}\right) .
\end{aligned}
$$

To investigate the right hand side of (4.8) introduce

$$
b(x ; u):=\sum_{q=0}^{n}(-1)^{q} \operatorname{Tr}\left(A_{q}(u)^{-1}\left(\frac{d}{d u} A_{q}(u)\right) e^{-x \Delta_{q}(u)}\right) .
$$

According to [BZ],

$$
F p_{z=0} \frac{1}{\Gamma(z)} \int_{0}^{1} x^{z-1} b(x ; u) d x
$$

is, in the case where $\mu(u)=\mu$ is constant, given by

$$
-\int_{M} \theta(\rho, \mu) \wedge \frac{d}{d u} e_{C S}(M, g(0), g(u))
$$

and, when $g(u)=g$ is constant by

$$
\int_{M} \frac{d}{d u} V(\rho, \mu(u), \mu(0)) e(M, g) .
$$

Actually, in [BZ], these formulae are proven only for the case $\mathcal{A}=\mathbb{C}$, but the same arguments work "word by word" for $\mathcal{A}$ arbitrary. In this paper $\theta(\rho, \mu)$ is defined to be $1 / 2$ of the corresponding quantity in [BZ]. 
We will show that

$$
a(x ; u)=b(x ; u) .
$$

From the equality

$$
\frac{d}{d u}\left(\left(\operatorname{Id}+\Delta_{q}(u)\right)^{s}\left(\operatorname{Id}+\Delta_{q}(u)\right)^{-s}\right)=0
$$

and the commutativity of the trace, $\operatorname{Tr} A B=\operatorname{Tr} B A$, we obtain

$$
\begin{aligned}
& \operatorname{Tr}\left(\left(\operatorname{Id}+\Delta_{q}(u)\right)^{-s} A_{q}(u)^{-1} \frac{d}{d u}\left(A_{q}(u)\left(\operatorname{Id}+\Delta_{q}(u)\right)^{s}\right) e^{-x \Delta_{q}(u)}\right) \\
& =\operatorname{Tr}\left(A_{q}(u)^{-1}\left(\frac{d}{d u} A_{q}(u)\right) e^{-x \Delta_{q}(u)}\right) \\
& \quad+\operatorname{Tr}\left(\left(\operatorname{Id}+\Delta_{q}(u)\right)^{-s} \frac{d}{d s}\left(\left(\operatorname{Id}+\Delta_{q}(u)\right)^{-s}\right) e^{-x \Delta_{q}(u)}\right) \\
& =\operatorname{Tr}\left(A_{q}(u)^{-1}\left(\frac{d}{d u} A_{q}(u)\right) e^{-x \Delta_{q}(u)}\right)+ \\
& \quad+s \operatorname{Tr}\left(\left(\operatorname{Id}+\Delta_{q}(u)\right)^{-1} \frac{d}{d u}\left(\Delta_{q}(u)\right) e^{-x \Delta_{q}(u)}\right) \\
& =\operatorname{Tr}\left(A_{q}(u)^{-1} \frac{d A_{q}(u)}{d u} e^{-x \Delta_{q}(u)}\right)+s \frac{d}{d u} \operatorname{Tr} f\left(\Delta_{q}(u)\right)
\end{aligned}
$$

where $f(x, y):=-\int_{y}^{\infty} \frac{e^{-x r}}{(1+r)} d r$.

Since $\Delta_{q}(u)$ is selfadjoint and nonnegative one obtains, for $x>0$,

$$
\left\|f\left(x, \Delta_{q}(u)\right)\right\|_{t r}:=\int_{0}^{\infty} \lambda|f(x, \lambda)| d N_{\Delta_{q}(u)} \leq \int_{0}^{\infty} \lambda e^{-\lambda x} d N_{\Delta_{q}(u)}(\lambda)<\infty .
$$

Therefore, $f\left(x, \Delta_{q}(u)\right)$ is of trace class for $x>0$ and

$$
\begin{aligned}
\sum_{q=0}^{n}(-1)^{q} \operatorname{Tr} f\left(x, \Delta_{q}(u)\right) & =\sum_{q=0}^{n}(-1)^{q} \operatorname{Tr} f\left(x, \Delta_{q}^{+}(u)\right)+ \\
& +\sum_{q=0}^{n}(-1)^{q} \operatorname{Tr} f\left(x, \Delta_{q}^{-}(u)\right) \\
& =\sum_{q=1}^{n}(-1)^{q}\left(\operatorname{Tr} f\left(x, \Delta_{q}^{+}(u)\right)-\operatorname{Tr} f\left(x, \Delta_{q-1}^{-}\right)\right) \\
& =0
\end{aligned}
$$

where we used that $\Delta_{q}^{+}(u)$ and $\Delta_{q}^{-}(u)$ are isospectral and, therefore,

$$
\operatorname{Tr} f\left(x, \Delta_{q}^{+}(u)\right)=\operatorname{Trf}\left(x, \Delta_{q}^{-}(u)\right) .
$$


Thus, for any $x \geq 0$,

$$
\begin{aligned}
a(x ; u) & =b(x ; u)+s \frac{d}{d u} \sum_{q=0}^{n}(-1)^{q} \operatorname{Tr} f\left(x, \Delta_{q}(u)\right) \\
& =b(x ; u) .
\end{aligned}
$$

Note that by Proposition 1.12

$$
\log T\left(\mathcal{C}(\iota(u))=\sum_{x \in C r h}(-1)^{\text {ind } x} V(\rho, \mu(u), \mu(0))(x) .\right.
$$

Combining these with (4.8)-(4.11), statement (i) in Proposition 4.1 and Proposition 4.2 follow.

\subsection{Proof of Proposition 0.1}

We now prove Proposition 0.1 in full generality. Let $g$ be an arbitrary Riemannian metric of $M$ and $g^{\prime}$ the metric given by the generalized triangulation $\tau$. Denote the corresponding relative torsions by $\mathcal{R}(g)$ and $\mathcal{R}\left(g^{\prime}\right)$. Proposition 4.1 and Proposition 0.1 in the special case considered in section 3 imply Proposition 0.1: Indeed, denote by $\alpha\left(g^{\prime}\right)$ the density given in subsection 3.1 (D) and define $\alpha(g):=\alpha\left(g^{\prime}\right)+\theta \wedge e_{C S}\left(g, g^{\prime}\right)$ Clearly, $\alpha$ is a local quantity and satisfies (cf subsection 3.1 (B) and (D)) $\log \mathcal{R}(g)=\int_{M \backslash C r(h)} \alpha(g)$. Further $\alpha(g)$ vanishes if $\mu$ is parallel (cf subsection 3.1 (D) and Proposition 4.1(ii)).

\subsection{Anomaly with respect to triangulations}

In this subsection we investigate how the relative torsion depends on the triangulation.

Suppose $\tau_{1}=\left(h_{1}, g_{1}\right)$ and $\tau_{2}=\left(h_{2}, g_{2}\right)$ are two generalized triangulation which admit a common subdivision $\tau_{0}=\left(h_{0}, g_{0}\right)$. Recall from [BFKM, Subsection 6.3] that $\tau_{0}$ is called a subdivision of $\tau_{1}$ if :

$$
\text { (i) } C r_{q}\left(h_{1}\right) \subseteq C r_{q}\left(h_{0}\right),(0 \leq q \leq n), W_{x}^{-}\left(\tau_{0}\right) \subseteq W_{x}^{-}\left(\tau_{1}\right),\left(x \in C r\left(h_{1}\right)\right)
$$

and for any $y \in C r\left(h_{1}\right)$

$$
\text { (ii) } W_{y}^{-}\left(\tau_{1}\right)=\cup_{x \in C r\left(h_{0}\right), x \in W_{y}^{-}\left(\tau_{1}\right)} W_{x}^{-}\left(\tau_{0}\right),
$$

where $C r_{q}\left(h_{i}\right)$ denotes the set of critical points of index $q$ of $h_{i}(i=0,1,2)$, $W_{x}^{-}\left(\tau_{1}\right)$ denotes the unstable manifold of $x \in C r\left(h_{1}\right)$ with respect to the gradient flow $-\operatorname{grad}_{g_{1}}\left(h_{1}\right)$ and $g_{0}=g_{1}, h_{0}=h_{1}$ in a neighborhood of the critical points of $h_{1}$. 
Given $x \in C r\left(h_{0}\right)$, there exist unique elements $x_{1} \in \operatorname{Cr}\left(h_{1}\right), x_{2} \in C r\left(h_{2}\right)$ so that $x \in W_{x_{j}}^{-}\left(\tau_{j}\right)(j=1,2)$.

Introduce the function $w=w_{\tau_{0}}=w\left(\tau_{1}, \tau_{2} ; \tau_{0}\right): C r\left(h_{0}\right) \rightarrow \mathbb{R}$ by setting

$$
w(x):=\log \operatorname{vol}\left(T_{x, x_{2}}^{\tau_{2}} \circ\left(T_{x, x_{1}}^{\tau_{1}}\right)^{-1}\right)
$$

where $T_{x, x_{j}}^{\tau_{j}}: \mathcal{E}_{x} \rightarrow \mathcal{E}_{x_{j}}(j=1,2)$ denotes the parallel transport along an arbitrary curve in $W_{x_{j}}^{-}\left(\tau_{j}\right)$ joining $x$ and $x_{j}$. Define $\omega_{\tau_{1}, \tau_{2}}=\omega\left(\tau_{1}, \tau_{2} ; \tau_{0}\right)$ by

$$
\omega_{\tau_{1}, \tau_{2}}:=\sum_{x \in C r\left(h_{0}\right)}(-1)^{\operatorname{index}(x)} w(x) .
$$

Notice that $\omega\left(\tau_{1}, \tau_{2} ; \tau_{0}\right)$ is independent of the choice of $\tau_{0}$, i.e.,

$$
\omega\left(\tau_{1}, \tau_{2} ; \tilde{\tau}_{0}\right)=\omega\left(\tau_{1}, \tau_{2} ; \tau_{0}\right)
$$

for any generalized triangulation $\tilde{\tau}_{0}=\left(\tilde{h}_{0}, \tilde{g}_{0}\right)$ which is a subdivision of $\tau_{0}$. To see it, notice that for any $y \in \operatorname{Cr}\left(\tilde{h}_{0}\right)$, there exists a unique $x \in \operatorname{Cr}\left(h_{0}\right)$ with $y \in W_{x}^{-}\left(\tau_{0}\right)$. Use that $T_{y, x_{j}}^{\tau_{j}}=T_{x, x_{j}}^{\tau_{j}} T_{y, x}^{\tau_{0}}(j=1,2)$ to conclude that $w_{\tilde{\tau}_{0}}(y)=w_{\tau_{0}}(x)$ and thus

$$
\sum_{y \in C r\left(\tilde{h}_{0}\right) \cap W_{x}^{-}\left(\tau_{0}\right)}(-1)^{\operatorname{index}(y)} w_{\tilde{\tau}_{0}}(y)=w_{\tau_{0}}(x) \sum_{y \in C r\left(\tilde{h}_{0}\right) \cap W_{x}^{-}\left(\tau_{0}\right)}(-1)^{\operatorname{index}(y)} .
$$

As $\tilde{\tau}_{0}$ is a subdivision of $\tau_{0}$

$$
\sum_{\substack{y \neq x \\ y \in C r\left(\tilde{h}_{0}\right) \cap W_{x}^{-}\left(\tau_{0}\right)}}(-1)^{\operatorname{index}(y)}=0
$$

we conclude that $\omega\left(\tau_{1}, \tau_{2} ; \tilde{\tau}_{0}\right)=\omega\left(\tau_{1}, \tau_{2} ; \tau_{0}\right)$. Moreover, if $\tau_{1}, \tau_{2}$ and $\tau_{3}$ are three generalized triangulation which admit a common subdivision $\tau_{0}$, then

$$
\omega_{\tau_{1}, \tau_{2}}+\omega_{\tau_{2}, \tau_{3}}=\omega_{\tau_{1}, \tau_{3}} .
$$

Proposition 4.3 Assume that the generalized triangulations $\tau_{1}$ and $\tau_{2}$ admit a common subdivision $\tau_{0}$. Then

(i) $\log \mathcal{R}\left(\tau_{1}\right)-\log \mathcal{R}\left(\tau_{2}\right)=\omega_{\tau_{1}, \tau_{2}}$

(ii) If $\mu$ is parallel with respect to the canonical flat connection of $\mathcal{E}$, then $\mathcal{R}\left(\tau_{1}\right)=\mathcal{R}\left(\tau_{2}\right)$ provided $\tau_{1}$ and $\tau_{2}$ have a common subdivision.

Proof: Statement (ii) follows from (i) by noticing that $w(x)=0, \forall x \in C r\left(h_{0}\right)$ and thus $\omega_{\tau_{1}, \tau_{2}}=0$.

To prove (i), notice that

$$
\log \mathcal{R}\left(\tau_{2}\right)-\log \mathcal{R}\left(\tau_{1}\right)=\left(\log \mathcal{R}\left(\tau_{2}\right)-\log \mathcal{R}\left(\tau_{0}\right)\right)+\left(\log \mathcal{R}\left(\tau_{0}\right)-\log \mathcal{R}\left(\tau_{1}\right)\right)
$$


In view of (4.16), it suffices to consider the case where $\tau_{2}=\tau_{0}$. The subdivision $\tau_{2}$ can be obtained from $\tau_{1}$ by a sequence $\tau_{1}=\sigma_{1}, \ldots, \sigma_{N}=\tau_{2}$, where $\sigma_{j+1}=\left(h_{j+1}, g_{j+1}^{\prime}\right)$ is a subdivision of $\sigma_{j}=\left(h_{j}, g_{j}^{\prime}\right)$ with $\operatorname{Cr}\left(h_{j+1}\right)=$ $C r\left(h_{j}\right) \cup\left\{x_{j+1}, y_{j+1}\right\}$ so that there exists $z_{j} \in C r\left(h_{j}\right)$ with $x_{j+1}, y_{j+1} \in W_{z_{j}}^{-}$ and index $\left(x_{j+1}\right)=\operatorname{index}\left(z_{j}\right)=\operatorname{index}\left(y_{j+1}\right)+1$. Recall that $W_{x}^{-} \equiv W_{x ; h_{j}}^{-}$ denotes the unstable manifold, associated to $x \in C r\left(h_{j}\right)$ and the gradient flow $-\operatorname{grad}_{g_{j}} h_{j}$. In view of (4.15), it suffices to consider the case where $\tau_{2}=\sigma_{2}$. To ease notation, we write $\tau:=\tau_{1}=\left(h_{1}, g_{1}^{\prime}\right), \sigma:=\tau_{2}=\left(h_{2}, g_{2}^{\prime}\right) z:=z_{1}, x^{\prime}:=x_{2}$, $y^{\prime}:=y_{2}$. Then, with $q_{0}=\operatorname{index}(z)$

$$
C r_{q}\left(h_{2}\right)= \begin{cases}C r_{q}\left(h_{1}\right) & q \neq q_{0}, q_{0}-1 \\ C r_{q}\left(h_{1}\right) \cup\left\{y^{\prime}\right\} & q=q_{0}-1 \\ C r_{q}\left(h_{1}\right) \cup\left\{x^{\prime}\right\} & q=q_{0}\end{cases}
$$

Consider the following commutative diagram

$$
\begin{array}{ccc}
H_{s}(\Lambda(M, \mathcal{E})) & \stackrel{\mathrm{Int}_{s}(\tau)}{\longrightarrow} & \mathcal{C}(\tau) \\
\operatorname{Int}_{s}(\sigma) \searrow & & \nearrow A \\
& & \\
& \mathcal{C}(\sigma) &
\end{array}
$$

where $A_{q}(0 \leq q \leq n)$ is defined as follows: for a section $s \in C^{\infty}\left(\left.\mathcal{E}\right|_{C r_{q} h_{2}}\right)$ and a critical point $x \in C r_{q}\left(h_{1}\right)$, set

$$
A_{q}(s)(x):=\sum_{y \in C r_{q}\left(h_{2}\right) \cap W_{x}^{-}} T_{y x}(s(y))
$$

where $T_{y x}: \mathcal{E}_{y} \rightarrow \mathcal{E}_{x}$ denotes the parallel transport from $y$ to $x$ along a curve in $W_{x}^{-}=W_{x}^{-}(\tau)$. The map $A$ is a morphism of cochain complexes of $\mathcal{A}$-Hilbert modules of finite type which induces an isomorphism in algebraic cohomology. As Int ${ }_{s}$ and $A$ are of trace class, we then conclude from Proposition 1.15 that

$$
\log \mathcal{R}(\tau)=\log \mathcal{R}(\sigma)+\log T(\mathcal{C}(A)) .
$$

Thus the claimed result follows once we show that

$$
\log T(\mathcal{C}(A))=\omega_{\sigma \tau} .
$$

Formula (4.17) can be verified, using a localization argument: Consider first the case where $\mu$ is parallel. Clearly, $\omega_{\sigma \tau}=0$. On the other hand, $\log \mathcal{R}(\tau)=$ $\log \mathcal{R}(\sigma)=0$ (Proposition 0.1 (ii)) and thus $\log T(\mathcal{C}(A))=\log \mathcal{R}(\tau)-\log \mathcal{R}(\sigma)=$ 0 . Hence (4.17) holds in this case.

Further, if $\mathcal{E}$ admits a Hermitian structure $\mu_{0}$ which is parallel, then 
(4.17) remains valid. Indeed, consider the following commutative diagram

$$
\begin{aligned}
& \mathcal{C}(\sigma, \mu) \stackrel{A}{\longrightarrow} \mathcal{C}(\tau, \mu) \\
& \downarrow \operatorname{Id}_{\sigma, \mu, \mu_{0}} \quad \downarrow \operatorname{Id}_{\tau, \mu, \mu_{0}} \\
& \mathcal{C}\left(\sigma, \mu_{0}\right) \stackrel{A}{\longrightarrow} \mathcal{C}\left(\tau, \mu_{0}\right)
\end{aligned}
$$

Then, according to Proposition 1.12,

$$
\log T\left(\mathcal{C}\left(\operatorname{Id}_{\sigma, \mu, \mu_{0}}\right)\right)+\log T\left(\mathcal{C}\left(A, \mu_{0}\right)\right)=\log T(\mathcal{C}(A, \mu))+\log T\left(\mathcal{C}\left(\operatorname{Id}_{\tau, \mu, \mu_{0}}\right)\right) .
$$

By Proposition 1.14, $(j=1,2)$

$$
\begin{aligned}
\log T\left(\mathcal{C}\left(\operatorname{Id}_{\sigma, \mu, \mu_{0}}\right)\right)- & \log T\left(\mathcal{C}\left(\operatorname{Id}_{\tau, \mu, \mu_{0}}\right)\right) \\
= & \sum_{q}(-1)^{q} \log \operatorname{vol}\left(\operatorname{Id}_{q ; \sigma, \mu, \mu_{0}}\right) \\
& -\sum_{q}(-1)^{q} \log \operatorname{vol}\left(\operatorname{Id}_{q, \tau, \mu, \mu_{0}}\right) \\
= & \sum_{y \in C r\left(h_{2}\right)}(-1)^{\operatorname{index}(y)} \log \operatorname{vol}\left(\operatorname{Id}_{y, \mu, \mu_{0}}\right) \\
& -\sum_{y \in C r\left(h_{1}\right)}(-1)^{\operatorname{index}(y)} \log \operatorname{vol}\left(\operatorname{Id}_{y, \mu, \mu_{0}}\right) \\
= & (-1)^{\operatorname{index}\left(x^{\prime}\right)} \log \operatorname{vol}\left(\operatorname{Id}_{x^{\prime}, \mu, \mu_{0}}\right) \\
& +(-1)^{\operatorname{index}\left(y^{\prime}\right)} \log \operatorname{vol}\left(\operatorname{Id}_{y^{\prime}, \mu, \mu_{0}}\right) .
\end{aligned}
$$

Combining the above equalities with $\log T\left(\mathcal{C}\left(A, \mu_{0}\right)\right)=0$ we obtain

$$
\begin{gathered}
\log T(\mathcal{C}(A, \mu))=(-1)^{\operatorname{index}\left(x^{\prime}\right)} \log \operatorname{vol}\left(\operatorname{Id}_{x^{\prime}, \mu, \mu_{0}}\right)+ \\
+(-1)^{\operatorname{index}\left(y^{\prime}\right)} \log \operatorname{vol}\left(\operatorname{Id}_{y^{\prime}, \mu, \mu_{0}}\right) .
\end{gathered}
$$

Observe that

$$
\log \operatorname{vol}\left(\operatorname{Id}_{x^{\prime}, \mu, \mu_{0}}\right)=\log \operatorname{vol}_{\mu}\left(T_{x^{\prime}, z^{\prime}}\right)+\log \operatorname{vol}\left(\operatorname{Id}_{z^{\prime}, \mu, \mu_{0}}\right)
$$

and, similarly,

$$
\log \operatorname{vol}\left(\operatorname{Id}_{y^{\prime}, \mu, \mu_{0}}\right)=\log \operatorname{vol}_{\mu}\left(T_{y^{\prime}, x_{0}}\right)+\log \operatorname{vol}\left(\operatorname{Id}_{z^{\prime}, \mu, \mu_{0}}\right) .
$$

As index $\left(x^{\prime}\right)=\operatorname{index}\left(y^{\prime}\right)+1$, this leads to

$$
\begin{aligned}
\log T(\mathcal{C}(A, \mu)) & =(-1)^{\operatorname{index}\left(x^{\prime}\right)} \log \operatorname{vol}_{\mu}\left(T_{x^{\prime} z^{\prime}}\right) \\
& +(-1)^{\operatorname{index}\left(y^{\prime}\right)} \log \operatorname{vol}_{\mu}\left(T_{y^{\prime} z^{\prime}}\right) \\
& =\omega_{z \tau}
\end{aligned}
$$


which establishes (4.17) in the case where $\mathcal{E}$ admits a Hermitian structure which is parallel.

Now use a standard localization to conclude that (4.17) is true in general.

\section{Proof of Theorem 0.1}

\subsection{Proof of Theorem 0.1}

Consider the system $\mathcal{F}:=(M, \rho, \mu, g, \tau) \equiv(M, \mathcal{E}, \nabla, \mu, g, \tau)$, with $\tau=\left(h, g^{\prime}\right)$ and denote by $X$ the vector field $X:=-\operatorname{grad}_{g^{\prime}} h . X$ defines a smooth map $X: M \backslash C r(h) \rightarrow T M \backslash M$. Denote by

$\beta_{\mathcal{F}} \equiv \beta(M, \rho, \mu, g, \tau):=(-1)^{n+1} \theta(\rho, \mu) \wedge X^{*}(\Psi(T M, g)) \in \Omega^{n}\left(M \backslash C r(h) ; \mathcal{O}_{\mathcal{M}}\right)$.

Observe that if $\mu$ is parallel in a neighborhood of $C r(h)$ both $\alpha \equiv \alpha_{\mathcal{F}}$, by Proposition 3.2 (iii), and $\beta_{\mathcal{F}}$ vanish in the neighborhood of $C r(h)$.

Proposition 3.2 (ii) and the equalities (4.1) and (4.2 ${ }^{v}$ ) imply that for $\gamma(M, \rho, \mu, g, \tau)=\alpha(M, \rho, \mu, g, \tau)$ or $\beta(\mathrm{M}, \rho, \mu, \mathrm{g}, \tau)$ we have

(5.1) $\quad \gamma\left(M_{1} \times M_{2}, \rho_{1} \otimes \rho_{2}, g_{1} \times g_{2}, \mu_{1} \otimes \mu_{2}, \tau_{1} \times \tau_{2}\right)=$ $\gamma\left(M_{1}, \rho_{1}, g_{1}, \mu_{1}, \tau_{1}\right) e\left(M_{2}, g_{2}\right) \operatorname{dim}\left(\mathcal{W}_{2}\right)+\gamma\left(\mathrm{M}_{2}, \rho_{2}, \mathrm{~g}_{2}, \mu_{2}, \tau_{2}\right) \mathrm{e}\left(\mathrm{M}_{1}, \mathrm{~g}_{1}\right) \operatorname{dim}\left(\mathcal{W}_{1}\right)$.

Choose a Hermitian structure $\mu_{0}$ which is parallel in a neighborhood of $\mathrm{Cr}(h)$. Introduce the quantity

$$
\begin{array}{r}
\mathcal{S}\left(M, \rho, \mu, \mu_{0}, g, \tau\right):=\log \mathcal{R}(M, \rho, \mu, g, \tau)-\int_{M \backslash C r(h)} \beta\left(M, \rho, \mu_{0}, g, \tau\right)- \\
-\int_{M} V\left(\rho, \mu, \mu_{0}\right) e(M, g)+\sum_{x \in C r(h)}(-1)^{\operatorname{ind}(\mathrm{x})} V\left(\rho, \mu, \mu_{0}\right)(x)
\end{array}
$$

The proof of Theorem 0.1 uses the following four statements:

(A) $\mathcal{S}\left(M, \rho, \mu, \mu_{0}, g, \tau\right)$ is independent of $\mu, \mu_{0}, g, \tau$.

Therefore we write $\mathcal{S}(M, \rho)$ instead of $\mathcal{S}\left(M, \rho, \mu, \mu_{0}, g, \tau\right)$.

(B) If $\rho$ is isomorphic to its dual, then $\mathcal{S}(M, \rho)+(-1)^{n+1} \mathcal{S}(M, \rho)=0$.

(C) $\mathcal{S}(M, \rho)=(-1)^{n+1} \mathcal{S}\left(M, \rho^{\sharp}\right)$ where $\rho^{\sharp}$ is the dual representation .

(D) $\mathcal{S}\left(M_{1} \times M_{2}, \rho_{1} \otimes \rho_{2}\right)=\mathcal{S}\left(M_{1}, \rho_{1}\right) \chi\left(M_{2}\right) \operatorname{dim} \mathcal{W}_{1}+\chi\left(\mathrm{M}_{1}\right) \mathcal{S}\left(\mathrm{M}_{2}, \rho_{2}\right) \operatorname{dim} \mathcal{W}_{2}$.

(A) follows from Propositions 4.1-4.3. and the formulas 4.1"-4.1"' and 4.2'-4.2"'. Precisely, the independence of $g$ follows from Proposition 4.1. and formula 4.2", 
and the independence of $\mu$ from Proposition 4.2 and the formulas 4.1" and 4.1"'. To check the independence on $\mu_{0}$ one uses $g=g^{\prime}$ and proceeds as follows: Choose coordinates in a neighborhood of the critical points so that (T3) in the definition of a generalized triangulation is satisfied. Denote by $D_{x}(\epsilon)$ the disc of radius $\epsilon$ and centered at $x \in C r(h)$ and put $D(\epsilon):=\bigsqcup_{x \in C r(h)} D_{x}(\epsilon)$. Let $\tilde{\mu}_{0}$ and $\mu_{0}$ be two Hermitian structures parallel in the neighborhood of the critical points. A straightforward application of the definition of $\mathcal{S}$ combined with $4.1^{\prime}-4.2^{v}$ leads to

$$
\begin{gathered}
\mathcal{S}\left(M, \rho, \mu, \tilde{\mu}_{0}, g^{\prime}, \tau\right)-\mathcal{S}\left(M, \rho, \mu, \mu_{0}, g^{\prime}, \tau\right)= \\
=\lim _{\epsilon \rightarrow 0}\left(( - 1 ) ^ { n } \int _ { M \backslash D ( \epsilon ) } d \left(V\left(\rho, \tilde{\mu}_{0}, \mu_{0}\right) X^{*}\left(\Psi\left(T M, g^{\prime}\right)\right)-\right.\right. \\
\left.-(-1)^{n} \int_{M \backslash D(\epsilon} V\left(\rho, \tilde{\mu}_{0}, \mu_{0}\right) e\left(M, g^{\prime}\right)\right)+ \\
+\int_{M} V\left(\rho, \tilde{\mu}_{0}, \mu_{0}\right) e\left(M, g^{\prime}\right)-\sum_{x \in C r(h)}(-1)^{\text {ind x }}\left(V\left(\rho, \tilde{\mu}_{0}, \mu_{0}\right)(x) .\right.
\end{gathered}
$$

Using Stokes theorem and, when $n$ is odd, the vanishing of $e\left(M, g^{\prime}\right)$ one obtains

$$
\begin{gathered}
\mathcal{S}\left(M, \rho, \mu, \tilde{\mu}_{0}, g^{\prime}, \tau\right)-\mathcal{S}\left(M, \rho, \mu, \mu_{0}, g^{\prime}, \tau\right)= \\
=\lim _{\epsilon \rightarrow 0}\left((-1)^{n} \int_{\partial(M \backslash D(\epsilon))} V\left(\rho, \tilde{\mu}_{0}, \mu_{0}\right) X^{*}\left(\Psi\left(T M, g^{\prime}\right)\right)-\right. \\
-\sum_{x \in C r(h)}(-1)^{\text {ind x }}\left(V\left(\rho, \tilde{\mu}_{0}, \mu_{0}\right)(x)=\right. \\
=\lim _{\epsilon \rightarrow 0}\left((-1)^{n+1} \int_{\partial(D(\epsilon))} V\left(\rho, \tilde{\mu}_{0}, \mu_{0}\right) X^{*}\left(\Psi\left(T M, g^{\prime}\right)\right)-\right. \\
-\sum_{x \in C r(h)}(-1)^{\text {ind x }} V\left(\rho, \tilde{\mu}_{0}, \mu_{0}\right)(x) .
\end{gathered}
$$

Since $V\left(\rho, \tilde{\mu}_{0}, \mu_{0}\right)$ is constant on each $D_{x}(\epsilon)$ one obtains

$$
\begin{gathered}
\mathcal{S}\left(M, \rho, \mu, \tilde{\mu}_{0}, g^{\prime}, \tau\right)-\mathcal{S}\left(M, \rho, \mu, \mu_{0}, g^{\prime}, \tau\right)= \\
=\sum_{x \in C r(h)} V\left(\rho, \tilde{\mu}_{0}, \mu_{0}\right)(x)\left((-1)^{n+1} \int_{\partial D_{x}(\epsilon)} X^{*}\left(\Psi\left(T M, g^{\prime}\right)\right)-(-1)^{\text {ind } \mathrm{x}}\right) .
\end{gathered}
$$

Since in the coordinates we have chosen

$$
X=\sum_{k=1}^{\text {ind } \mathrm{x}} x_{k} \frac{\partial}{\partial x_{k}}-\sum_{k=\text { ind } \mathrm{x}+1}^{n} x_{k} \frac{\partial}{\partial x_{k}}
$$


$4.2^{i v}$ implies that

$$
(-1)^{n+1} \int_{\partial D_{x}(\epsilon)} X^{*}\left(\Psi\left(T M, g^{\prime}\right)\right)=(-1)^{\text {ind } \mathrm{x}}
$$

and therefore

$$
\mathcal{S}\left(M, \rho, \mu, \tilde{\mu}_{0}, g^{\prime}, \tau\right)=\mathcal{S}\left(M, \rho, \mu, \mu_{0}, g^{\prime}, \tau\right)
$$

The independence of $\tau$ can be verified in the following way: One considers a subdivision $\tau^{\prime}$ of $\tau$ so that only those cells of $\tau$, lying in a contractible open set $U$, are subdivided. We choose $\mu_{0}$ to be parallel on $U$ in addition of being parallel in a neighborhood of the critical points of $h$ and let $\mu:=\mu_{0}$. Since $\omega_{\tau, \tau^{\prime}}$ as defined in subsection 4.3 is zero, it follows from Proposition 4.3 that $\mathcal{S}(M, . ., \tau)=\mathcal{S}\left(M, . ., \tau^{\prime}\right)$.

If $\tau_{1}$ and $\tau_{2}$ are two generalized triangulations, one can find a third triangulation, $\tau_{0}$, which is simultaneously a subdivision of $\tau_{1}$ and , up to a composition with a diffeomorphism of $M$ isotopic to the identity, a subdivision of $\tau_{2}$. Since one can pass from $\tau_{i}, i=1,2$ to $\tau_{0}$ by a finite sequence of subdivisions each of them involving subdivisions of cells lying in a contractible open set, the result follows.

To check (B) we set $\mu:=\mu_{0}$ and choose $\mu_{0}$ with $\theta\left(\rho, \mu_{0}\right)=0$. In view of the assumption that $\rho$ is isomorphic to $\rho^{\sharp}$ this is possible (cf subsection 4.1). The result follows then from Proposition 3.2 (i).

To check (C) we choose again $\mu=\mu_{0}$. As $\theta\left(\rho^{\sharp}, \mu_{0}^{\sharp}\right)=-\theta\left(\rho, \mu_{0}\right)$ and $X_{\tau_{\mathcal{D}}}=$ $-\operatorname{grad}_{g^{\prime}}(n-h)=\operatorname{grad}_{g^{\prime}} h=-X_{\tau}$ we conclude in view of $4.2 "$ ' that

$$
\int_{M} \theta\left(\rho, \mu_{0}\right) \wedge X_{\tau}^{*} \Psi+(-1)^{n} \int_{M} \theta\left(\rho^{\sharp}, \mu_{0}^{\sharp}\right) \wedge X_{\mathcal{D}_{\tau}}^{*} \Psi=0 .
$$

Statement $(\mathrm{C})$ follows once we verify that

$$
\int_{M} \alpha(M, \rho, g, \mu, \tau, \epsilon)+(-1)^{n} \int_{M} \alpha\left(M, \rho^{\sharp}, g, \mu^{\sharp}, \tau_{\mathcal{D}}, \epsilon\right)=0 .
$$

With the notation from subsection 3.2 and in view of formula 3.13 and the fact that the $q$-Laplacian corresponding to $(\rho, \mu, g, h)$ is conjugated by the Hodge star operator to the $(n-q)$-Laplacian corresponding to $\left(\rho^{\sharp}, \mu^{\sharp}, g, n-h\right)$, the left hand side of the equality above is $\int_{M} \delta_{\mathcal{F}}(\epsilon)$ with

$$
\delta_{\mathcal{F}}(\epsilon)=\delta_{\mathcal{F}}(h, \epsilon):=n / 2 \sum_{q}(-1)^{q+1} \alpha_{\mathcal{F}}^{q}(h, \epsilon) .
$$

We want to check that $\int_{M} \delta_{\mathcal{F}}(0)=0$ and proceed as in the proof of Proposition 3.2 (iv). Given the system $\mathcal{F}$ one chooses a system $\tilde{\mathcal{F}}$ with the same underlying Riemannian manifold $(M, g)$, the same triangulation $\tau$, but with $\tilde{\rho}$ the trivial representation over the same Hilbert module and with $\tilde{\mu}$ a parallel Hermitian structure. 
In view of 5.2, introduce $W_{\mathcal{F}}(t, \epsilon):=\frac{n}{2} \sum_{q}(-1)^{q} \log \operatorname{det}\left(\Delta_{q}(t)+\epsilon\right)$. By the same arguments as in the proof of Proposition 3.2 (iv) (Mayer Vietoris arguments), we conclude that

$$
F T_{t=\infty}\left(W_{\mathcal{F}}(t, \epsilon)-W_{\tilde{\mathcal{F}}}(t, \epsilon)\right)=\int_{M \backslash C r(h)} \delta_{\mathcal{F}}(\epsilon)-\int_{\tilde{M} \backslash C r(\tilde{h})} \delta_{\tilde{\mathcal{F}}}(\epsilon)
$$

The left side of the above identity is zero because by a straightforward computation

$$
W_{\mathcal{F}}(t, \epsilon)=n \chi(M) \operatorname{dim} \mathcal{W} \log \epsilon=\mathrm{W}_{\tilde{\mathcal{F}}}(\mathrm{t}, \epsilon) .
$$

On the right side of the identity, the term

$\int_{M \backslash C r(h)} \delta_{\tilde{\mathcal{F}}}(\epsilon)=\int_{M \backslash C r(h)}\left(\alpha_{\tilde{\mathcal{F}}}+(-1)^{n} \alpha_{\tilde{\mathcal{F}}_{\sharp}}\right)$ is zero because in view of Proposition 0.1 both $\int_{M \backslash C r(h)} \alpha_{\tilde{\mathcal{F}}}$ and $\int_{M \backslash C r(h)} \alpha_{\tilde{\mathcal{F}}^{\sharp}}(\epsilon)$ are zero for the trivial representation and a parallel Hermitian structure.

Consequently $\int_{M \backslash C r(h)} \delta_{\mathcal{F}}(0)=0$.

( D) Choose $\mu:=\mu_{0}$. Then the statement follows from formula 5.1 Proposition 0.1 and Proposition 3.2 (ii).

Proof of Theorem 0.1 Step 1: We first show that $\mathcal{S}(M, \rho)$ is of the form $\mathcal{S}(M, \rho)=F(\rho ; \Gamma) \cdot \chi(M)$. Note that when $\rho$ is isomorphic to $\rho^{\sharp}$, it follows, by using (B) for $n$ odd and (C) for $n$ even, that $\mathcal{S}(M, \rho)=0$. If $\chi(M)=0$, choose an even dimensional manifold $M^{\prime}$ with nonzero Euler Poincaré characteristic and with the same fundamental group as $M$ and choose the representation $\rho^{\prime}:=\rho^{\sharp}$. Since $\rho \otimes \rho^{\sharp}$ is isomorphic to its dual, and in view of (D)

$$
0=\mathcal{S}\left(M \times M^{\prime}, \rho \otimes \rho^{\sharp}\right)=\mathcal{S}(M, \rho) \cdot \chi\left(M^{\prime}\right) \operatorname{dim}(\mathcal{W}) .
$$

Hence the result is true if $\chi(M)=0$.

Suppose $\chi(M) \neq 0$. For any $M^{\prime}$ with the same fundamental group as $M$ and $\rho^{\prime}:=\rho^{\sharp}$, by the same argument as above for the case $\chi(M)=0$, one concludes from (D) that

$$
\mathcal{S}\left(M^{\prime}, \rho^{\sharp}\right)=-\mathcal{S}(M, \rho) \cdot \frac{\chi\left(M^{\prime}\right)}{\chi(M)} .
$$

This implies that for any closed manifold with fundamental group $\Gamma, \mathcal{S}(M, \rho)=$ $\chi(M) \cdot F(\rho ; \Gamma)$, where $F(\rho ; \Gamma)$ depends only on the representation $\rho$ up to an isomorphism. Notice that if $\rho$ is isomorphic to its dual $\rho^{\sharp}$, then by (B) and (C), $F(\rho ; \Gamma) \cdot \chi(M)=0$.

Step 2: It remains to show that $F(\rho ; \Gamma)=0$. Given $\Gamma$, choose a closed manifold $\left(M, x_{0}\right)$ with $\operatorname{dim} M=2 n$ and $\Gamma=\pi_{1}\left(M, x_{0}\right)$. Let $M_{1}:=M, M_{2}:=M \# \mathbb{C} P(n)$, $M_{3}:=\mathbb{C} P(n), M_{4}:=S^{2 n}$. Observe that $\pi_{1}\left(M_{2}, x_{0}\right)=\pi_{1}\left(M_{1}, x_{0}\right)=\Gamma$ and 
$\chi\left(M_{2}\right)=\chi\left(M_{1}\right)+(n-1)$. Let $\left(\mathcal{E}_{i} \rightarrow M_{i}, \nabla_{i}\right)$ be the following flat bundles: for $i=1,2$ the ones induced by the representation $\rho$ and for $i=3,4$ the flat bundles induced by $\varepsilon$, the unique representation on $\mathcal{W}$ of the group with one element. We show below that one can choose Hermitian structures, Riemannian metrics and generalized triangulations so that, by the locality of the density given in Proposition 0.1 (cf subsection 0.2, (P1),)

$$
\mathcal{S}(M \# \mathbb{C} P(n), \rho)-\mathcal{S}(M, \rho)=\mathcal{S}(\mathbb{C} P(n), \varepsilon)-\mathcal{S}\left(S^{2 n}, \varepsilon\right)
$$

On the other hand, by Step 1,

(5.4) $\mathcal{S}(M \# \mathbb{C} P(n), \rho)-\mathcal{S}(M, \rho)=F(\rho) \cdot(n-1) ; \mathcal{S}(\mathbb{C} P(n), \varepsilon)=0, \mathcal{S}\left(S^{2 n}, \varepsilon\right)=0$

and the claimed result follows.

To prove (5.3), decompose $M_{i}$ as a union of compact manifolds with boundary, $M_{i}=M_{i}(-) \cup M_{i}(+)$ so that

(a) $M_{i}(-) \cup M_{i}(+)=M_{i} ; \quad N_{i}:=M_{i}(-) \cap M_{i}(+) \sim S^{2 n-1} ;$

(b1) $M_{1}(-) \sim M_{2}(-) \sim M \backslash \operatorname{Int}\left(D^{2 n}\right)$;

(b2) $M_{1}(+) \sim M_{4}(+) \sim D^{2 n}$;

(b3) $M_{2}(+) \sim M_{3}(+) \sim \mathbb{C} P(n) \backslash \operatorname{Int}\left(D^{2 n}\right)$;

(b4) $M_{3}(-) \sim M_{4}(-) \sim D^{2 n}$.

Let $W_{i}(-)$ and $W_{i}(+)$ be the bordisms (cf [BFK2]),

$$
W_{i}(-):=\left(M_{i}(-), \emptyset, \partial\left(M_{i}(-)\right)\right) ; \quad W_{i}(+):=\left(M_{i}(+), \partial\left(M_{i}(+)\right), \emptyset\right) .
$$

The isomorphisms in (b1) - (b4) then become isomorphisms of bordisms.

Denote by $\left(\mathcal{E}_{i}(+), \nabla_{i}\right)$ respectively $\left(\mathcal{E}_{i}(-), \nabla_{i}\right)$ the restriction of the flat bundles $\mathcal{E}_{i}$ to $M_{i}(+)$, respectively to $M_{i}(-)$. Further we have the isomorphisms of flat bundles

(c1) $\left(\mathcal{E}_{1}(-), \nabla_{1}\right) \sim\left(\mathcal{E}_{2}(-), \nabla_{2}\right)$;

(c2) $\left(\mathcal{E}_{1}(+), \nabla_{1}\right) \sim\left(\mathcal{E}_{4}(+), \nabla_{4}\right)$;

(c3) $\left(\mathcal{E}_{2}(+), \nabla_{2}\right) \sim\left(\mathcal{E}_{3}(+), \nabla_{3}\right)$;

(c4) $\left(\mathcal{E}_{3}(-), \nabla_{3}\right) \sim\left(\mathcal{E}_{4}(-), \nabla_{4}\right)$.

Choose Riemannian metrics $g_{i}$ on $M_{i}$ which are product like near $N_{i}=$ $M_{i}(-) \cap M_{i}(+)$ and generalized triangulations $\tau_{i}=\left(h_{i}, g_{i}^{\prime}\right)$ with $g_{i}^{\prime}$ product like near $N_{i}, 0$ a regular value for $h_{i}$, and $h_{i}^{-1}(0)=N_{i}$. They induce generalized triangulations on $W_{i}(-)$ and $W_{i}(+)$. Choose Hermitian structures on $\mathcal{E}_{i}$, parallel near the critical points so that (c1)-(c4) are isomorphisms of Hermitian flat 
bundles. (This is always possible since the space of Hermitian structures which are parallel on a subset is contractible.) Using the locality of the density given in Proposition 0.1 (cf subsection 0.2, (P1)), (5.3) follows.

Remark to Theorem 0.1 Theorem 0.1 answers the question raised by several experts in the field, if the formula of Bismut-Zhang can be extended to $L_{2}$-torsion. Consider the system $(M, \mathcal{E}, \nabla, \mu, g, \tau)$ where $(M, g)$ is a closed Riemannian manifold, $(\mathcal{E}, \nabla)$ a finite dimensional flat bundle equipped with a Hermitian structure $\mu$ and $\tau$ a generalized triangulation of $M$. Suppose that $M$ is connected and let $x_{0} \in M$. Let $\Gamma$ be the fundamental group of $M, \Gamma=\pi_{1}\left(M, x_{0}\right)$, $\mathcal{N}$ the finite von Neumann algebra associated to $\Gamma, \rho$ the holonomy representation of $\Gamma$ on $\mathcal{E}_{x_{0}}$ and $\rho_{\text {reg }}$ the regular representation of $\Gamma$ on the $\mathcal{N}$-Hilbert module $l_{2}(\Gamma)$. Denote by $\rho^{\prime}$ the representation of $\Gamma$ on the $\mathcal{N}$-Hilbert module $l_{2}(\Gamma) \otimes_{\mathbb{C}} \mathcal{E}_{x_{0}}$ given by $\rho^{\prime}:=\rho_{\text {reg }} \otimes_{\mathbb{C}} \rho$, by $\mathcal{M}$ the flat bundle associated with the regular representation $\rho_{\text {reg }}$ and by $\mu_{0}$ the canonical parallel Hermitian structure on $\mathcal{M}$ induced from the scalar product on $l_{2}(\Gamma)$. The flat bundle induced by the representation $\rho^{\prime}$ is $\mathcal{M} \otimes_{\mathbb{C}} \mathcal{E}$ and is equipped with the Hermitian structure $\mu^{\prime}=\mu_{0} \otimes \mu$. Under the hypothesis of determinant class for the pair $\left(M, \rho^{\prime}\right)$ (conjecturally always satisfied,) one can use Lott's approach [L] to define the $L_{2}-$ analytic torsion of $(M, \mathcal{E}, \nabla, \mu, g)$ resp. the $L_{2}$-Reidemeister torsion of $(M, \mathcal{E}, \nabla, \mu, g, \tau)$. It is straightforward to check that these torsions are the analytic torsion of $\left(M, g, \rho^{\prime}, \mu^{\prime}\right)$ resp. the Reidemeister torsion of $\left(M, g, \rho^{\prime}, \mu^{\prime}, \tau\right)$ and that the local quantities expressing $\log \mathcal{R}$, cf Theorem 0.1, are the same for $\left(M, \rho^{\prime}, \mu^{\prime}, g, \tau\right)$ and $(M, \rho, \mu, g, \tau)$. Therefore, by (2.15), the quotient of the $L_{2}$-analytic and $L_{2}$-Reidemeister torsions is the same as the quotient of analytic and Reidemeister torsions .

\subsection{An invariant for odd dimensional manifolds.}

For $\Gamma$ a finitely presented group, and denote by $\operatorname{Rep}(\Gamma, \mathcal{W})$ the space of representations of $\Gamma$ on the $\mathcal{A}$-Hilbert module of finite type $\mathcal{W}$. This space has a structure of a complex analytic space of infinite dimension when $\mathcal{A}$ is of infinite dimension.

Let $\left(M, x_{0}\right)$ be a base pointed, closed, smooth odd dimensional manifold, with $\pi_{1}\left(M, x_{0}\right)=\Gamma$, and let $E$ be an Euler structure in the sense of Turaev cf [Tu]. Recall $\mathrm{cf}[\mathrm{B} 2],[\mathrm{Tu}]$ that the set of Euler structures on a base pointed connected manifold $\left(M, x_{0}\right)$ can be defined as the set of connected components of the space of continuous vector fields with the only zero at $x_{0}$ equipped with the compact open topology. Recall also that $H_{1}(M ; \mathbb{Z})$ acts on this set freely and transitively and because $\chi(M)=0$ there is a canonical identification between sets of Euler structures obtained by using different base points. By using the relative torsion we will associate a (real analytic) function $F(M, E): \operatorname{Rep}(\Gamma, \mathcal{W}) \rightarrow \mathbb{R}$ which is a smooth invariant for the triple $\left(M, x_{0}, E\right)$. We will calculate $F(M, E)$ 
for $M=S^{1} \times N, N$ is a closed simply connected even dimensional manifold and $E$ the canonical Euler structure, defined below. In this case $\pi_{1}\left(M, x_{0}\right)=\mathbb{Z}$ and the space $\operatorname{Rep}(\mathbb{Z}, \mathcal{W})$ can be identified with $G l_{\mathcal{A}}(\mathcal{W})$. Therefore we will denote a representation $\rho$ by $A \in G l_{\mathcal{A}}(\mathcal{W}), A=\rho(1)$.

\section{Definition of F(M,E):}

First note that an Euler class $E$ can be represented by the following data: (1) a generalized triangulation $\tau=\left(h, g^{\prime}\right)$,

(2) a spray, i.e a collection of disjoint smooth embeddings $\alpha_{x}:[0,1] \rightarrow M$ with $\alpha_{x}(0)=x_{0}, \alpha_{x}(1)=x, x \in C r(h)$. Let $K:=\cup_{x \in C r(h)} \alpha_{x}([0,1]) \subset M$ and refer to $K$ as the spray defined by the curves $\alpha_{x}$.

The data (1) and (2) provide a vector field $X$ by taking $X=-\operatorname{grad}_{g^{\prime}} h$ outside of a contractible smooth regular neighborhood $U$ of $K$ and extending $X$ inside $U$ with the only zero at $x_{0}$. This vector field determines an Euler structure and any Euler structure can be obtained in this way (cf [B2]).

For a representation $\rho \in \operatorname{Rep}(\Gamma, \mathcal{W})$ denote by $\mathcal{E}_{\rho}$ the flat bundle induced from $\rho$ and choose a Hermitian structure $\mu$ in $\mathcal{E}_{\rho}$, so that $\mu$ is parallel in the neighborhood of $x_{0}$ and $\mu\left(x_{0}\right)$ is exactly the scalar product of $\mathcal{W}\left(\mathcal{E}_{\rho}\left(x_{0}\right)=\mathcal{W}\right)$.

Define

$$
F(M, E)(\rho):=\log \mathcal{R}(M, \rho, \mu, \tau, g) .
$$

Using Propositions 4.1-4.3 one verifies that $F(M, E)(\rho)$ is well defined, i.e. is independent of the choice of triangulation, metric and Hermitian structure of [B2] Main Theorem (1).

Let $M=N \times S^{1}$ with $N$ simply connected and of even dimension.

Choose the generalized triangulation $\tau_{S^{1}}$ of $S^{1}=\mathbb{R} / \mathbb{Z}$ given by the canonical metric $g_{0}$ and a Morse function with two critical points, a minimum at $t_{1}$ and a maximum at $t_{2}$, and choose as a base point $t_{0}=1 / 2$. Suppose that $0<t_{1}<$ $1 / 2<t_{2}<1$. Take $K_{S^{1}}=\left[t_{1}, 1 / 2\right] \cup\left[1 / 2, t_{2}\right]$.

Choose a generalized triangulation $\tau_{N}$ of $N$, a base point $n_{0}$ and a spray $K_{N}$. Consider the Euler structure on $S^{1} \times N$ represented by $\tau_{S^{1}} \times \tau_{N}$ and spray $K=K_{S^{1}} \times K_{N}$ with base point $x_{0}=t_{0} \times n_{0}$. Denote by $E_{0}$ the Euler structure defined by these data. Since $N$ is simply connected this Euler structure is independent of the spray $K_{N}$ and the triangulation $\tau_{N}$ and will be refered as the canonical Euler structure.

The flat bundle induced by $A$ is actually the tensor product of a flat bundle induced by $A$ on $S^{1}$ for which we choose a Hermitian structure parallel above $\left[t_{1}-\epsilon, t_{2}+\epsilon\right]$, with the trivial line bundle on $N$, for which we choose a parallel Hermitian structure.

Proposition 5.1 For the canonical Euler structure $E_{N}$ described above $F\left(N \times S^{1}, E_{0}\right)(A)=-\chi(N) / 2 \log \operatorname{det}\left(A^{*} A\right)^{1 / 2}$.

Proof By the product formula for the relative torsion (use $\chi\left(S^{1}\right)=0$ )

$$
\log \mathcal{R}\left(N \times S^{1}, A, \mu, g, \tau\right)=\chi(N) \log \mathcal{R}\left(S^{1}, A, \mu_{S^{1}}, g_{0}, \tau_{S^{1}}\right) .
$$


We compute $\log \mathcal{R}\left(S^{1}, A, \cdots\right)$ using Theorem 0.1.

Note that $\mu_{S^{1}}$ can be viewed as a smooth family $\mu(t), t \in \mathbb{R}$ of scalar products in $\mathcal{W}$ with $\mu(t+1)=A^{-1^{*}} \mu(t) A^{-1}$ and $\mu(t)=\mu_{0}$ for $t \in\left[t_{1}+k-\right.$ $\left.\epsilon, t_{2}+k+\epsilon\right], k \in \mathbb{Z}$ and $\mu_{0}$ the scalar product of $\mathcal{W}$. Recall (subsection 4.1) that $\theta(\mu)(t)=d \alpha(t)$ where $\alpha(t)=\log \operatorname{vol}\left(i d:(\mathcal{W}, \mu(t)) \rightarrow\left(\mathcal{W}, \mu_{0}\right)\right)$ which in particular says that $\alpha(t)=0$ for $t \in\left[t_{1}-\epsilon, t_{2}+\epsilon\right]$. Clearly $\alpha(1)-\alpha(0)=$ $\log v o l A^{-1}$.

Further, $\Psi \in \Omega^{0}\left(T S^{1} \backslash S^{1}\right)$ (cf property $4.2^{i v}$ ) is the function given by $\Psi(t, \xi)=1 / 2(\forall \xi>0)$ and $\Psi(x, \xi)=-1 / 2(\forall \xi<0)$.

The vectorfield $X: S^{1} \backslash C r(h) \rightarrow T S^{1} \backslash S^{1}$ is given by $-\operatorname{grad}_{g^{\prime}} h$ and thus the pullback of $\Psi$ by $X$ is

$$
X^{*}(\Psi)(t)=1 / 2 \quad\left(\forall t \in[0,1] \backslash\left[t_{1}, t_{2}\right]\right)
$$

and

$$
X^{*}(\Psi)(t)=-1 / 2 \quad\left(\forall t \in\left(t_{1}, t_{2}\right)\right)
$$

Note that $X^{*}(\Psi)(t)=-1 / 2$ on the points where $\theta(\mu)$ is zero. Combining the above computations, one obtains

$$
\int_{S^{1} \backslash C r(h)} \theta(\mu) X^{*}(\Psi)=1 / 2 \int_{S^{1} \backslash C r(h)} \theta(\mu)=\frac{1}{2} \log \operatorname{vol}\left(A^{-1}\right) .
$$

By the formula given in Theorem 0.1, the claimed statement follows.

\section{Appendix A Lemma of Carey-Mathai-Mishchenko}

In this Appendix, we prove Lemma 1.14, using a deformation argument.

Introduce $f_{q}(t):=t f_{q}$ and $d_{q}(t):=\left(\begin{array}{cc}d_{1, q} & t f_{q} \\ 0 & d_{2, q}\end{array}\right)$ and obtain in this way a complex $\left(\mathcal{C}^{*}, d_{*}(t)\right)$.

By the same arguments as in the proof of Lemma 1.10 one concludes that $\left(\mathcal{C}^{*}, d_{*}(t)\right)$ is an algebraically acyclic, $\zeta$-regular complex of sF type.

Therefore, $\left(\mathcal{C}^{*}, d_{x}(t)\right)$ has a well defined torsion $T(t):=T\left(\mathcal{C}^{*}, d_{*}(t)\right)$, given by (cf $(1.21)$ )

$$
\log T(t)=\frac{1}{2} \sum(-1)^{q} \log \operatorname{det}\left(\underline{d}_{q}^{*}(t) \underline{d}_{q}(t)\right) .
$$

If $\frac{d}{d t} \log T(t)=0$, then

$$
\log T(\mathcal{C})=\log T(1)=\log T(0)=\log T\left(\mathcal{C}^{1}\right)+\log T\left(\mathcal{C}^{2}\right)
$$


where for the last equality we have used that $d_{q}(0)=\left(\begin{array}{cc}d_{1, q} & 0 \\ 0 & d_{2, q}\end{array}\right)$ and therefore $\Delta_{q}(0)=\left(\begin{array}{cc}\Delta_{1, q} & 0 \\ 0 & \Delta_{2, q}\end{array}\right)$.

The remaining part of this Appendix is devoted to the proof of the statement

$$
\frac{d}{d t} \log T(t)=0
$$

Introduce the Hodge decomposition of $\mathcal{C}^{j}, \mathcal{C}_{q}^{j}=\mathcal{C}_{q}^{j+} \oplus \mathcal{C}_{q}^{j-}(j=1,2)$. The differential $d_{j, q}$ then have the form

$$
d_{j, q}=\left(\begin{array}{cc}
0 & \underline{d}_{j, q} \\
0 & 0
\end{array}\right)
$$

Further, decompose $\mathcal{C}_{q}$,

$$
\text { (A.1) } \quad \mathcal{C}_{q}=\mathcal{C}_{q}^{1+} \oplus \mathcal{C}_{q}^{1-} \oplus \mathcal{C}_{q}^{2+} \oplus \mathcal{C}_{q}^{2-} \text {. }
$$

Then, $d_{q}(t)$ can be written as

$$
d_{q}(t)=\left(\begin{array}{cc|cc}
0 & \underline{d}_{1, q} & \alpha_{q} & \beta_{q} \\
0 & 0 & \varepsilon_{q} & \gamma_{q} \\
\hline 0 & 0 & 0 & \underline{d}_{2, q} \\
0 & 0 & 0 & 0
\end{array}\right)
$$

where $\alpha_{q} \equiv \alpha_{q}(t)=t \dot{\alpha}_{q}, \beta_{q}=t \dot{\beta}_{q}, \varepsilon_{q}=t \dot{\varepsilon}_{q}$ and $\gamma_{q}=t \dot{\gamma}_{q}$. From $d_{q+1}(t) d_{q}(t)=0$ we deduce

(A.2) $\quad \underline{d}_{1, q+1} \varepsilon_{q}=0 ; \varepsilon_{q+1} \underline{d}_{2, q}=0$

(A.3) $\quad \underline{d}_{1, q+1} \gamma_{q}+\alpha_{q+1} \underline{d}_{2, q}=0$.

As $\mathcal{C}^{1}$ and $\mathcal{C}^{2}$ are acyclic, $\underline{d}_{1, q}$ and $\underline{d}_{2, q}$ are isomorphisms, and (A.2) and (A.3) imply

(A.4) $\quad \varepsilon_{q}=0$

(A.5) $\quad \gamma_{q}=-\underline{d}_{1, q+1}^{-1} \alpha_{q+1} \underline{d}_{2, q}$.

Next, let us describe the Hodge decomposition of $\mathcal{C}_{*}=: \mathcal{C}_{*}^{+}(t) \oplus \mathcal{C}_{*}^{-}(t)$ of $\left(\mathcal{C}_{*}, d_{*}(t)\right)$.

\section{Lemma A.1}


(i) $\mathcal{C}_{q}^{+}=\operatorname{Kerd}_{q}=\left\{\left(x_{+}-\underline{d}_{1, q}^{-1} \alpha_{q} y_{+}, y_{+}, 0\right) \mid x_{+} \in \mathcal{C}_{q}^{1+} ; y_{+} \in \mathcal{C}_{q}^{2+}\right\}$

(ii) $\mathcal{C}_{q}^{-}=\operatorname{Ker}\left(d_{q-1}^{*}\right)=\left\{\left(0, x_{-},\left(\underline{d}_{1, q}^{-1} \alpha_{q}\right)^{*} x_{-}, y_{-}\right) \mid x_{-} \in \mathcal{C}_{q}^{1-} ; y_{-} \in \mathcal{C}_{q}^{2-}\right\}$.

Proof The statements follow from a straightforward verification.

We want to compute the $t$-derivative of $\log T(t)=\frac{1}{2} \sum(-1)^{q} \log \operatorname{det}\left(\underline{d}_{q}^{*}(t) \underline{d}_{q}(t)\right)$. Notice that

$$
\begin{aligned}
\frac{d}{d t}\left(d_{q}^{*}(t) d_{q}(t)\right) & =\left(\begin{array}{cc}
0 & 0 \\
f_{q}^{*} & 0
\end{array}\right)\left(\begin{array}{cc}
d_{1, q} & t f_{q} \\
0 & d_{2, q}
\end{array}\right)+\left(\begin{array}{cc}
d_{1, q}^{*} & 0 \\
t f_{q}^{*} & d_{2, q}^{*}
\end{array}\right)\left(\begin{array}{cc}
0 & f_{q} \\
0 & 0
\end{array}\right) \\
& =\left(\begin{array}{cc}
0 & 0 \\
f_{q}^{*} d_{1, q} & t f_{q}^{*} f_{q}
\end{array}\right)+\left(\begin{array}{ll}
0 & d_{1, q}^{*} f_{q} \\
0 & t f_{q}^{*} f_{q}
\end{array}\right) \\
& =\left(\begin{array}{cc}
0 & d_{1, q}^{*} f_{q} \\
f^{*} d_{1, q} & 2 t f_{q}^{*} f_{q}
\end{array}\right) .
\end{aligned}
$$

As $f$ is a morphism of trace class (cf Definition 1.10) we conclude that $\frac{d}{d t} d_{q}^{*} d_{q}(t)$ and therefore $\frac{d}{d t} \underline{d}_{q}^{*}(t) \underline{d}_{q}(t)$ are of trace class. In view of Proposition 1.3 and the fact that $\left(\underline{d}_{q}^{*}(t) \underline{d}_{q}(t)\right)^{-1}$ is bounded we deduce that $\frac{d}{d t}\left(\underline{d}_{q}^{*}(t) \underline{d}_{q}(t)\right)\left(\underline{d}_{q}^{*}(t) \underline{d}_{q}(t)\right)^{-1}$ is of trace class. Therefore

$$
\frac{d}{d t} \log \operatorname{det}\left(\underline{d}_{q}^{*}(t) \underline{d}_{q}(t)\right)=\operatorname{Tr}\left(\frac{d}{d t}\left(\underline{d}_{q}^{*}(t) \underline{d}_{q}(t)\right)\left(\underline{d}_{q}^{*}(t) \underline{d}_{q}(t)\right)^{-1}\right) .
$$

$$
\begin{aligned}
& \operatorname{Tr}\left(\frac{d}{d t}\left(\underline{d}_{q}^{*}(t) \underline{d}_{q}(t)\right)\left(\underline{d}_{q}^{*}(t) \underline{d}_{q}(t)\right)^{-1}\right)= \\
& =\operatorname{Tr}\left(\left(\frac{d}{d t} \underline{d}_{q}^{*}(t)\right) \underline{d}_{q}^{*}(t)^{-1}\right)+\operatorname{Tr}\left(\underline{d}_{q}(t)^{-1} \frac{d}{d t} \underline{d}_{q}(t)\right) \\
& =2 \operatorname{Re} \operatorname{Tr}\left(\left(\frac{d}{d t} \underline{d}_{q}(t)\right) \underline{d}_{q}(t)^{-1}\right)
\end{aligned}
$$

It is convenient to introduce $D_{q}:=d_{q}(t)$ and denote by $D_{q}^{-1}$ the operator given by $\left.D_{q}^{-1}\right|_{\mathcal{C}_{q+1}}=0$ and $\left.D_{q}^{-1}\right|_{\mathcal{C}_{q+1}^{+}}=\underline{d}_{q}(t)^{-1}$.

$$
\operatorname{Tr}\left(D_{q}^{-1} \frac{d}{d t} D_{q}\right)=\operatorname{Tr}\left(\underline{d}_{q}(t)^{-1} \frac{d}{d t} \underline{d}_{q}(t)\right) .
$$

With respect to the decomposition (A.1), $D_{q}^{-1}$ and $\dot{D}_{q} \equiv \frac{d}{d t} D_{q}\left(\dot{D}_{q}\right.$ is independent of $t$ ) take the form

$$
D_{q}^{-1}=\left(\begin{array}{cccc}
A_{11} & A_{12} & A_{13} & A_{14} \\
A_{21} & A_{22} & A_{23} & A_{24} \\
A_{31} & A_{32} & A_{33} & A_{34} \\
A_{41} & A_{42} & A_{43} & A_{44}
\end{array}\right) ; \dot{D}_{q}=\left(\begin{array}{cccc}
0 & 0 & \dot{\alpha}_{q} & \dot{\beta}_{q} \\
0 & 0 & 0 & \dot{\gamma}_{q} \\
0 & 0 & 0 & 0 \\
0 & 0 & 0 & 0
\end{array}\right) .
$$


Thus

$$
\dot{D}_{q} D_{q}^{-1}=\left(\begin{array}{cccc}
\dot{\alpha}_{q} A_{31}+\dot{\beta}_{q} A_{41} & * & * & * \\
* & \dot{\gamma}_{q} A_{42} & * & * \\
0 & 0 & 0 & 0 \\
0 & 0 & 0 & 0
\end{array}\right)
$$

and

(A.8) $\operatorname{Tr}\left(\dot{D}_{q} D_{q}^{-1}\right)=\operatorname{Tr}\left(\dot{\alpha}_{q} A_{31}+\dot{\beta}_{q} A_{41}\right)+\operatorname{Tr}\left(\dot{\gamma}_{q} A_{42}\right)$.

Lemma A.2 (i) $A_{41}=0$;

(ii) $\left(-A_{42} \underline{d}_{1, q+1}^{-1} \alpha_{q+1}+A_{43}\right)=\underline{d}_{2, q}^{-1}$;

(iii) $A_{42}=-A_{43}\left(\underline{d}_{1, q+1}^{-1} \alpha_{q+1}\right)^{*}$;

(iv) $\underline{d}_{2, q}^{-1}=A_{43}\left(\left(\underline{d}_{1, q+1}^{-1} \alpha_{q+1}\right)^{*}\left(\underline{d}_{1, q+1}^{-1} \alpha_{q+1}\right)+I d\right)$;

(v) $A_{42}=-\underline{d}_{2, q}^{-1}\left(I d+\left(\underline{d}_{1, q+1}^{-1} \alpha_{q+1}\right)^{*}\left(\underline{d}_{1, q+1}^{-1} \alpha_{q+1}\right)\right)^{-1}\left(\underline{d}_{1, q+1}^{-1} \alpha_{q+1}\right)^{*}$;

(vi) $\dot{\gamma}_{q} A_{42}=\underline{d}_{1, q+1}^{-1} \dot{\alpha}_{q+1}\left(I d+\left(\underline{d}_{1, q+1}^{-1} \alpha_{q+1}\right)^{*}\left(\underline{d}_{1, q+1}^{-1} \alpha_{q+1}\right)\right)^{-1}\left(\underline{d}_{1, q+1}^{-1} \alpha_{q+1}\right)^{*}$.

Proof (i) Take $x=\left(x_{+}, 0,0,0\right) \in \mathcal{C}_{q}$. By Lemma $A .1, x \in \mathcal{C}_{q+1}^{+}$. Then

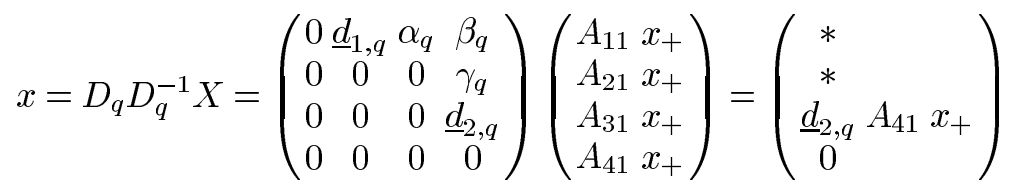

and therefore $0=\underline{d}_{2, q} A_{41} x_{+}$. As $\underline{d}_{2, q}$ is an isomorphism we conclude that $A_{41} x_{+}=0$.

(ii) Take $x\left(0,0,0, y_{-}\right) \in \mathcal{C}_{q}$. By Lemma A.1, $x \in \mathcal{C}_{q}^{-}$. Then

$$
\begin{aligned}
& x=D_{q}^{-1} D_{q} x=D_{q}^{-1}\left(\beta_{q} y_{-}, \gamma_{-}, \underline{d}_{2, q} y_{-}, 0\right)= \\
& =\left(*, *, *, A_{41} \beta_{q} y_{-}+A_{42} \gamma_{q} y_{-}+A_{43} \underline{d}_{2, q} y_{-}\right) .
\end{aligned}
$$

In view of (i), $A_{41}=0$ and thus

$$
\left(A_{42} \gamma_{q}+A_{43} \underline{d}_{2, q}\right) y_{-}=y_{-} .
$$

Recall that $\gamma_{q}=-\underline{d}_{1, q+1}^{-1} \alpha_{q+1} \underline{d}_{2, q}$ and substitute into (A.9) to obtain (ii). 
(iii) As $\left.D_{q}^{-1}\right|_{\mathcal{C}_{q+1}^{-}}=0$ and in view of Lemma A.1,

$$
\begin{aligned}
& 0=D_{q}^{-1}\left(0, x_{-},\left(\underline{d}_{1, q+1}^{-1} \alpha_{q+1}\right)^{*} x_{-}, 0\right)= \\
& =\left(*, *, *, A_{42} x_{-}+A_{43}\left(\underline{d}_{1, q+1}^{-1} \alpha_{q+1}\right)^{*} x_{-}\right) .
\end{aligned}
$$

Thus $A_{42}+A_{43}\left(\underline{d}_{1, q+1}^{-1} \alpha_{q+1}\right)^{*}=0$ which proves (iii)

(iv) follows from substituting (iii) into (ii).

(v) Notice that $I d+\left(\underline{d}_{1, q+1}^{-1} \alpha_{q+1}\right)^{*}\left(\underline{d}_{1, q+1}^{-1} \alpha_{q+1}\right) \geq I d$ is invertible and therefore, we can solve (iv) for $A_{43}$. Substituted into (iii) we obtain (v).

(vi) Substitute $\dot{\gamma}_{q}=-\underline{d}_{1, q+1}^{-1} \dot{\alpha}_{q+1} \underline{\dot{d}}_{2, q}$ into (v) to get (vi).

\section{Lemma A.3}

(i) $\alpha_{q} A_{31}+\underline{d}_{1, q} A_{21}=I d\left(\right.$ on $\left.\mathcal{C}_{1, q}^{+}\right)$,

(ii) $A_{31}=\left(\underline{d}_{1, q}^{-1} \alpha_{q}\right)^{*} A_{21}$;

(iii) $A_{21}=\left(I d+\left(\underline{d}_{1, q}^{-1} \alpha_{q}\right)\left(\underline{d}_{1, q}^{-1} \alpha_{q}\right)^{*}\right)^{-1} \underline{d}_{1, q}^{-1}$;

(iv) $\dot{\alpha}_{q} A_{31}=\dot{\alpha}_{q}\left(\underline{d}_{1, q}^{-1} \alpha_{q}\right)^{*}\left(I d+\left(\underline{d}_{1, q}^{-1} \alpha_{q}\right)\left(\underline{d}_{1, q}^{-1} \alpha_{q}\right)^{*}\right)^{-1} \underline{d}_{1, q}^{-1}$.

Proof The proof of Lemma A.3 is similar to the one of Lemma A.2.

Lemma A.4

(i) $\sum_{q}(-1)^{q} \operatorname{Tr}\left(\dot{D}_{q} D_{q}^{-1}\right)=0$;

(ii) $\frac{d}{d t} \log \operatorname{det} T(t)=0$.

Proof (i) Substituting Lemma A.2 (i) and (vi) as well as Lemma A.3 (iv) into (A.8) leads to

$$
\begin{aligned}
\operatorname{Tr}\left(\dot{D}_{q} D_{q}^{-1}\right) & =\operatorname{Tr}\left(\dot{\alpha}_{q} A_{31}\right)+0+\operatorname{Tr}\left(\dot{\gamma}_{q} A_{42}\right) \\
& =\operatorname{Tr}\left(\dot{\alpha}_{q}\left(\underline{d}_{1, q}^{-1} \alpha_{q}\right)^{*}\left(I d+\left(\underline{d}_{1, q}^{-1} \alpha_{q}\right)\left(\underline{d}_{1, q}^{-1} \alpha_{q}\right)^{*}\right)^{-1} \underline{d}_{1, q}^{-1}\right) \\
& +\operatorname{Tr}\left(\underline{d}_{1, q+1}^{-1} \dot{\alpha}_{q+1}\left(I d+\left(\underline{d}_{1, q+1}^{-1} \alpha_{q+1}\right)^{*}\left(\underline{d}_{1, q+1}^{-1} \alpha_{q+1}\right)\right)^{-1}\left(\underline{d}_{1, q+1}^{-1} \alpha_{q+1}\right)^{*}\right) \\
& =\operatorname{Tr}\left(\dot{\alpha}_{q}\left(\underline{d}_{1, q}^{-1} \alpha_{q}\right)^{*}\left(I d+\left(\underline{d}_{1, q}^{-1} \alpha_{q}\right)\left(\underline{d}_{1, q}^{-1} \alpha_{q}\right)^{*}\right)^{-1} \underline{d}_{1, q}^{-1}\right. \\
& +\operatorname{Tr}\left(\dot{\alpha}_{q+1}\left(\underline{d}_{1, q}^{-1} \alpha_{q}\right)^{*}\left(I d+\left(\underline{d}_{1, q+1}^{-1} \alpha_{q+1}\right)^{*}\left(\underline{d}_{1, q+1}^{-1} \alpha_{q+1}\right)\right)^{-1} \underline{d}_{1, q+1}^{-1}\right)
\end{aligned}
$$

where for the last equality we have used the fact that $\operatorname{Tr}(A B)=\operatorname{Tr}(B A)$ and $\left(I d+A A^{*}\right)^{-1} A^{*}=A^{*}\left(I d+A A^{*}\right)^{-1}$. Thus with $\eta_{q}:=\underline{d}_{1, q}^{-1} \alpha_{q}$

$$
\begin{aligned}
\sum_{q}(-1)^{q} \operatorname{Tr}\left(\dot{D}_{q} D_{q}^{-1}\right) & =\sum(-1)^{q} \operatorname{Tr}\left(\dot{\alpha}_{q} \eta_{q}^{*}\left(I d+\eta_{q} \eta_{q}^{*}\right)^{-1} \underline{d}_{1, q}^{-1}\right) \\
& -\sum(-1)^{q+1} \operatorname{Tr}\left(\dot{\alpha}_{q+1} \eta_{q+1}^{*}\left(I d+\eta_{q+1} \eta_{q+1}^{*}\right) \underline{d}_{1, q+1}^{-1}\right) \\
& =0 .
\end{aligned}
$$


(ii) follows from (i) and (A.6).

\section{Appendix B Determinant class property}

In this appendix, we discuss the concept of determinant class and provide examples of pairs $(M, \rho)$ which are not of determinant class.

Given an $\mathcal{A}$-Hilbert module $\mathcal{W}$ and $\varphi \in \mathcal{L}_{\mathcal{A}}(\mathcal{W})$ an operator which satisfies $O p 1-O p 6$, one says that $\varphi$ is of determinant class (cf [BFKM]) iff

(B.1) $\quad \int_{0+}^{1} \log \lambda d F_{|\varphi|}(\lambda)>-\infty$

where $|\varphi|=\left(\varphi^{*} \cdot \varphi\right)^{1 / 2}$.

It is not difficult to provide morphisms $\varphi$ which are not of determinant class. For example for $\mathcal{A}=\mathcal{N}(\mathbb{Z})$ and $\mathcal{W}=l_{2}(\mathbb{Z})=L^{2}\left(S^{1} ; \mathbb{C}\right), S^{1}=\mathbb{R} / \mathbb{Z}$, the multiplication by a function $\alpha \in L^{\infty}\left(S^{1} ; \mathbb{C}\right)$ defines an element $\varphi=M_{\alpha} \in$ $\mathcal{L}_{\mathcal{A}}(\mathcal{W})$ which satisfies $O p 1-O p 6$. Take $\alpha:[0,1) \rightarrow \mathbb{C}$ defined by $\alpha(x)=$ $\exp \left(-1 / x^{2}\right)$. As $F_{\left|M_{\alpha}\right|}(\lambda)=\mu(\{x \in[0,1],|\alpha(x)| \leq \lambda\})$, where $\mu(X)$ denotes the Lebesgue measure of the set $X$, one can see that $F_{\left|M_{\alpha}\right|}(\lambda)=-(\log \lambda)^{1 / 2}$ for $0<\lambda<1 / e$ and the integral (B.1) is not convergent.

Consider $(K, \tau, \rho, \mu)$ where $(K, \tau)$ is a CW complex with finitely many cells in each dimension, $\rho$ is a representation of the group $\pi=\pi_{1}(K)$ on the $\mathcal{A}$ Hilbert module of finite type $\mathcal{W}$, and $\mu$ is a Hermitian structure in the flat bundle $\mathcal{E} \rightarrow \mathcal{K}$ induced by $\rho$.

We say that $(K, \tau, \rho, \mu)$ is of c-determinant class (cf [BFKM]) iff the associated cochain complex of $\mathcal{A}$-Hilbert modules of finite type $C^{*}(K, \tau, \rho, \mu)$ is of determinant class, i.e. $\delta_{i}$, or equivalently the combinatorial Laplacians $\Delta_{i}^{c o m b}$, are of determinant class for all $i$.

We also say that $(M, g, \rho, \mu)$, with $(M, g)$ a smooth closed Riemannian manifold and $(\rho, \mu)$ as above, is of a- determinant class if the deRham complex $\Omega^{*}(M, \rho)$ of $\mathcal{A}$ - Hilbert modules whose Hilbert module structure is given by the scalar products induced by $g$ and $\mu$, is of determinant class, i.e. $d_{i}$, or equivalently the Laplacians $\Delta_{i}$, are of determinant class.

It was shown in [BFKM] (actually only for $\mu$ parallel, but the same arguments remain valid in the generality presented here) that the c-determinant class property is independent of $\tau$ and $\mu$, the a-determinant class property is independent of $g$ and $\mu$ and both properties are homotopy invariant. Moreover for a compact manifold, possibly with boundary, the a-determinant class property holds iff the c-determinant class property holds. Therefore the determinant class property is a homotopy invariant for a pair $(K, \rho)$ with $K$ a compact space of the homotopy type of a CW complex, which can be defined both analytically and combinatorially.

If $\rho$ is a representation of $\Gamma$, induced by a homomorphism $\pi: \Gamma \rightarrow G, G$ a countable discrete group, on the $\mathcal{N}(G)$ - Hilbert module $\mathcal{W}=l_{2}(\Gamma)$, then $(K, \rho)$ is of determinant class when $G$ is residually finite or $G$ is amenable. Recently 
B.Clair $[\mathrm{C}]$ has verified the determinant class property when $G$ is a residually amenable group.

A representation $\rho: \pi_{1}\left(S^{1}\right)=\mathbb{Z} \rightarrow \mathcal{L}_{\mathcal{N}(\mathbb{Z})}\left(L^{2}\left(S^{1} ; \mathbb{C}\right)\right)$ is determined by $\rho(1)$. Assume that $\rho(1)$ is the operator $\mathcal{M}_{1+\alpha}$ given by multiplication by $1+\alpha$ where $\alpha \in L^{\infty}\left(S^{1} ; \mathbb{C}\right)$. Consider the cochain complex induced by $\rho$ and the generalized triangulation $\tau=(h, g)$ with $h(t)=\frac{\cos (2 \pi t)+1}{2}$ and $g$ the standard metric. As the triangulation $\tau$ has one 0-cell $E_{0}$ and one 1-cell $E_{1}$, the cochain complex is of the form

$$
0 \rightarrow W \stackrel{\delta}{\rightarrow} W \rightarrow 0
$$

where $\delta\left(E_{0}\right)=E_{1}-\mathcal{M}_{1+\alpha} E_{1}=-\mathcal{M}_{\alpha} E_{1}$. Therefore,

$$
\log T_{\text {comb }}=\frac{1}{2} \log \operatorname{det} \delta^{*} \delta=\frac{1}{2} \log \operatorname{det} \alpha^{*} \alpha .
$$

Observe that $(M, \rho)$ is of determinant class iff $\mathcal{M}_{\alpha}$ is of determinant class, thus for $\alpha(x)=\exp \left(-1 / x^{2}\right)$, as introduced above, $\left(S^{1}, \rho\right)$ is NOT of determinant class. We point out that the regular representation of $\mathbb{Z}$ corresponds to the function $\alpha(t)=\exp (2 \pi i t)-1$. 


\section{Bibliography}

[B1] D.Burghelea: "Lectures on Witten- Helffer- Sjöstrand theory", E.S.I. preprint, Vienna, 1998.

[B2] D.Burghelea: "Removing Metric Anomalies from Ray- Singer Torsion", Letters in Mathematical Physics 47 (1999), p.149-158.

[BZ] J.P. Bismut, W. Zhang: "An extension of a theorem by Cheeger and Müller", Astérisque 205 (1992), p. 1-223.

[BFK1] D. Burghelea, L. Friedlander, T. Kappeler: "Witten deformation of the analytic torsion and the Reidemeister torsion", AMS Transl. (2), vol 184 (1998), p. 23-34

[BFK2] D. Burghelea, L. Friedlander, T. Kappeler: "Torsions for manifolds with boundary and gluing formulas", IHES preprint, 1996, (to appear in Math. Nach.).

[BFK3] D. Burghelea, L. Friedlander, T. Kappeler: "Asymptotic expansion of the Witten deformation of the analytic torsion", J. Funct. Anal. 137 (1996), p. 320-363.

[BFK4] D. Burghelea, L. Friedlander, T. Kappeler: "Mayer-Vietoris type formula for determinants of elliptic differential operators", J. Funct. Anal. 107 (1992), p. 34-66.

[BFK5] D. Burghelea, L. Friedlander, T. Kappeler: "Relative torsion for homotopy triangulation", to appear in Contemporary Mathematics, AMS 1999.

[BFKM] D. Burghelea, L. Friedlander, T. Kappeler, P. McDonald: "Analytic and Reidemeister torsion for representations in finite type Hilbert modules", GAFA 6 (1996), p. 751-859.

[C] B. Clair: "Residual amenability and the approximation of $L^{2}$ -invariants," preprint Univ. Chicago ,1997.

[CFM] A.L. Carey, M.Farber, V. Mathai: "Determinant lines, von Neumann algebras and $L^{2}$ torsion," J.reine angew. Math.484 (1997), p. 153181.

[CMM] A.L. Carey, V. Mathai, A. Mishchenko: "On analytic torsion over $C^{*}$-algebras", preprint.

[Di] J. Dixmier: $C^{*}$-algebras, North-Holland, Math. Library, Vol 15, 1997. 
[HS] B. Helffer, J. Sjöstrand: "Puits multiples en mécanique semi-classique IV - Etude du complexe de Witten", Comm. in PDE 10 (1985), p. 245340.

[Gi] P. Gilkey: "Invariance theory, the heat equation and the Atiyah-Singer index theorem", Publish or Perish, Wilmington, 1984.

[GS] M. Gromov, M.A. Shubin: "Von Neumann spectrum near zero", GAFA 1 (1991), p. 375-404.

[L] J.Lott: "Heat kernels on covering spaces and topological invariants, J. of Diff. Geo. 35 (1992), p 471-510.

[RS] M. Reed, B. Simon: "Methods in modern mathematical physics", Vol 1, $2^{\text {nd }}$ ed., Academic Press, New York, 1980.

[Tu] Turaev,V : "Euler structures, nonsingular vector fields and torsion of Reidemeister type", Izv.Acad. Sci. USSR. 53 (1989), p 130-146.

[Wi] E. Witten: "Super symmetry and Morse theory", J. of Diff. Geom. 17 (1982), p. 661-692. 